\title{
Investigation of solid/vapor interfaces using ambient pressure X-ray photoelectron spectroscopy
}

\author{
D.E. Starr ${ }^{1}$, Z. Liu ${ }^{2}$, M. Hävecker ${ }^{3,4}$, A. Knop-Gericke ${ }^{3}$, H. Bluhm ${ }^{5, *}$ \\ ${ }^{1}$ Solar Energy Research, Energy Materials In-situ Laboratory (EMIL), Helmholtz-Zentrum Berlin für \\ Materialien und Energie GmbH, D-12489 Berlin, Germany \\ ${ }^{2}$ Advanced Light Source, Lawrence Berkeley National Laboratory, Berkeley, CA 94720, USA. \\ ${ }^{3}$ Fritz Haber Institute of the Max Planck Society, Department of Inorganic Chemistry, 14195 Berlin, \\ Germany. \\ ${ }^{4}$ Catalysis for Energy, Group E-GKAT, Helmholtz-Zentrum Berlin für Materialien und Energie GmbH, \\ D-12489 Berlin, Germany \\ ${ }^{5}$ Chemical Sciences Division, Lawrence Berkeley National Laboratory, Berkeley, CA 94720, USA.
}

\begin{abstract}
Heterogeneous chemical reactions at vapor/solid interfaces play an important role in many processes in the environment and technology. Ambient pressure X-ray photoelectron spectroscopy (APXPS) is a valuable tool to investigate the elemental composition and chemical specificity of surfaces and adsorbates on the molecular scale at pressures of up to 130 mbar. In this review we summarize the historical development of APXPS since its introduction over forty years ago, discuss different approaches to minimize scattering of electrons by gas molecules, and give a comprehensive overview about the experimental systems (vapor/solid interfaces) that have been studied so far. We also present several examples for the application of APXPS to environmental science, heterogeneous catalysis, and electrochemistry.
\end{abstract}

Key Words: X-ray photoelectron spectroscopy, in situ studies, heterogeneous catalysis, electrochemistry

*Corresponding author. Email: hbluhm@lbl.gov. 


\section{Introduction}

The interfaces between gases and solids govern many processes in the environment, energy generation, and heterogeneous catalysis. Examples include the removal of harmful components from automotive exhaust streams ${ }^{1}$, the reaction of fuels and oxidizers at the electrodes of solid oxide fuel cells ${ }^{2}$, cloud droplet nucleation on atmospheric aerosols particles ${ }^{3}$, as well as the uptake and release of trace gases by polar snow packs ${ }^{4}$. There are a number of surface sensitive spectroscopies and microscopies that can be used to study vapor/solid interfaces, such as infrared spectroscopy (IR) ${ }^{5,6}$; vibrational sumfrequency generation $(\mathrm{VSFG})^{7,8} ; \mathrm{X}$-ray emission spectroscopy $(\mathrm{XES})^{9}$; surface X-ray diffraction $(\mathrm{SXRD}){ }^{10}$; scanning force microscopy $(\mathrm{SFM})$ in both contact $^{11}$ and non-contact ${ }^{12}$ modes; scanning tunneling microscopy $(\mathrm{STM})^{13}$; as well as transmission electron microscopy ${ }^{14}$ and scanning electron microscopy ${ }^{15}$.

X-ray photoelectron spectroscopy (XPS) is one of the most versatile methods for the investigation of surfaces on the atomic scale. ${ }^{16}$ It provides quantitative information about the elemental composition and chemical specificity (e.g., oxidation state) of the surface. Due to the strong interaction of electrons with atoms at typical electron energies used in XPS $(100 \mathrm{eV}-1000 \mathrm{eV})$, the mean free path of the electrons is only on the order of several monolayers, giving XPS exquisite surface sensitivity. ${ }^{17}$ However, photoelectrons are also strongly scattered by gas molecules, which complicates the application of XPS at elevated pressures. For instance, the inelastic mean free path of electrons with $100 \mathrm{eV}$ kinetic energy in 1 mbar water vapor is about $1 \mathrm{~mm}$, much shorter than the typical working distance between the sample and the entrance to the electrostatic lens system of an electron analyzer, which is a few centimeters. The attenuation of electrons by gas molecules can be overcome by differential pumping schemes; the most commonly used approaches are discussed in the next section. The use of differential pumping has led to the development of a variety of photoelectron spectrometers that can now operate at up to 130 mbar. This technique is known as ambient pressure or high pressure XPS to distinguish it from vacuum-based X-ray photoelectron spectroscopy. 
A timeline for the development of ambient pressure X-ray photoelectron spectroscopy (APXPS) is shown in Figure 1. Shortly after introducing vacuum-based XPS, Kai Siegbahn's group at Uppsala University built the first APXPS instruments in the early $1970 \mathrm{~s}^{18,19}$. These instruments featured several differential pumping stages between the sample compartment and the electrostatic lens system of a hemispherical analyzer and were mainly used for pioneering investigations of vapor/liquid interfaces. At the end of the 1970s, Joyner and Roberts developed an instrument with a similar differential pumping scheme for measurements of vapor/solid interfaces. ${ }^{20}$ Two of these systems were built, one located at Cardiff and the other in Novosibirsk in the mid-1980s. Shortly thereafter Grunze and collaborators developed an APXPS instrument which was installed at the University of Maine. ${ }^{21}$ All of the aforementioned systems used laboratory X-ray sources (anodes). The first synchrotron-based APXPS instrument was developed at Lawrence Berkeley National Laboratory (LBNL) at the end of the 1990s. This instrument featured a differentially-pumped electrostatic lens system, which increases the collection efficiency for electrons without sacrificing differential pumping performance (see next section). With few exceptions most of the instruments that were introduced over the last decade utilize some version of a differentially-pumped electrostatic lens system. The first instrument of this kind was installed at the Advanced Light Source (ALS), beamline 9.3.2 22 , and was followed by a second generation of instruments, developed jointly by the Fritz Haber Institute, Berlin, LBNL and Specs GmbH, Berlin, with one instrument installed at Berliner Elektronenspeicherring-Gesellschaft für Synchrotronstrahlung m.b.H. (BESSY II, Berlin) ${ }^{23}$ and a second at the ALS beamline 11.0.2 $2^{24}$. The use of synchrotron-based X-rays has many advantages (increased photon flux, smaller spot size, tunable photon energy) and thus there are now a number of APXPS instruments already operational $\left(\mathrm{ALS}^{22,24,25,26}\right.$, Bessy $^{23}, \mathrm{SSRL}^{27}, \mathrm{MAX}^{2} \mathrm{lab}^{28}$, NSLS, SLS, Photon Factory ${ }^{29}$ ), in commissioning (SOLEIL, ALBA) or under development (Shanghai, Diamond, SPring-8) at synchrotrons around the world. The proliferation of APXPS systems was greatly helped by the recent availability of commercial versions. ${ }^{30,31,32}$ With the improvement of small-spot, high flux, monochromatized laboratory X-rays sources a renaissance of laboratory-based APXPS instruments 
has begun several years ago ${ }^{33,34,35,36}$ (see Figure 1); this is likely where the strongest growth in this field will be in the future.

Figure 1 also shows the cumulative number of APXPS publications over time. The increase in the publication rate after the installation of the first synchrotron-based instruments reflects the wider user base that these instruments in general provide (as opposed to a lab-based instrument which is usually used by a single or just a few groups), but it also suggests that APXPS measurements are part of a larger trend in surface science, namely the increasing importance that investigations of surfaces under operating conditions have gained over the last decade. In this review we will give examples for APXPS investigations of vapor/solid interfaces in fields as diverse as environmental science, electrochemistry, and heterogeneous catalysis. We will start with a review of the basic design principles of APXPS instruments.

\section{Technical aspects}

In this section we will review the design principles for APXPS instruments on a general level. For a more detailed discussion the reader is pointed to recent review papers on the subject. . $^{37,38,39,40,41,42}$

\section{A) Differential pumping}

The principle obstacle to performing XPS experiments under elevated pressure conditions is scattering of electrons by gas molecules. Elastic scattering dominates at electron kinetic energies below $\sim 100 \mathrm{eV}$, while inelastic scattering is the main contribution to signal attenuation above $\sim 100 \mathrm{eV}$. The attenuation of the signal $I$ at pressure $p$ compared to the signal $I_{0}$ at pressure $p_{0}$ under vacuum conditions is proportional to exp- $(\sigma d p)$, with $d$ the distance that the electrons travel in a gas at pressure $p$, and $\sigma$ the scattering cross section, which depends on the chemical composition of the gas phase. Since the gas phase composition and thus the electron scattering cross section is a characteristic of a certain experiment, and the pressure in most cases is sought to be as high as possible, it follows that the distance, $d$, that the 
electrons travel through the gas phase needs to be limited to reduce loss of signal. Another requirement is to keep the electron detector and hemispherical analyzer under high vacuum $\left(<10^{-7}\right.$ mbar $)$. Since each differential pumping stage provides pressure differentials of about $10^{-2}$ to $10^{-5}$ (depending on aperture size, pumping speed, and type of gas), it follows that several differential pumping stages are needed if the sample is to be measured at pressures in the mbar range. In addition the X-ray source, be it an X-ray anode or a synchrotron, also needs to remain under high vacuum; therefore the X-rays are admitted to the in situ cell through an $\mathrm{X}$-ray transparent window, most commonly a silicon nitride or aluminum membrane (thickness $\sim 100 \mathrm{~nm}$ ), but differential pumping stages between the X-ray source and in situ cell have also been used.

The basic approach to all APXPS experiments, pioneered by Siegbahn et al. in their early designs, is the use of a differential pumping scheme, where the sample is located in an in situ measurement cell and is placed close to a differentially-pumped aperture. Since the pressure distribution in front of the aperture is not homogeneous and lower than the background pressure inside the in situ cell, the sample has to be placed at a distance of about two aperture diameters to ensure that the pressure drop across the aperture does not influence the heterogeneous reactions at the sample surface. From this consideration it follows that the size of the incident photon beam is the most important parameter for the determination of the pressure limit and signal strengths in APXPS experiments: A small incident photon spot allows a reduction of the aperture size (ideally matching the size of the photon spot on the sample), which in turn permits a smaller sample-aperture distance, thus reducing the path length of the electrons through the gas environment. A small entrance aperture to the differential pumping system also reduces the gas flow into the subsequent pumping stages and allows for larger secondary apertures with less detrimental effects on the electron collection efficiency.

Before we proceed to discuss various approaches to differential pumping in APXPS, a word is in order on the relative comparison of pressure limits in APXPS, of which there are two: 1) The threshold in situ chamber pressure for the pumping speed of the differential pumping system to cope with the gas flow, and 2) The threshold pressure for obtaining spectra with an acceptable signal-to-noise ratio at 
reasonable acquisition times. The answer to the second question is obviously the most important for APXPS measurements, and this pressure limit depends on a number of experimental parameters, in particular on the kinetic energy of the electrons (higher KE electrons are less scattered by the gas phase, but also provide less surface sensitivity), the type of gas or gas mixture in the experiment (e.g., the scattering cross sections for some selected gases increases in the order of $\mathrm{H}_{2}<\mathrm{He}<\mathrm{O}_{2}<\mathrm{CH}_{3} \mathrm{OH}$ ), the photoelectron emission cross section of the core level under investigation at the given photon energy, and the total flux as well as the beam size of the incident photon beam (the latter one determining the minimum distance between sample and aperture). All these parameters have a bearing on the detected signal, with each one of them easily changing the signal-to-noise levels by a factor of 10 or more. The question "What is the pressure limit in an APXPS experiment?" therefore requires a qualified answer which takes all of the above factors into account.

Figure 2 shows differential pumping schemes that have been developed for APXPS (please note that these are schematic representations, and may not resemble the real electron trajectories or relative dimensions). The most basic differential pumping system inserts two or more differential pumping stages between the sample location and the entrance to a standard electron energy analyzer input lens (see Figure 2a). The appeal of this scheme is its simplicity since it does not require any modifications to the electron optical components of a standard electron analyzer. On the other hand it requires a compromise between the differential pumping rates and the detection efficiency: Smaller apertures lead to larger pressure differentials but also reduce the acceptance angle of the electrons. Therefore, aperture shapes and sizes are often adjusted to fit the electron trajectories. This approach was used in the APXPS systems developed by Siegbahn et al. ${ }^{18,19}$, Joyner $\&$ Roberts ${ }^{20}$, Grunze et al. ${ }^{21}$, and Steinrück et al. ${ }^{34}$ All of these instruments use laboratory X-ray sources with spot sizes in the millimeter range, resulting in practical operating pressures of up to 1.3 mbar, which is a large step (indeed more than six orders of magnitude) in pressure towards more realistic operating conditions in XPS. A recently developed instrument by Nilsson et al. (SSRL) uses the same approach to differential pumping. ${ }^{27}$ In this case, however, the incident photon source is an undulator beamline, which provides a tightly focused, high flux photon source $(50 \mu \mathrm{m} \times 10$ 
$\mu \mathrm{m})$ and the use of a matching front aperture size of $50 \mu \mathrm{m}$ diameter. A reduction in the aperture diameter from $1 \mathrm{~mm}$ to $50 \mu \mathrm{m}$ reduces the gas flow into the electrostatic lens system by a factor of 400 (from a purely geometrical point of view) and therefore increases the pressure limit in terms of differential pumping by the same amount (all other parameters, such as pumping speed and conductance, being equal). It also allows the sample to be brought closer to the front aperture and thus reduces the attenuation of the signal by electron scattering with gas molecules. Using this instrument, Pt $4 \mathrm{f}$ spectra were obtained at pressures of up to 130 mbar of $\mathrm{O}_{2}$ using photoelectron kinetic energies of $\sim 930 \mathrm{eV}$ and an acquisition time of 1.5 hours.

To overcome the trade-off between differential pumping and efficiency of electron detection, a differential pumping system with integrated electrostatic lenses was introduced by Ogletree et al. in 2000 (see Figure $2 b$ ). ${ }^{22}$ A two stage differentially-pumped electrostatic lens transfers electrons from the sample plane onto the focal plane of a conventional hemispherical electron energy analyzer (Physical Electronics, Inc.). Since the exit aperture of the pre-lens is grounded, the effect of the pre-lens is to move the image plane farther away from the electron analyzer (in this case by $\sim 18 \mathrm{~cm}$ ) without changing the electron kinetic energies. Due to the large separation between the differentially-pumped apertures, the pumping speed in the differential stages is sufficient to provide a pressure differential of $10^{-8}$ between the in situ cell and the hemisphere, using apertures with diameters of $0.9 \mathrm{~mm}, 1.5 \mathrm{~mm}$ and $3.0 \mathrm{~mm}\left(1^{\text {st }}, 2^{\text {nd }}\right.$, and $3^{\text {rd }}$, respectively). Using electrostatic lenses in between the apertures, electrons are focused onto the aperture planes, thus mostly preserving the acceptance angle of the standard electrostatic lens while at the same time keeping the aperture sizes small and thus increasing differential pumping. This instrument allowed to record spectra at up to $7 \mathrm{mbar}$ of water vapor using $200 \mathrm{eV} \mathrm{KE}$ electrons. During the same period of time, Kelly et al. developed a two-stage differentially pumped system using electrostatic grid lenses, based on a laboratory X-ray source, with an upper pressure limit of $\sim 0.3$ mbar. $^{33}$ Most subsequently designed instruments have also employed differentially-pumped electrostatic lens stages.

The next generation of instruments, jointly developed by the Fritz Haber Institute in Berlin, LBNL, and Specs Surface Nano Analysis GmbH, Berlin, featured a modified electrostatic input lens (as 
opposed to a pre-lens; see Figure 2c). ${ }^{23,39}$ The front lens elements (of the intermediate image plane of the standard lens) of a Phoibos 150 hemispherical analyzer were replaced by two differential pumping/electrostatic lens stages, and the iris aperture (intermediate plane) replaced with a stationary aperture. Aperture sizes in this differentially pumped lens system are $0.9 \mathrm{~mm}, 2 \mathrm{~mm}$, and $2 \mathrm{~mm}\left(1^{\text {st }}, 2^{\text {nd }}\right.$, and $3^{\text {rd }}$, respectively), each separated by about $25 \mathrm{~cm}$, providing a pumping differential of $10^{-8}$ between the in situ cell and the hemisphere. These instruments are operating at BESSY II (ISISS beamline) and the ALS (beamline 11.0.2) and are also able to operate at water vapor pressures above 7 mbar for $200 \mathrm{eV}$ kinetic energy electrons. Fully commercial systems became available in about 2005 . While the Omicron analyzer uses scheme (a) ${ }^{34}$ in Figure 2, the APXPS spectrometers by Scienta and Specs use differentially pumped electrostatic pre-lenses in front of a standard input lens, following schemes $(d)^{25}$ and $(e)^{36}$, respectively.

\section{B) In situ chambers}

While the emphasis in the development of APXPS systems has until recently been mostly on improving differential pumping schemes, the design of precise sample environmental control (e.g., temperature, pressure, gas composition, irradiation with UV) has recently gained in importance. This is partly due to the ready availability of commercial spectrometers, but also due to the expansion of the user base for APXPS instruments to fields outside of surface science, where non-standard UHV environments are required. Most APXPS experiments can be classified by the type of sample preparation into one of the following three categories: 1) In situ sample preparation, i.e., sputtering, annealing, and thin film growth. These are mostly single or polycrystalline samples that can be regenerated in an attached preparation chamber or through heating in certain gases inside the in situ cell. 2) Ex-situ sample preparation, i.e., nanoparticles deposited onto a substrate, as well as powder catalysts. 3) Non-traditional samples, such as liquids, but also complex multicomponent devices (e.g., batteries, fuel cells).

The basic layout of in situ cells currently in use at APXPS instruments is shown in Figure 3. The most straightforward design is one where the in situ cell is also the vacuum chamber that separates the 
sample from the laboratory environment (Figure 3a). During an experiment the whole chamber is exposed to the gas atmosphere. In this layout the in situ cell is usually connected to a load lock and/or preparation chamber. The advantage of this design is its simplicity; possible disadvantages are crosscontamination between different experiments and the relatively large volume and internal wall areas. It is also difficult to quickly switch between UHV experiments and measurements at elevated pressures, since, once the chamber is exposed to mbar pressures of a gas, without a bake-out it usually takes several hours or days to return to UHV conditions (depending on the type of gas).

To overcome this obstacle, a different design uses in situ cells that are placed inside a vacuum chamber and sealed against it during exposure of the sample to gases (Figure 3b). The sample is transferred into the in situ cell using in-vacuum manipulators. ${ }^{28}$ This design enables to quickly switch between measurements at elevated pressures and UHV type experiments, and it also reduces the exposed chamber volume and wall area. It should be noted, however, that this is a more complex setup that requires precise manipulation of the sample for the safe transfer into and out of the in situ cell.

Another concept is the use of custom designed, tailor-made sample cells for specific applications (see Figure 3c). This is particularly advantageous for liquid samples, where cross-contamination and easy cleanup after an experiment are important considerations, as well as for complex sample environments, such as electrochemical cells, where often numerous electrical contacts (in addition to heater and thermocouple) have to be made in a secure way on a small scale for the simultaneous measurement of electrical properties during the APXPS investigations. ${ }^{43}$ This concept also allows the design of cells with minimal volume and wall area.

APXPS experiments will increasingly be coupled with other characterization methods, which provide simultaneous information about the sample and gas phase. Already now many investigations in heterogeneous catalysis combine APXPS with gas phase analysis using, e.g., mass spectrometers. In those experiments it is important to reduce the rate of dark conversion reactions through the choice of the correct chamber and sample stage materials, as well as infrared heating which selectively only raises the temperature of the sample. Similar considerations will be becoming increasingly more important in other 
fields of APXPS research, raising the demands for the correct design of the in situ cells and sample environments.

APXPS has been applied to investigate a wide range of samples, including metals, metal oxides, alkali halides, and liquids (not discussed in this review). As in vacuum-based XPS, sample charging of insulating samples poses a significant challenge, in particular since the use of flood guns is not possible under elevated pressure conditions. This problem is partially mitigated by the generation of electrons in the gas phase in the volume that is illuminated by the incident photon beam, which can reduce charging under favorable conditions. ${ }^{40}$ Homogeneous charging of the surface can be corrected by using the BE of a well-known core level as an intrinsic BE reference. The situation is more complicated for powder samples, where the heterogeneous morphology of the sample leads to inhomogeneous charging, which broadens photoelectron peaks and makes the analysis of core-level shifts virtually impossible. Due to the importance of powder samples in heterogeneous catalysis this is a pressing issue for APXPS. The design of differentially-pumped flood guns may be one strategy to overcome this problem.

\section{Examples for the application of APXPS to solid/vapor interfaces}

APXPS has been used to study a great variety of samples under a wide range of environmental conditions (e.g., temperature, pressure, gas composition, UV irradiation, electrical bias). In this review we will limit ourselves to the discussion of solid/vapor interfaces. Table 1 provides a list of peerreviewed reports of APXPS investigations of solids in the presence of gases at pressures above 0.001 mbar. 
Table 1: Published APXPS investigations of solid/vapor interfaces at pressure larger than $0.001 \mathrm{mbar}$, as of January 2013.

\begin{tabular}{|c|c|c|c|c|c|}
\hline Sample & Gases & $\begin{array}{c}\text { Max.Press. } \\
{[\mathrm{mbar}]}\end{array}$ & $\begin{array}{c}\text { Temp. } \\
{[\mathrm{K}]}\end{array}$ & Year & Ref. \\
\hline $\begin{array}{l}\text { Pd } \\
\text { - }(100) \\
\text { - }(100) \\
\text { - }(100) \\
\text { - nanoparticles/ } \mathrm{SiO}_{\mathrm{x}} \\
\text { - nanoparticles/ } \mathrm{SiO}_{\mathrm{x}} \\
\text { - }(111) \\
\text { - }(111) \\
\text { - }(111) \\
\text { - }(111) \\
\text { - }(111) \\
\text { - }(111) \\
\text { - }(111) \\
\text { - }(111) \\
\text { - }(111) \\
\text { - }(111) \\
\text { - }(111) \\
\text { - }(111) \\
\text { - particles/Ga } \mathrm{O}_{3} \\
\text { - PdZn near surface alloy } \\
\text { - polycrystalline foil } \\
\text { - polycrystalline foil } \\
\text { - polycrystalline foil } \\
\text { - polycrystalline foil } \\
\text { - particles on } \mathrm{C} \text { nanotubes } \\
\text { - } 5 \% \mathrm{Pd} \text { on } \mathrm{C} \text { nanotubes } \\
\text { - 5\% Pd/CeO } \mathrm{O}_{2} \\
\end{array}$ & $\begin{array}{c}\mathrm{CO}, \mathrm{O}_{2} \\
\mathrm{CO}, \mathrm{O}_{2} \\
\mathrm{CO}, \mathrm{O}_{2} \\
\mathrm{CO}, \mathrm{O}_{2} \\
\mathrm{CO}, \mathrm{O}_{2} \\
\mathrm{O}_{2} \\
\mathrm{H}_{2} \\
\text { trans-2-pentene, } \mathrm{H}_{2} \\
\mathrm{CO} \\
\mathrm{CO}, \mathrm{CH}_{3} \mathrm{OH} \\
\mathrm{O}_{2}, \mathrm{CH}_{3} \mathrm{OH} \\
\mathrm{O}_{2}, \mathrm{C}_{2} \mathrm{H}_{4} \\
\mathrm{O}_{2} \\
\mathrm{O}_{2} \\
\mathrm{H}_{2}, \text { pentyne, pentene } \\
\mathrm{CH}_{4}, \mathrm{O}_{2} \\
\mathrm{CO}, \mathrm{O}_{2} \\
\mathrm{H}_{2}, \mathrm{O}_{2} \\
\mathrm{CH}_{3} \mathrm{OH}, \mathrm{H}_{2} \mathrm{O} \\
\mathrm{H}_{2}, \mathrm{C}_{3} \mathrm{H}_{4} \\
\mathrm{H}_{2}, \text { alkynes, alkenes } \\
\mathrm{H}_{2}, \text { alkynes } \\
\mathrm{H}_{2}, \text { pentyne, pentene } \\
\mathrm{H}_{2}, \mathrm{C}_{3} \mathrm{H}_{4} \\
\mathrm{H}_{2}, \text { pentyne, pentene } \\
\mathrm{CO}_{2}, \mathrm{H}_{2}, \mathrm{O}_{2} \\
\end{array}$ & $\begin{array}{c}0.5 \\
0.1 \\
0.7 \\
0.5 \\
0.7 \\
1.0 \\
3.0 \\
0.7 \\
0.7 \\
0.7 \\
0.07 \\
0.002 \\
0.002 \\
0.4 \\
0.9 \\
0.33 \\
0.7 \\
0.25 \\
0.36 \\
1.1 \\
1.0 \\
7.5 \\
0.9 \\
1.1 \\
0.9 \\
0.5 \\
\end{array}$ & $\begin{array}{c}295-550 \\
300-640 \\
295-680 \\
295-550 \\
295-550 \\
295-900 \\
295-623 \\
295-523 \\
300 \\
200-400 \\
300-600 \\
330-923 \\
423-923 \\
430-872 \\
358-523 \\
420-875 \\
470-770 \\
448-723 \\
300-623 \\
353-393 \\
343-353 \\
343-353 \\
358-523 \\
353-393 \\
358-523 \\
293-523 \\
\end{array}$ & $\begin{array}{l}2011 \\
2012 \\
2013 \\
2011 \\
2012 \\
2005 \\
2005 \\
2005 \\
2005 \\
2003 \\
2012 \\
2006 \\
2006 \\
2006 \\
2006 \\
2007 \\
2012 \\
2012 \\
2010 \\
2010 \\
2008 \\
2008 \\
2006 \\
2010 \\
2006 \\
2006\end{array}$ & $\begin{array}{c}44 \\
45 \\
46 \\
44 \\
47 \\
48 \\
49 \\
50 \\
51 \\
52 \\
53 \\
54 \\
55 \\
56 \\
57 \\
58 \\
59 \\
60 \\
61 \\
62 \\
63,64 \\
65 \\
57 \\
62 \\
57 \\
66 \\
\end{array}$ \\
\hline $\begin{array}{l}\text { Pt } \\
-(557),(332) \\
-(533) \\
-(110) \\
-(111) \\
-(111) \\
-(111) \\
-(111)\end{array}$ & $\begin{array}{c}\mathrm{CO}, \mathrm{O}_{2} \\
\mathrm{NH}_{3}, \mathrm{NO}, \mathrm{O}_{2} \\
\mathrm{CO}, \mathrm{O}_{2} \\
\mathrm{O}_{2} \\
\mathrm{O}_{2}, \mathrm{NO}, \mathrm{NO}_{2} \\
\mathrm{NO} \\
\mathrm{C}_{2} \mathrm{H}_{4}\end{array}$ & $\begin{array}{c}0.5 \\
0.7 \\
0.5 \\
0.5 \\
0.25 \\
1.3 \\
1.3\end{array}$ & $\begin{array}{c}295 \\
295-770 \\
295-473 \\
295-620 \\
295-520 \\
295 \\
295\end{array}$ & $\begin{array}{l}2010 \\
2008 \\
2011 \\
2011 \\
2009 \\
2010 \\
2013\end{array}$ & $\begin{array}{c}67,68 \\
69 \\
70,71 \\
72 \\
73 \\
74 \\
75\end{array}$ \\
\hline
\end{tabular}




\begin{tabular}{|c|c|c|c|c|c|}
\hline $\begin{array}{l}\text { - Pt precipitated on } \mathrm{Mg}(\mathrm{Al}) \mathrm{O} \\
\text { - } \mathrm{Pt} \text { adatoms in } \mathrm{FeO}_{\mathrm{x}} / \mathrm{Pt}(111) \\
\text { - on } \mathrm{CeO}_{2} \\
\text { - } 5 \% \mathrm{Pt} / \mathrm{CeO}_{2} \\
\text { - nanoparticles } / \mathrm{SiO}_{2} / \mathrm{Si}(111) \\
\text { - nanoparticles on } \mathrm{GaN}\end{array}$ & $\begin{array}{c}\mathrm{H}_{2} \mathrm{O}, \mathrm{H}_{2}, \mathrm{C}_{2} \mathrm{H}_{6}, \mathrm{O}_{2} \\
\mathrm{CO} \\
\mathrm{O}_{2}, \mathrm{CO} \\
\mathrm{CO}, \mathrm{H}_{2}, \mathrm{O}_{2} \\
\mathrm{H}_{2}, \mathrm{O}_{2} \\
\mathrm{H}_{2}, \mathrm{O}_{2} \\
\end{array}$ & $\begin{array}{l}0.7 \\
1.0 \\
1.0 \\
0.5 \\
0.5 \\
0.5 \\
\end{array}$ & $\begin{array}{l}295-725 \\
295-510 \\
393-573 \\
293-523 \\
373-873 \\
293-800 \\
\end{array}$ & $\begin{array}{l}2007 \\
2011 \\
2007 \\
2006 \\
2012 \\
2012 \\
\end{array}$ & $\begin{array}{l}76 \\
77 \\
78 \\
79 \\
80 \\
81\end{array}$ \\
\hline $\begin{array}{l}\text { Au } \\
\text { - nanoparticles } / \mathrm{TiO}_{2}(110) \\
\text { - nanoparticles } / \mathrm{TiO}_{2} \text { powder } \\
\text { - nanoparticles on } \mathrm{SiO}_{2} \text { and } \mathrm{TiO}_{2} \\
\text { - nanoparticles on } \mathrm{SiO}_{2} \text { and } \mathrm{TiO}_{2}- \\
\text { - polycrystalline foil } \\
\text { - evaporated on } \mathrm{TiO}_{2}(110) \\
\text { - (111), (310), (533) }\end{array}$ & $\begin{array}{c}\mathrm{O}_{2}, \mathrm{CO} \\
\mathrm{O}_{2}, \mathrm{CO} \\
\mathrm{O}_{2}, \mathrm{CO} \\
\mathrm{O}_{2}, \mathrm{NO} \\
\mathrm{O}_{2}, \mathrm{CO} \\
\mathrm{O}_{2}, \mathrm{CO} \\
\mathrm{NO}\end{array}$ & $\begin{array}{c}1.0 \\
0.07 \\
0.2 \\
0.5 \\
1.0 \\
1.0 \\
0.005\end{array}$ & $\begin{array}{c}295 \\
295-350 \\
300-423 \\
300-473 \\
295 \\
295 \\
300-500\end{array}$ & $\begin{array}{l}2010 \\
2006 \\
2009 \\
2011 \\
2010 \\
2011 \\
2012 \\
\end{array}$ & $\begin{array}{l}82 \\
83 \\
84 \\
85 \\
82 \\
86 \\
87\end{array}$ \\
\hline $\begin{array}{l}\text { Ag } \\
\text { - polycrystalline foil } \\
\text { - polycrystalline foil } \\
\text { - polycrystalline foil } \\
\text { - foil, powder, (110), (111) } \\
\text { - nanoparticles on HOPG } \\
\text { - nanoparticles on } \mathrm{Si}\end{array}$ & $\begin{array}{c}\mathrm{C}_{2} \mathrm{H}_{4}, \mathrm{O}_{2} \\
\mathrm{O}_{2} \\
\mathrm{O}_{2} \\
\mathrm{O}_{2} \\
\mathrm{C}_{2} \mathrm{H}_{4}, \mathrm{O}_{2} \\
\mathrm{C}_{3} \mathrm{H}_{6}, \mathrm{O}_{2}\end{array}$ & $\begin{array}{c}0.7 \\
0.4 \\
0.01 \\
0.2 \\
0.4 \\
0.5\end{array}$ & $\begin{array}{c}295-520 \\
473 \\
300-700 \\
300-773 \\
423-483 \\
293-493 \\
\end{array}$ & $\begin{array}{l}2006 \\
1979 \\
1988 \\
2012 \\
2011 \\
2010 \\
\end{array}$ & $\begin{array}{l}88 \\
89 \\
90 \\
91 \\
92 \\
93\end{array}$ \\
\hline $\begin{array}{l}\mathbf{R h} \\
\text { - nanoparticles } \\
\text { - nanoparticles } / \mathrm{TiO}_{2} \\
\text { - (111) }\end{array}$ & $\begin{array}{c}\mathrm{CO}, \mathrm{O}_{2} \\
\mathrm{H}_{2}, \mathrm{O}_{2} \\
\mathrm{CO}, \mathrm{NO}\end{array}$ & $\begin{array}{l}0.5 \\
0.2 \\
0.7\end{array}$ & $\begin{array}{c}295-550 \\
573 \\
300-620\end{array}$ & $\begin{array}{l}2008 \\
2011 \\
2004 \\
\end{array}$ & $\begin{array}{l}94 \\
95 \\
96 \\
\end{array}$ \\
\hline $\begin{array}{l}\mathbf{B i} \\
-(0001)\end{array}$ & $\mathrm{O}_{2}$ & 0.1 & $145-290$ & 1981 & 97 \\
\hline $\begin{array}{l}\mathbf{R u} \\
-(0001) \\
-(0001) \\
-(0001) \\
-(0001) \\
-(10-10) \\
\text { - polycrystalline foil } \\
\text { - nanoparticles }\end{array}$ & $\begin{array}{c}\mathrm{CH}_{3} \mathrm{OH}, \mathrm{O}_{2} \\
\mathrm{CO}, \mathrm{O}_{2} \\
\mathrm{CO} \\
\mathrm{CH}_{3} \mathrm{OH}, \mathrm{O}_{2} \\
\mathrm{CH}_{3} \mathrm{OH}, \mathrm{O}_{2} \\
\mathrm{CH}_{3} \mathrm{OH}, \mathrm{O}_{2} \\
\mathrm{CO}, \mathrm{O}_{2} \\
\end{array}$ & $\begin{array}{l}0.1 \\
0.1 \\
0.5 \\
0.3 \\
0.3 \\
0.3 \\
0.3 \\
\end{array}$ & $\begin{array}{l}350-720 \\
350-600 \\
295-600 \\
320-620 \\
320-620 \\
320-620 \\
293-473 \\
\end{array}$ & $\begin{array}{l}2007 \\
2006 \\
2013 \\
2007 \\
2007 \\
2007 \\
2012 \\
\end{array}$ & $\begin{array}{c}98 \\
99 \\
100 \\
101 \\
101 \\
101 \\
102 \\
\end{array}$ \\
\hline $\begin{array}{l}\mathbf{V} \\
-8 \% \text { V/alumina } \\
\end{array}$ & n-butane, $\mathrm{O}_{2}$ & 0.9 & 723 & 2008 & 64 \\
\hline $\begin{array}{l}\text { Mo } \\
\text { - polycrystalline foil }\end{array}$ & $\mathrm{O}_{2}$ & 0.2 & $700-900$ & 1990 & 103 \\
\hline Ta & & & & & \\
\hline
\end{tabular}




\begin{tabular}{|c|c|c|c|c|c|}
\hline $\begin{array}{l}-\mathrm{Ta} / \mathrm{SiO}_{2} \\
-(100) \text { and polycrystalline }\end{array}$ & $\begin{array}{c}\mathrm{C}_{2} \mathrm{H}_{2}, \mathrm{O}_{2} \\
\mathrm{O}_{2} \\
\end{array}$ & $\begin{array}{c}0.04 \\
0.2 \\
\end{array}$ & $\begin{array}{l}293-920 \\
293-500 \\
\end{array}$ & $\begin{array}{l}2012 \\
2010 \\
\end{array}$ & $\begin{array}{l}104 \\
105 \\
\end{array}$ \\
\hline $\begin{array}{l}\mathbf{F e} \\
-\mathrm{Fe} / \mathrm{SiO}_{2} \\
\end{array}$ & $\mathrm{C}_{2} \mathrm{H}_{2}, \mathrm{O}_{2}$ & 0.04 & $293-920$ & 2012 & 104 \\
\hline $\begin{array}{l}\mathbf{N i} \\
\text { - nanoparticles on } \mathrm{CeO}_{2} \\
\text { - polycrystalline foil }\end{array}$ & $\begin{array}{c}\mathrm{H}_{2} \\
\mathrm{C}_{3} \mathrm{H}_{8}, \mathrm{O}_{2}\end{array}$ & $\begin{array}{l}1.3 \\
1.0 \\
\end{array}$ & $\begin{array}{c}293-773 \\
293-1000 \\
\end{array}$ & $\begin{array}{l}2010 \\
2013 \\
\end{array}$ & $\begin{array}{l}106 \\
107\end{array}$ \\
\hline $\begin{array}{l}\text { Co } \\
-(0001) \\
\text { - }(0001) \\
\text { - nanoparticles on carbon support } \\
\text { - nanoparticles }\end{array}$ & $\begin{array}{c}\mathrm{H}_{2}, \mathrm{O}_{2} \\
\mathrm{CH}_{3} \mathrm{OH}, \mathrm{O}_{2} \\
\mathrm{H}_{2}, \mathrm{O}_{2} \\
\mathrm{H}_{2}\end{array}$ & $\begin{array}{l}0.2 \\
0.3 \\
0.2 \\
0.1\end{array}$ & $\begin{array}{l}295-650 \\
520 \\
295-650 \\
295\end{array}$ & $\begin{array}{l}2011 \\
2010 \\
2011 \\
2011\end{array}$ & $\begin{array}{l}108 \\
109 \\
108 \\
110\end{array}$ \\
\hline $\begin{array}{l}\mathrm{Cu} \\
\text { - polycrystalline foil; } \mathrm{Zn} / \mathrm{Cu} \\
\text { - polycrystalline foil } \\
\text { - polycrystalline foil } \\
\text { - polycrystalline foil } \\
\text { - (110) } \\
\text { - (110) } \\
\text { - (110) } \\
\text { - (111) } \\
\text { - }(111) \\
\text { - on } \mathrm{ZnO} / \mathrm{Al}_{2} \mathrm{O}_{3} \\
\text { - on } \mathrm{ZnO}\end{array}$ & $\begin{array}{c}\mathrm{CO}_{2}, \mathrm{H}_{2} \mathrm{O} \\
\mathrm{N}_{2} \mathrm{H}_{4} \text { (hydrazine) } \\
\mathrm{CH}_{3} \mathrm{OH}, \mathrm{O}_{2} \\
\mathrm{CH}_{3} \mathrm{OH}, \mathrm{O}_{2} \\
\mathrm{H}_{2} \mathrm{O} \\
\mathrm{H}_{2} \mathrm{O} \\
\mathrm{CH}_{3} \mathrm{OH}, \mathrm{O}_{2} \\
\mathrm{H}_{2} \mathrm{O} \\
\mathrm{SO}_{2} \\
\mathrm{H}_{2} \\
\mathrm{H}_{2} \\
\end{array}$ & $\begin{array}{c}0.2 \\
0.01 \\
0.45 \\
0.06 \\
1.0 \\
1.0 \\
1.0 \\
1.0 \\
0.1 \\
0.25 \\
0.25 \\
\end{array}$ & $\begin{array}{c}295 \\
295-380 \\
295-725 \\
420-670 \\
275-520 \\
295 \\
320-770 \\
295 \\
173-473 \\
523 \\
298-523 \\
\end{array}$ & $\begin{array}{l}2008 \\
1986 \\
2004 \\
2003 \\
2008 \\
2007 \\
2006 \\
2007 \\
1988 \\
2012 \\
2008 \\
\end{array}$ & $\begin{array}{c}111 \\
112 \\
23 \\
113 \\
114 \\
115 \\
116 \\
117 \\
118 \\
119 \\
38 \\
\end{array}$ \\
\hline $\begin{array}{l}\mathrm{CuGaSe}_{2} \\
\text { - polycrystalline films }\end{array}$ & $\mathrm{O}_{2}, \mathrm{H}_{2} \mathrm{O}$ & 0.5 & $295-573$ & 2005 & 120 \\
\hline $\begin{array}{l}\text { Si } \\
- \text { wafer } \\
-(100) \\
\end{array}$ & $\begin{array}{c}\mathrm{O}_{2} \\
\mathrm{O}_{2}, \mathrm{H}_{2} \mathrm{O} \\
\end{array}$ & $\begin{array}{l}2 \times 10^{-4} \\
0.01-1\end{array}$ & $\begin{array}{l}295-775 \\
573-803 \\
\end{array}$ & $\begin{array}{l}2001 \\
2008 \\
\end{array}$ & $\begin{array}{c}121 \\
122,123 \\
\end{array}$ \\
\hline $\begin{array}{l}\text { C } \\
\text { - nanotubes } \\
\text { - nanotubes on } \mathrm{Ni} \\
\text { - nanotubes } \\
\text { - nanotubes } \\
\text { - nanodiamonds } \\
\text { - nanotubes } /(\mathrm{Au}, \mathrm{Pd}, \mathrm{Fe}, \mathrm{Ni}) / \mathrm{SiO}_{2} \\
\text { - nanotubes on } \mathrm{CoSi}{ }_{2} \\
\text { - nanotubes on } \mathrm{Ta}\end{array}$ & $\begin{array}{c}\mathrm{C}_{2} \mathrm{H}_{2}, \mathrm{NH}_{3} \\
\mathrm{Ar}, \mathrm{C}_{2} \mathrm{H}_{2}, \mathrm{H}_{2} \\
\mathrm{O}_{2} \\
\text { Butane, } \mathrm{O}_{2} \\
\mathrm{C}_{6} \mathrm{H}_{5} \mathrm{CH}_{2} \mathrm{CH}_{3} \\
\mathrm{C}_{2} \mathrm{H}_{2}, \mathrm{H}_{2}, \mathrm{O}_{2} \\
\mathrm{C}_{2} \mathrm{H}_{2} \\
\mathrm{C}_{2} \mathrm{H}_{2}\end{array}$ & $\begin{array}{c}0.1 \\
0.5 \\
0.5 \\
0.25 \\
0.25 \\
0.005 \\
0.2 \\
0.02 \\
\end{array}$ & $\begin{array}{c}293-925 \\
573-973 \\
293-723 \\
623-648 \\
293-723 \\
773 \\
873 \\
923 \\
\end{array}$ & $\begin{array}{l}2011 \\
2011 \\
2010 \\
2008 \\
2010 \\
2009 \\
2012 \\
2011 \\
\end{array}$ & $\begin{array}{c}124,125 \\
126 \\
127 \\
128 \\
129 \\
130 \\
131 \\
132 \\
\end{array}$ \\
\hline Alkane thiols & & & & & \\
\hline
\end{tabular}




\begin{tabular}{|c|c|c|c|c|c|}
\hline - self-assembled monolayer/ $\mathrm{Au}$ & $\mathrm{H}_{2} \mathrm{O}$ & 1.0 & 295 & 2008 & 133 \\
\hline $\begin{array}{l}\text { POPC lipids } \\
\text { - self-assembled monolayer/ } / \mathrm{SiO}_{2}\end{array}$ & $\mathrm{H}_{2} \mathrm{O}$ & 1.0 & 295 & 2008 & 133 \\
\hline $\begin{array}{l}\text { Glycine } \\
\text { - on } \mathrm{Cu}(110) \\
\text { - on } \mathrm{Pt}(111) \\
\end{array}$ & $\begin{array}{l}\mathrm{H}_{2} \mathrm{O} \\
\mathrm{H}_{2} \mathrm{O} \\
\end{array}$ & $\begin{array}{l}0.5 \\
0.3 \\
\end{array}$ & $\begin{array}{l}295-500 \\
300-550 \\
\end{array}$ & $\begin{array}{l}2011 \\
2012 \\
\end{array}$ & $\begin{array}{l}134 \\
135 \\
\end{array}$ \\
\hline $\begin{array}{l}\text { Alanine } \\
\text { - on } \mathrm{Cu}(110) \\
\end{array}$ & $\mathrm{H}_{2} \mathrm{O}$ & 0.5 & $295-500$ & 2011 & 134 \\
\hline $\begin{array}{l}\mathrm{TiO}_{2} \\
\text { - (110) (rutile) } \\
\text { - (110) (rutile) } \\
\text { - polycrystalline (anatase) } \\
\text { - (101) anatase }\end{array}$ & $\begin{array}{c}\mathrm{H}_{2} \mathrm{O} \\
\mathrm{NO}_{2}, \mathrm{H}_{2} \mathrm{O} \\
\mathrm{H}_{2} \mathrm{O} \\
\mathrm{NO}_{2}, \mathrm{H}_{2} \mathrm{O} \\
\end{array}$ & $\begin{array}{c}1.5 \\
10^{-6}-0.2 \\
0.8 \\
0.1\end{array}$ & $\begin{array}{c}265-800 \\
300 \\
295 \\
298 \\
\end{array}$ & $\begin{array}{l}2007 \\
2010 \\
2009 \\
2013 \\
\end{array}$ & $\begin{array}{l}136 \\
137 \\
138 \\
139 \\
\end{array}$ \\
\hline $\begin{array}{l}\mathrm{CuO}_{2} \\
\text { - on polycrystalline } \mathrm{Cu} \text { foil } \\
\text { - on } \mathrm{Cu}(111)\end{array}$ & $\begin{array}{l}\mathrm{H}_{2} \mathrm{O} \\
\mathrm{SO}_{2} \\
\end{array}$ & $\begin{array}{l}1.5 \\
0.1\end{array}$ & $\begin{array}{l}270-295 \\
173-673 \\
\end{array}$ & $\begin{array}{l}2008 \\
1988 \\
\end{array}$ & $\begin{array}{l}140 \\
118 \\
\end{array}$ \\
\hline $\begin{array}{l}\mathrm{CuO} \\
\text { - on } \mathrm{Cu}(111) \\
\text { - on polycrystalline foil } \\
\end{array}$ & $\begin{array}{c}\mathrm{SO}_{2} \\
\mathrm{~N}_{2} \mathrm{H}_{4} \text { (hydrazine) } \\
\end{array}$ & $\begin{array}{c}0.1 \\
0.01 \\
\end{array}$ & $\begin{array}{l}173-473 \\
295-380 \\
\end{array}$ & $\begin{array}{l}1988 \\
1986 \\
\end{array}$ & $\begin{array}{l}118 \\
112 \\
\end{array}$ \\
\hline $\begin{array}{l}\mathrm{CoO}_{\mathbf{x}} \\
-\mathrm{CoO} \text { on } \mathrm{Co}(0001) \\
-\mathrm{Co}_{3} \mathrm{O}_{4} \text { on } \mathrm{Co}(0001) \\
\end{array}$ & $\begin{array}{l}\mathrm{CH}_{3} \mathrm{OH}, \mathrm{O}_{2} \\
\mathrm{CH}_{3} \mathrm{OH}, \mathrm{O}_{2} \\
\end{array}$ & $\begin{array}{l}0.3 \\
0.3 \\
\end{array}$ & $\begin{array}{l}520 \\
520 \\
\end{array}$ & $\begin{array}{l}2010 \\
2010 \\
\end{array}$ & $\begin{array}{l}109 \\
109 \\
\end{array}$ \\
\hline $\begin{array}{l}\mathbf{W O}_{3} \\
\text { - in situ grown on Si wafer }\end{array}$ & $\mathrm{O}_{2}$ & 0.001 & 295 & 2001 & 121 \\
\hline $\begin{array}{l}\text { MgO } \\
-(100) \text { thin film on } \operatorname{Ag}(100)\end{array}$ & $\mathrm{H}_{2} \mathrm{O}$ & 0.5 & $263-573$ & 2011 & 141,142 \\
\hline $\begin{array}{l}\mathbf{I n}_{2} \mathbf{O}_{3} \\
\text { - deposited on glass } \\
\text { - ITO }\left(\mathrm{In}_{2} \mathrm{O}_{3}: \mathrm{SnO}_{2}=90: 10\right) \\
\end{array}$ & $\begin{array}{l}\mathrm{H}_{2}, \mathrm{O}_{2} \\
\mathrm{H}_{2}, \mathrm{O}_{2} \\
\end{array}$ & $\begin{array}{l}0.0005 \\
0.0005 \\
\end{array}$ & $\begin{array}{l}373-773 \\
373-773 \\
\end{array}$ & $\begin{array}{l}2006 \\
2006 \\
\end{array}$ & $\begin{array}{l}143 \\
143 \\
\end{array}$ \\
\hline $\begin{array}{l}(\mathbf{V O})_{2} \mathbf{P}_{2} \mathbf{O}_{7} \\
\text { - pressed pellets } \\
\text { - pressed pellets }\end{array}$ & $\begin{array}{c}n \text {-butane, } \mathrm{He}, \mathrm{O}_{2} \\
n \text {-butane, } \mathrm{O}_{2}\end{array}$ & $\begin{array}{l}1.5 \\
0.5 \\
\end{array}$ & $\begin{array}{l}423-673 \\
593-673 \\
\end{array}$ & $\begin{array}{l}2005 \\
2012 \\
\end{array}$ & $\begin{array}{c}144,145 \\
146\end{array}$ \\
\hline $\begin{array}{l}\mathbf{L a}_{\mathbf{x}} \mathbf{S r}_{1-\mathbf{x}} \mathbf{C o O}_{3-\delta} \\
-\mathrm{La}_{0.8} \mathrm{Sr}_{0.2} \mathrm{CoO}_{3-\delta}(100) \text { film } \\
-\mathrm{La}_{0.8} \mathrm{Sr}_{0.2} \mathrm{CoO}_{3-\delta} \text { pressed pellet } \\
-\mathrm{La}_{0.5} \mathrm{Sr}_{0.5} \mathrm{CoO}_{3-\delta}\end{array}$ & $\begin{array}{l}\mathrm{O}_{2} \\
\mathrm{O}_{2} \\
\mathrm{O}_{2} \\
\end{array}$ & $\begin{array}{l}0.7 \\
0.7 \\
0.2 \\
\end{array}$ & $\begin{array}{l}295-790 \\
295-790 \\
295-673 \\
\end{array}$ & $\begin{array}{l}2012 \\
2012 \\
2009 \\
\end{array}$ & $\begin{array}{l}147 \\
147 \\
148 \\
\end{array}$ \\
\hline $\begin{array}{l}\text { MoVTeNbO } \\
\text { - M1 phase } \\
\text { - M1 phase }\end{array}$ & $\begin{array}{l}\mathrm{C}_{3} \mathrm{H}_{8}, \mathrm{O}_{2}, \mathrm{H}_{2} \mathrm{O} \\
\mathrm{C}_{3} \mathrm{H}_{8}, \mathrm{O}_{2}, \mathrm{H}_{2} \mathrm{O}\end{array}$ & $\begin{array}{c}0.02 \\
0.3\end{array}$ & $\begin{array}{l}323-693 \\
293-623\end{array}$ & $\begin{array}{l}2012 \\
2010\end{array}$ & $\begin{array}{l}149 \\
150\end{array}$ \\
\hline
\end{tabular}




\begin{tabular}{|c|c|c|c|c|c|}
\hline $\begin{array}{l}\mathbf{L i}_{\mathbf{x}} \mathbf{V}_{\mathbf{2}} \mathbf{O}_{\mathbf{5}} \\
-\mathrm{Li}_{4+\mathrm{x}} \mathrm{Ti}_{5} \mathrm{O}_{12} / \mathrm{LiPON} / \mathrm{Li}_{\mathrm{x}} \mathrm{V}_{2} \mathrm{O}_{5} \text { cell }\end{array}$ & $\mathrm{O}_{2}$ & 0.66 & 298 & 2012 & 151 \\
\hline $\begin{array}{l}\mathrm{CeO}_{2} \\
\text { - (100), } \mathrm{Sm} \text { doped } \\
-5 \% \mathrm{Pd} / \mathrm{CeO}_{2} \\
\text { - } 5 \% \mathrm{Pt} / \mathrm{CeO}_{2} \\
\text { - with } \mathrm{Au}, \mathrm{Pt}, \mathrm{Pd} \mathrm{Cu} \text { nanoparticles }\end{array}$ & $\begin{array}{l}\mathrm{H}_{2}, \mathrm{H}_{2} \mathrm{O}, \mathrm{O}_{2} \\
\mathrm{CO}, \mathrm{H}_{2}, \mathrm{O}_{2} \\
\mathrm{CO}, \mathrm{H}_{2}, \mathrm{O}_{2} \\
\mathrm{CO} / \mathrm{H}_{2} \mathrm{O} / \mathrm{H}_{2}\end{array}$ & $\begin{array}{l}0.4 \\
0.5 \\
0.5 \\
3.0\end{array}$ & $\begin{array}{l}690-1000 \\
293-523 \\
293-523 \\
540\end{array}$ & $\begin{array}{l}2012 \\
2006 \\
2006 \\
2012 \\
\end{array}$ & $\begin{array}{r}152 \\
66 \\
79 \\
153 \\
\end{array}$ \\
\hline $\begin{array}{l}\mathrm{FeO}_{\mathbf{x}} \\
-\alpha-\mathrm{Fe}_{2} \mathrm{O}_{3}(0001) \\
-\alpha-\mathrm{Fe}_{2} \mathrm{O}_{3} \text { nanoparticles/} / \mathrm{SiO}_{2} \\
-\mathrm{FeO}_{\mathrm{x}} \text { film on } \mathrm{Pt}(111) \\
\text { - } \mathrm{FeO} \text { nanoparticles on } \mathrm{Au}(111) \\
-\alpha-\mathrm{Fe}_{2} \mathrm{O}_{3} \text { particle/film on } \mathrm{Au}(111) \\
-\mathrm{Fe}_{2} \mathrm{O}_{3} ; \mathrm{Fe}_{2} \mathrm{O}_{3} / \mathrm{Cu} ; \mathrm{Fe}_{2} \mathrm{O}_{3} / \mathrm{Cu} / \mathrm{K} / \mathrm{Si} \\
-\mathrm{Fe}_{3} \mathrm{O}_{4}(001)\end{array}$ & $\begin{array}{c}\mathrm{H}_{2} \mathrm{O} \\
\mathrm{O}_{2}, \mathrm{H}_{2}, \mathrm{CO} \\
\mathrm{CO} \\
\mathrm{H}_{2} \mathrm{O} \\
\mathrm{CO}, \mathrm{H}_{2} \mathrm{O} \\
\mathrm{CO}, \mathrm{H}_{2} \\
\mathrm{H}_{2} \mathrm{O}\end{array}$ & $\begin{array}{c}2.0 \\
0.3 \\
1.0 \\
10^{-8}-0.1 \\
0.2 \\
0.4 \\
1.0\end{array}$ & $\begin{array}{c}277-647 \\
295-673 \\
295-510 \\
295 \\
295-473 \\
293-623 \\
264-533 \\
\end{array}$ & $\begin{array}{l}2010 \\
2011 \\
2011 \\
2011 \\
2010 \\
2010 \\
2013\end{array}$ & $\begin{array}{c}154 \\
155 \\
77 \\
156 \\
157 \\
158 \\
159\end{array}$ \\
\hline $\begin{array}{l}\mathrm{SiO}_{2} \\
\text { - nanoparticles (unsupported) } \\
\text { - native oxide layer on } \mathrm{Si}(111)\end{array}$ & $\begin{array}{c}\mathrm{H}_{2} \mathrm{O}, \mathrm{N}_{2} \\
\mathrm{H}_{2} \mathrm{O}\end{array}$ & $\begin{array}{c}0.001 \\
4.0\end{array}$ & $\begin{array}{c}295 \\
263-294\end{array}$ & $\begin{array}{l}2010 \\
2007\end{array}$ & $\begin{array}{l}160 \\
161\end{array}$ \\
\hline $\begin{array}{l}\mathbf{G e O}_{2} \\
\text { - grown on } \mathrm{Ge}(100)\end{array}$ & $\mathrm{H}_{2} \mathrm{O}$ & 1.0 & $263-294$ & 2012 & 162 \\
\hline $\begin{array}{l}\mathrm{Al}_{2} \mathbf{O}_{3} \\
\text { - native oxide layer on } \mathrm{Al} \text { foil }\end{array}$ & $\mathrm{H}_{2} \mathrm{O}$ & 1.5 & $260-295$ & 2008 & 140 \\
\hline $\begin{array}{l}\text { KI } \\
-(100) \\
-(100) \\
-(100)\end{array}$ & $\begin{array}{c}\text { Butanol, } \mathrm{H}_{2} \mathrm{O} \\
\text { Ozone, } \mathrm{H}_{2} \mathrm{O} \\
\text { Ozone, } \mathrm{H}_{2} \mathrm{O}\end{array}$ & $\begin{array}{c}1.6 \\
35 \% \mathrm{RH} \\
65 \% \mathrm{RH} \\
\end{array}$ & $\begin{array}{c}261-295 \\
295 \\
295\end{array}$ & $\begin{array}{l}2007 \\
2008 \\
2010 \\
\end{array}$ & $\begin{array}{l}163 \\
164 \\
165 \\
\end{array}$ \\
\hline $\begin{array}{l}\mathbf{K B r} \\
-(100) \\
\text { - thin film on } \mathrm{SiO}_{2}\end{array}$ & $\begin{array}{l}\mathrm{H}_{2} \mathrm{O} \\
\mathrm{H}_{2} \mathrm{O} \\
\end{array}$ & $\begin{array}{l}1.5 \\
1.5 \\
\end{array}$ & $\begin{array}{l}263-295 \\
258-295 \\
\end{array}$ & $\begin{array}{l}2005 \\
2010\end{array}$ & $\begin{array}{l}166 \\
167 \\
\end{array}$ \\
\hline $\begin{array}{l}\mathrm{NaCl} \\
\text { - frozen solutions } \\
-(100) \\
-(100) \\
-(100), \text { with } \mathrm{Br} / \mathrm{Cl}=0.001-0.1\end{array}$ & $\begin{array}{l}\mathrm{H}_{2} \mathrm{O} \\
\mathrm{H}_{2} \mathrm{O} \\
\mathrm{H}_{2} \mathrm{O} \\
\mathrm{H}_{2} \mathrm{O}\end{array}$ & $\begin{array}{l}2.3 \\
2.0 \\
1.6 \\
1.6\end{array}$ & $\begin{array}{l}242-286 \\
261-308 \\
263-295 \\
261-295\end{array}$ & $\begin{array}{l}2010 \\
2008 \\
2009 \\
2008\end{array}$ & $\begin{array}{l}168 \\
169 \\
170 \\
171\end{array}$ \\
\hline $\begin{array}{l}\mathrm{NaClO}_{4} \\
\text { - pressed pellets }\end{array}$ & $\mathrm{H}_{2} \mathrm{O}$ & 1.7 & $264-295$ & 2009 & 170 \\
\hline $\begin{array}{l}\mathbf{B a F}_{2} \\
-(111)\end{array}$ & $\mathrm{H}_{2} \mathrm{O}$ & 1.5 & $259-300$ & 2012 & 172 \\
\hline $\mathrm{RbCl}$ & & & & & \\
\hline
\end{tabular}




\begin{tabular}{|c|c|c|c|c|c|}
\hline$-(100)$ & $\mathrm{H}_{2} \mathrm{O}$ & 1.6 & $261-295$ & 2012 & 173 \\
\hline $\begin{array}{l}\text { RbBr } \\
-(100) \\
\end{array}$ & $\mathrm{H}_{2} \mathrm{O}$ & 1.6 & 261-295 & 2012 & 173 \\
\hline $\begin{array}{l}\text { Ice }\left(\mathrm{H}_{2} \mathrm{O}\right) \\
\text { - polycrystalline } \\
\text { - polycrystalline } \\
\text { - polycrystalline } \\
\text { - polycrystalline }\end{array}$ & $\begin{array}{c}\mathrm{H}_{2} \mathrm{O} \\
\mathrm{NO}_{2}, \mathrm{H}_{2} \mathrm{O} \\
\text { acetone, } \mathrm{H}_{2} \mathrm{O} \\
\text { acetic acid, } \mathrm{H}_{2} \mathrm{O}\end{array}$ & $\begin{array}{l}3.9 \\
0.2 \\
0.3 \\
1.0\end{array}$ & $\begin{array}{c}234-271 \\
230 \\
218-243 \\
230-240\end{array}$ & $\begin{array}{l}2002 \\
2010 \\
2011 \\
2013\end{array}$ & $\begin{array}{l}174 \\
175 \\
176 \\
177\end{array}$ \\
\hline $\begin{array}{l}\text { Electrochemical cells } \\
-\mathrm{CeO}_{2-\mathrm{x}} / \mathrm{Au} / \mathrm{YSZ} / \mathrm{Pt} \\
-\mathrm{CeO}_{2-\mathrm{x}} / \mathrm{Au} / \mathrm{YSZ} / \mathrm{Pt} \\
-\mathrm{CeO}_{2-\mathrm{x}} / \mathrm{Au} / \mathrm{YSZ} / \mathrm{Pt} \\
-\mathrm{Ni} / \mathrm{Pt} / \mathrm{YSZ} \\
-\mathrm{Pt} / \mathrm{YSZ} \\
-\mathrm{Ni} / \mathrm{GDC} / \mathrm{YSZ} / \mathrm{Pt}\end{array}$ & $\begin{array}{c}\mathrm{H}_{2}, \mathrm{H}_{2} \mathrm{O} \\
\mathrm{H}_{2}, \mathrm{H}_{2} \mathrm{O} \\
\mathrm{H}_{2}, \mathrm{H}_{2} \mathrm{O} \\
\mathrm{H}_{2}, \mathrm{H}_{2} \mathrm{O} \\
\mathrm{H}_{2}, \mathrm{H}_{2} \mathrm{O} \\
\mathrm{CH}_{4}, \mathrm{H}_{2}, \mathrm{O}_{2}\end{array}$ & $\begin{array}{c}1.0 \\
1.0 \\
0.6 \\
0.25 \\
0.25 \\
0.2 \\
\end{array}$ & $\begin{array}{c}875-1025 \\
875-1025 \\
973-1023 \\
975 \\
825-1025 \\
973 \\
\end{array}$ & $\begin{array}{l}2010 \\
2009 \\
2012 \\
2010 \\
2012 \\
2013 \\
\end{array}$ & $\begin{array}{c}178,179, \\
180 \\
181 \\
43,182, \\
183 \\
184 \\
\end{array}$ \\
\hline $\begin{array}{l}\text { Phtalocyanines } \\
\text { - } \mathrm{CoPc}, \mathrm{FePc}\end{array}$ & $\mathrm{H}_{2}, \mathrm{O}_{2}$ & 0.4 & 293 & 2011 & 185 \\
\hline 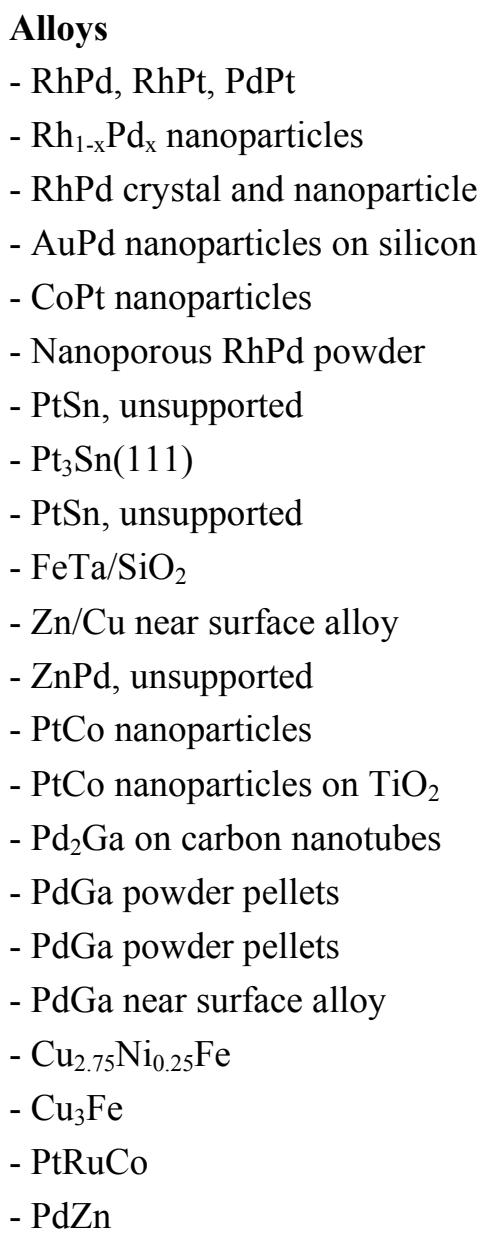 & $\begin{array}{c}\mathrm{O}_{2}, \mathrm{NO}, \mathrm{CO}, \mathrm{H}_{2} \\
\mathrm{CO}, \mathrm{O}_{2} \\
\mathrm{O}_{2}, \mathrm{NO}, \mathrm{CO} \\
\mathrm{O}_{2}, \mathrm{CO} \\
\mathrm{O}_{2}, \mathrm{CO}, \mathrm{H}_{2} \\
\mathrm{H}_{2}, \mathrm{O}_{2} \\
\mathrm{H}_{2}, \mathrm{O}_{2} \\
\mathrm{CO}, \mathrm{O}_{2} \\
\mathrm{O}_{2}, \mathrm{H}_{2}, \mathrm{C}_{6} \mathrm{H}_{12} \\
\mathrm{C}_{2} \mathrm{H}_{2}, \mathrm{O}_{2} \\
\mathrm{CH}_{3} \mathrm{OH}, \mathrm{H}_{2} \mathrm{O} \\
\mathrm{CH}_{3} \mathrm{OH}, \mathrm{H}_{2} \mathrm{O} \\
\mathrm{H}_{2}, \mathrm{O}_{2} \\
\mathrm{CH}_{3} \mathrm{OH}_{2} \mathrm{H}_{2} \mathrm{O}, \mathrm{H}_{2}, \mathrm{O}_{2} \\
\mathrm{H}_{2}, \mathrm{C}_{2} \mathrm{H}_{2} \\
\mathrm{H}_{2}, \mathrm{C}_{2} \mathrm{H}_{2} \\
\mathrm{H}_{2}, \mathrm{C}_{2} \mathrm{H}_{2} \\
\mathrm{CH}_{3} \mathrm{OH}_{2} \mathrm{H}_{2} \mathrm{O}, \mathrm{O}_{2} \\
\mathrm{O}_{2}, \mathrm{C}_{3} \mathrm{H}_{4}, \mathrm{H}_{2} \\
\mathrm{O}_{2}, \mathrm{C}_{3} \mathrm{H}_{4}, \mathrm{H}_{2} \\
\mathrm{CH}_{3} \mathrm{OH}, \mathrm{H}_{2} \mathrm{O}, \mathrm{CO}, \mathrm{O}_{2}, \\
\mathrm{H}_{2}\end{array}$ & $\begin{array}{c}0.2 \\
0.2 \\
0.2 \\
0.3 \\
1.0 \\
0.3 \\
0.6 \\
0.7 \\
0.5 \\
0.04 \\
0.35 \\
0.15 \\
0.2 \\
0.3 \\
1.1 \\
1.1 \\
1.1 \\
0.3 \\
1.0 \\
1.0 \\
0.5 \\
0.3\end{array}$ & $\begin{array}{c}573 \\
298-518 \\
295-623 \\
298-473 \\
300-400 \\
423 \\
393-573 \\
300-573 \\
573 \\
293-910 \\
300-693 \\
293-773 \\
450-520 \\
520-620 \\
393 \\
400 \\
400 \\
298-573 \\
523-783 \\
523-783 \\
570 \\
293-543\end{array}$ & $\begin{array}{l}2010 \\
2011 \\
2010 \\
2011 \\
2012 \\
2012 \\
2012 \\
2012 \\
2011 \\
2012 \\
2012 \\
2012 \\
2011 \\
2012 \\
2011 \\
2009 \\
2007 \\
2012 \\
2011 \\
2011 \\
2010 \\
2010\end{array}$ & $\begin{array}{c}186,187 \\
188 \\
189 \\
190 \\
191 \\
192 \\
193 \\
194 \\
195 \\
104 \\
196 \\
197 \\
198 \\
199 \\
200 \\
201 \\
202 \\
203 \\
204 \\
204 \\
205 \\
206\end{array}$ \\
\hline
\end{tabular}




\begin{tabular}{|l|c|c|c|c|c|}
\hline - PdZn & $\mathrm{CH}_{3} \mathrm{OH}, \mathrm{H}_{2} \mathrm{O}, \mathrm{CO}, \mathrm{O}_{2}$ & 0.5 & $293-523$ & 2012 & 207 \\
- PdZn near surface alloy & $\mathrm{CH}_{3} \mathrm{OH}, \mathrm{H}_{2} \mathrm{O}$ & 0.4 & $293-673$ & 2010 & 208 \\
- PdIn near surface alloy & $\mathrm{CH}_{3} \mathrm{OH}, \mathrm{H}_{2} \mathrm{O}$ & 0.2 & $298-673$ & 2012 & 209 \\
- $\mathrm{AgCu}$ & $\mathrm{O}_{2}, \mathrm{C}_{2} \mathrm{H}_{4}$ & 0.5 & 520 & 2010 & 210 \\
$-\mathrm{Co}_{0.5} \mathrm{Pt}_{0.5}$ nanoparticles & $\mathrm{H}_{2}$ & 0.1 & 293 & 2011 & 110 \\
$-\mathrm{Co}_{0.5} \mathrm{Pt}_{0.5}$ nanoparticles & $\mathrm{CO}, \mathrm{O}_{2}$ & 1.0 & $293-418$ & 2012 & 211 \\
- $\mathrm{RhPd}$ bilayers on $\mathrm{SiO}_{2}$ & $\mathrm{CO}, \mathrm{NO}$ & 0.03 & $293-670$ & 2012 & 212 \\
- PtAg on YSZ & $\mathrm{O}_{2}, \mathrm{C}_{2} \mathrm{H}_{4}$ & 0.25 & 650 & 2012 & 213 \\
- ZnNi, unsupported powder & $\mathrm{CH}_{3} \mathrm{OH}, \mathrm{H}_{2} \mathrm{O}$ & 0.2 & $298-693$ & 2012 & 214 \\
$-\mathrm{RuCoO}_{\mathrm{x}}$ & $\mathrm{CO}_{2}, \mathrm{H}_{2}$ & 0.7 & $373-773$ & 2012 & 215 \\
\hline
\end{tabular}

In the following we present examples of APXPS experiments on solid/vapor interfaces, from highly-ordered single crystals to supported nanoparticles and multicomponent model solid oxide fuel cell devices.

\section{A) AP-XPS experiments of adsorbates on single crystal surfaces}

Surface science experiments on single crystal surfaces under ultra-high vacuum conditions have a long history of providing detailed molecular and atomic level information about adsorbate-surface interactions. For APXPS experiments on single crystal surfaces this level of detail can still be achieved, but in addition the elevated pressure conditions extend the thermodynamic phase-space that can be explored with photoelectron spectroscopy. The advantage of using single crystal substrates stems directly from their well-defined, periodic surface structures. Adsorption studies on single crystal surfaces provide information on site specific (e.g., a-top, bridge, or hollow) adsorption and occupation, as well as the formation of new chemical phases on surfaces upon exposure to gases. In addition, by changing surface orientation and/or miss-cut angle to form vicinal surfaces with varying step densities, factors such as face specificity (e.g. (100) versus (111)) of adsorption and reactions or the role of defects can be systematically addressed. APXPS experiments on single crystal surfaces allow investigation of these molecular-level properties as pressure is increased above UHV and therefore provide a direct connection between the vast knowledge gained from decades of research under UHV conditions to how these 
systems evolve at elevated pressures. Such detailed information may be more difficult to obtain on structurally more complex systems. Here we provide a few examples of recent APXPS results on welldefined single crystal surfaces.

\section{CO adsorption on Ru(0001): Increased coverage and new adsorption sites at elevated pressures}

Increasing the pressure beyond UHV conditions extends the thermodynamic phase-space of the adsorbate-surface system that can be explored with XPS. Among the simplest consequences of this extension are an increase in adsorbate coverage and the occupation of new surface adsorption sites. Recently, Starr et al. ${ }^{100}$ demonstrated that at $300 \mathrm{~K}$, when the pressure of $\mathrm{CO}$ is increased above approximately $10^{-6} \mathrm{mbar}$, the surface coverage of $\mathrm{CO}$ on $\mathrm{Ru}(0001)$ increases beyond that observed under UHV conditions, where the maximum observed $\mathrm{CO}$ coverage on $\mathrm{Ru}(0001)$ is $0.67 \mathrm{ML}$ and the adsorbed $\mathrm{CO}$ is located exclusively in Ru a-top sites. ${ }^{216,217}$ Starr et al. observed that at elevated pressures, the coverage of $\mathrm{CO}$ saturated at approximately $0.88 \mathrm{ML}$ for $\mathrm{CO}$ pressures above $10^{-2}$ mbar. $\mathrm{O} 1 \mathrm{~s}$ binding energy shifts indicate that most of the additional $\mathrm{CO}$ adsorbed at elevated pressures is located in bridge sites on the $\mathrm{Ru}(0001)$ surface. $\mathrm{CO}$ adsorption on $\mathrm{Ru}(0001)$ in any high symmetry sites other than a-top had not been previously observed under UHV conditions. Using isobaric measurements in 0.05 mbar of $\mathrm{CO}$, Starr et al. found that when increasing the temperature above $350 \mathrm{~K}$ the bridge bound $\mathrm{CO}$ begins to desorb from the surface and is completely desorbed at temperatures above $400 \mathrm{~K}$. Previous infrared spectroscopy experiments carried out by Hoffmann et al. observed only a-top bound CO for CO pressures up to 13 mbar, but in the temperature range of 500 to $700 \mathrm{~K} \cdot{ }^{218,219}$ These combined results demonstrate that Starr et al. were likely probing an unexplored part of thermodynamic phase space and that a pressure gap exists between previous UHV studies and elevated pressure studies for the $\mathrm{CO} / \mathrm{Ru}(0001)$ system.

\section{$\mathrm{H}_{2} \mathrm{O}$ adsorption on $\mathrm{Cu}(110)$ and $\mathrm{Cu}(111)$ surfaces: surface orientation dependence of wetting}

The adsorption of water on the (110) and (111) surfaces of $\mathrm{Cu}$ provides a stark example of how surface orientation may determine its adsorption properties. The adsorption and reaction of water on $\mathrm{Cu}$ 
catalysts is important in a variety of industrial important reactions including the water gas shift reaction, and the synthesis of methanol. ${ }^{220,221}$ As a result the adsorption of water on $\mathrm{Cu}$, particularly $\mathrm{Cu}(110)$, has been studied extensively using UHV surface science techniques..$^{222,223,224,225,226,227,228,229,230,231,232,233,234}$ At UHV conditions and low temperatures, water adsorbs molecularly forming a $(7 \times 8)$ unit cell at one monolayer coverage. Upon heating this monolayer to $170 \mathrm{~K}$ a mixed $\mathrm{OH}$ and $\mathrm{H}_{2} \mathrm{O}$ phase has been observed, indicating partial dissociation of $\mathrm{H}_{2} \mathrm{O}$. Interestingly, the dissociation barrier of water is lower for the monolayer by approximately 0.3 to $0.4 \mathrm{eV}$ compared to that of an isolated water molecule, pointing to the important role that hydrogen bonding plays in the dissociation of water on $\mathrm{Cu}(110)$.

Recent APXPS experiments on the $\mathrm{H}_{2} \mathrm{O} / \mathrm{Cu}(110)$ system have explored the adsorption of water at close to ambient relative humidities. ${ }^{114,115} \mathrm{O} 1 \mathrm{~s}$ spectra collected at 1.3 mbar water vapor pressure and temperatures ranging from $275 \mathrm{~K}$ to $520 \mathrm{~K}$ (corresponding to relative humidity, $\mathrm{RH}$, from $19 \%$ to 0.003 $\%)$ indicated the presence of pure $\mathrm{OH}(\mathrm{RH}<\sim 0.01 \%)$ and mixed $\mathrm{H}_{2} \mathrm{O} / \mathrm{OH}$ phases $(\mathrm{RH}>\sim 0.01 \%)$. The presence of water molecules at such low $\mathrm{RH}$ was attributed to $\mathrm{H}$-bonding between the $\mathrm{OH}$ groups and water molecules. The results of both UHV studies and AP-XPS studies for $\mathrm{H}_{2} \mathrm{O}$ adsorption on $\mathrm{Cu}(110)$ have emphasized the important role that $\mathrm{OH}$ plays in stabilizing molecular water on the $\mathrm{Cu}(110)$ surface through hydrogen bond formation.

The importance of $\mathrm{OH}$ groups in stabilizing molecular water adsorption on $\mathrm{Cu}$ surfaces is directly illustrated by comparing APXPS results for the adsorption of water on the $\mathrm{Cu}(110)$ surface to those on $\mathrm{Cu}(111)$ (see Figure 4). ${ }^{117}$ For a relative humidity up to $32 \%(1.3 \mathrm{mbar}, 268 \mathrm{~K})$ the $\mathrm{Cu}(111)$ surface remains free of both molecular $\mathrm{H}_{2} \mathrm{O}$ and $\mathrm{OH}$. This is a direct consequence of the higher $\mathrm{H}_{2} \mathrm{O}$ dissociation barrier of $\sim 0.3 \mathrm{eV}$ on the (111) surface compared to the (110) surface. The kinetically hindered dissociation of $\mathrm{H}_{2} \mathrm{O}$ on $\mathrm{Cu}(111)$ does not allow the formation of adsorbed $\mathrm{OH}$ which act as anchoring sites for molecular $\mathrm{H}_{2} \mathrm{O}$ adsorption. By pre-adsorbing atomic oxygen on $\mathrm{Cu}(111), \mathrm{OH}$ groups can be formed on the surface upon exposure to water, which leads to the observation of both $\mathrm{OH}$ and molecular $\mathrm{H}_{2} \mathrm{O}$ at 1.3 mbar and $295 \mathrm{~K}$ (see Figure 4). The difference in water adsorption properties on the $\mathrm{Cu}(111)$ 
and $\mathrm{Cu}(110)$ surfaces is a direct consequence of the different activation energies for water dissociation on these surfaces.

These studies highlight the influence of the surface crystallography on the surface chemistry. Depending on the specific catalytic reaction mechanism, these results may have profound implications for heterogeneous catalysis on $\mathrm{Cu}$ and other metal surfaces. For reactions that require the formation of $\mathrm{OH}$ groups, the reaction may be effectively poisoned if water molecules bind to $\mathrm{OH}$ and block access for other molecules to the adsorbed $\mathrm{OH}$. Similarly, the lack of ability for $\mathrm{Cu}(111)$ to dissociate $\mathrm{H}_{2} \mathrm{O}$ to form $\mathrm{OH}$ without the presence of adsorbed oxygen may lead to decreased reactivity for those catalysts containing predominantly (111) facets.

\section{APXPS experiments of CO adsorption and oxidation over Pt single crystal and vicinal surfaces}

The use of platinum as a catalyst dates back well over a century. Because of this many early surface science studies focused on the adsorption and reaction of simple molecules on single crystal surfaces of $\mathrm{Pt}$, in particular $\mathrm{CO}$ oxidation. The Pt surface is also known to be quite dynamic. For example the clean $\operatorname{Pt}(100)$ surface reconstructs into a $(1 \times 5)$ structure under UHV conditions. ${ }^{235}$ Surface reconstructions lower the surface free energy of the surface; adsorbates often lift such reconstructions due to a reduction in the free energy of the adsorbate-surface system. ${ }^{236}$ When a surface is in equilibrium with the gas phase, the chemical potential of the gas phase must be considered. While at low pressure conditions this is a small quantity and contributes little to the system's energetics, at elevated pressures its contribution may be significant. For example, when increasing the pressure from $10^{-10}$ mbar to 1 mbar the gas phase chemical potential increases by $10 \mathrm{RT}$, or $25 \mathrm{~kJ} / \mathrm{mol}$, at $300 \mathrm{~K}$, a non-negligible change in the free energy of the surface layer. The nature of Pt surface restructuring depends intimately on the type of catalytic reaction, temperature, pressure, and gas composition. Due to the possibility of high adsorbate coverages at pressures above UHV, elevated pressure conditions may lead to a decrease in the activation barriers for surface restructuring and thus to reaction pathways not observed at UHV. Recent elevated pressure surface sensitive techniques have begun to address the complex behavior of the Pt surface at 
reaction conditions, specifically addressing surface reconstructions, the chemical nature of adsorbates on the surface, as well as their effects on reaction mechanisms. Here we highlight two recent APXPS experiments on the adsorption and reaction of $\mathrm{CO}$ on Pt surfaces.

Tao et al. recently investigated the restructuring of stepped Pt surfaces, (specifically (557) and (332)) at CO pressures up to 0.7 mbar. ${ }^{67}$ Using AP-XPS they determined that at 0.7 mbar the CO coverage is approximately one monolayer. This is nearly twice the amount of $\mathrm{CO}$ adsorbed on the $\mathrm{Pt}(557)$ surface at $7 \times 10^{-9} \mathrm{mbar}$. Along with the increase in CO coverage, $\mathrm{O} 1 \mathrm{~s}$ and $\mathrm{Pt} 4 \mathrm{f}$ spectra showed a substantial increase in intensity at $533.1 \mathrm{eV}(\mathrm{O} 1 \mathrm{~s})$ and $72.15 \mathrm{eV}(\mathrm{Pt} 4 \mathrm{f})$ binding energies (see Figure 5). These binding energies are higher than those observed for $\mathrm{CO}$ adsorbed in Pt a-top sites. ${ }^{237}$ In general, O 1s and $\mathrm{C} 1 \mathrm{~s}$ binding energies shift to higher values as the coordination of $\mathrm{CO}$ to the surface decreases. For example, on $\mathrm{Pt}(111)$ the $\mathrm{O} 1 \mathrm{~s}$ binding energy of $\mathrm{CO}$ bound to Pt bridge sites is $531.0 \mathrm{eV}$ as compared to $532.7 \mathrm{eV}$ for a-top bound $\mathrm{CO}{ }^{237}$ Similarly, a lower coordinated Pt atom should lead to higher Pt $4 \mathrm{f}$ binding energies. Thus, the higher binding energies observed by Tao et al. are consistent with CO bound to low-coordinated Pt sites. The additional features observed in the Pt $4 \mathrm{f}$ and $\mathrm{O}$ 1s spectra were reversible as evidenced by the consistent changes in peak intensity as the pressure was cycled between $7 \times 10^{-9} \mathrm{mbar}$ and 0.7 mbar. The observed $\mathrm{CO}$ coverage changed from $\sim 0.5$ to 1.0 , respectively, at those pressures. Complimentary STM experiments indicated a dramatic surface restructuring at pressures above $0.1 \mathrm{mbar}$ and the formation of triangular nanoclusters of approximately $2.2 \mathrm{~nm}$ by $2.1 \mathrm{~nm}$ in size (see Figure 5 ) ${ }^{67}$ The formation of these nanoclusters leads to an increase in the number of under-coordinated Pt atoms on the surfaces, which act as new adsorption sites for $\mathrm{CO}$ at elevated pressures. Such dramatic restructuring of the $\mathrm{Pt}$ (557) surface was proposed to be driven by a relaxation of repulsive $\mathrm{CO}-\mathrm{CO}$ interactions as the CO coverage increased to nearly 1.0 and confirmed by DFT calculations. These results highlight the dynamic nature of Pt at elevated pressure conditions. Further, the level of detail in these studies provides a molecular-level understanding of the mechanism responsible (i.e., $\mathrm{CO}-\mathrm{CO}$ repulsion) for dynamic changes at the surface under reaction conditions. Extending these kind of investigations to structurally 
more complex model and technical catalysts may provide new insights into the dynamic nature of the Pt surface and its role in the reactivity of supported Pt catalysts.

Another APXPS study has addressed CO oxidation over Pt(110) at elevated pressures, motivated by earlier high-pressure STM and gas analysis investigations by Hendriksen et al. who observed a roughening of the $\mathrm{Pt}(110)$ surface during $\mathrm{CO}$ oxidation, which was correlated to an enhanced rate of $\mathrm{CO}_{2}$ production. ${ }^{238}$ This roughening occurred at high $\mathrm{O}_{2} / \mathrm{CO}$ ratios of $>45$, pressures of $\sim 0.5$ bar, and temperatures of $425 \mathrm{~K}$ and was therefore assumed to be associated with the formation of Pt-oxide. Hendriksen et al. concluded that, at high pressures and $\mathrm{O}_{2} / \mathrm{CO}$ ratios, $\mathrm{CO}$ oxidation may follow the MarsVan Krevelen mechanism as opposed to the Langmuir-Hinshelwood mechanism. Recent APXPS experiments on this system, however, indicate that the actual mechanism responsible for CO-oxidation over Pt(110) may be sensitive to the precise conditions. ${ }^{70}$ Chung et al. used APXPS to study CO oxidation over $\mathrm{Pt}(110)$ at a variety of $\mathrm{CO}$ and $\mathrm{O}_{2}$ pressures and temperatures. ${ }^{70}$ When 0.26 mbar of $\mathrm{CO}$ is introduced into the chamber at room temperature both $\mathrm{C} 1 \mathrm{~s}$ and $\mathrm{O} 1 \mathrm{~s}$ spectra show the presence of $\mathrm{CO}$ adsorbed in both a-top and bridge sites in agreement with previous UHV studies. Upon addition of 0.26 mbar of $\mathrm{O}_{2}$ at room temperature the population of bridge sites decreased, and continued to decrease even further when the $\operatorname{Pt}(110)$ crystal was heated to $100{ }^{\circ} \mathrm{C}$, when nearly all bridge-bound $\mathrm{CO}$ was removed. $\mathrm{CO}_{2}$ production began at about $120{ }^{\circ} \mathrm{C}$. At $150{ }^{\circ} \mathrm{C} \mathrm{CO}_{2}$ is still produced and the surface remained $\mathrm{CO}$ covered and there was no observation of either chemisorbed oxygen or Pt-oxide.

Chung et al. addressed the possibility of the presence of chemisorbed oxygen or Pt-oxide by exploring the effects of different $\mathrm{O}_{2} / \mathrm{CO}$ ratios at $150{ }^{\circ} \mathrm{C}$ on the $\mathrm{CO}$ coverage. Introduction of 0.23 mbar of $\mathrm{O}_{2}$ at $150{ }^{\circ} \mathrm{C}$ in the absence of $\mathrm{CO}$ created a $\mathrm{Pt}(110)$ surface covered with chemisorbed oxygen. Upon introduction of $\mathrm{CO}$ to 0.30 mbar, $\mathrm{CO}_{2}$ production instantly increased but then decreased with time. Once $\mathrm{CO}_{2}$ production stabilized, $\mathrm{O} 1 \mathrm{~s}$ spectra revealed that the chemisorbed oxygen was completely removed. This demonstrates that an oxygen covered $\mathrm{Pt}(110)$ surface is not stable under $\mathrm{CO}$ rich conditions, but that the oxygen-covered surface is more reactive than a $\mathrm{CO}$ covered surface due to the higher rate of $\mathrm{CO}_{2}$ production at short time intervals following the introduction of CO. Upon reduction of the CO pressure 
to 0.18 mbar (i.e., in a more $\mathrm{O}_{2}$ rich environment), the rate of $\mathrm{CO}_{2}$ production increased but the $\mathrm{Pt}$ surface remained covered with $\mathrm{CO}$, although at a slightly lower coverage. At these conditions no oxygen or Ptoxide was observed in the $\mathrm{O}$ 1s spectra. These results demonstrate that even under $\mathrm{O}_{2}$ rich conditions at these pressures $\mathrm{CO}$ oxidation may still occur via a Langmuir-Hinshelwood mechanism. However, these results should not be taken as definitive evidence that CO-oxidation occurs via a Langmuir-Hinshelwood mechanism, but instead that the precise reaction mechanism is sensitive to the $\mathrm{O}_{2} / \mathrm{CO}$ ratio as well as total pressure.

The electronic structure of oxygen species on Ag catalysts

The interaction of oxygen with silver has been studied extensively over the past years, mainly because of the importance of silver-based catalysts in the epoxidation of ethylene ${ }^{239}$ and partial oxidation of methanol to formaldehyde ${ }^{240}$. These two reactions are part of large scale production processes in the chemical industry. However, the reaction mechanism in either of these cases is still under debate, partially due to the limited availability of results from in situ measurements.

Recent APXPS measurements have addressed these issues. The $\mathrm{O}$ 1s core level spectra of both a $\mathrm{Ag}(110)$ single crystal surface and of a $\mathrm{Ag}$ powder sample (nominal particle size $45 \mu \mathrm{m}$ ) are shown in Figure $6 .^{91}$ The oxygen species at the surface of both catalysts change with the sample temperature and, in the case of the powder sample, also with the exposure time to $0.25 \mathrm{mbar}_{2}$ at $180^{\circ} \mathrm{C}$. At the lowest temperature $\left(150^{\circ} \mathrm{C}\right)$ the most abundant species at both surfaces is $\mathrm{O}_{\alpha 1}$, which is associated with the formation of a $\mathrm{p}(4 \times 4)$ oxygen overlayer. At higher temperature three other oxygen species become more prominent $^{241} . \mathrm{O}_{\alpha 2}$ (nucleophilic oxygen) is an oxide-like species located at steps on the surface. ${ }^{242}$ It is important for the activation of $\mathrm{C}-\mathrm{H}$ bonds in hydrocarbons and is therefore involved in the total oxidation reaction. The peaks at the higher binding energy, $\mathrm{O}_{\alpha 3}$ (electrophilic oxygen) and $\mathrm{O}_{\beta}$, are assigned to atomically adsorbed oxygen at the $\mathrm{Ag}$ surface ${ }^{88}$ and oxygen located in the subsurface region in the $\mathrm{Ag}$ catalysts $^{243}$, respectively. Electrophilic oxygen activated the $\mathrm{C}=\mathrm{C}$ bond in olefins and is thus involved in selective oxidation reactions. 
To characterize the nature of the different oxygen species, Ag3d core level spectra were measured as well (Figure 7). The preparation of the surfaces was done under conditions favoring mainly the formation of a single oxygen species in the $\mathrm{O} 1 \mathrm{~s}$ spectrum. The amount of ionic silver $\left(\mathrm{Ag}^{+}\right)$as a function of the different oxygen species is shown in the bottom graph in Figure 7. Different oxygen species clearly lead to different levels of charge transfer from the $\mathrm{Ag}$ to the oxygen. The degree of $\mathrm{Ag}^{+}$formation in the presence of $\mathrm{O}_{\alpha 1}$ and $\mathrm{O}_{\alpha 2}$ is much higher than that for electrophilic oxygen, indicating different roles for the different oxygen species in the ethylene epoxidation reaction. The strongly charged oxygen species $\mathrm{O}_{\alpha 1}$ and $\mathrm{O}_{\alpha 2}$ activate the $\mathrm{C}-\mathrm{H}$ bonds, leading to $\mathrm{CO}_{2}$ formation, while the less charged electrophilic oxygen activates the $\mathrm{C}=\mathrm{C}$ bond. The different electronic structure of nucleophilic oxygen $\left(\mathrm{O}_{02}\right)$ and electrophilic oxygen $\left(\mathrm{O}_{\alpha 3}\right)$ is strongly influenced by the subsurface oxygen species $\mathrm{O}_{\beta}$. In the presence of $\mathrm{O}_{\beta}$ there are fewer electrons available that can be transferred to an adsorbed oxygen species: thus, the adsorbed oxygen is less charged and $\mathrm{O}_{\alpha 2}$ is formed. In the absence of subsurface oxygen, more electrons can be transferred from Ag to adsorbed oxygen atoms, leading to the formation of highly charged nucleophilic oxygen. Figure 7 also shows the amount of $\mathrm{Ag}^{+}$as a function of another oxygen species, $\mathrm{O} \gamma$, which is formed at about $500{ }^{\circ} \mathrm{C}$ and is assigned to oxygen atoms replacing $\mathrm{Ag}$ atoms in the surface. Due to the high formation temperature, $\mathrm{O} \gamma$ is only relevant for methanol oxidation. The data in Fig. 6 and 7 demonstrate that under constant oxygen partial pressure and catalytically relevant temperatures the silver-oxygen system shows a dynamic behavior, with the formation of different oxygen species as a result of oxygen incorporation in the subsurface region.

To summarize the examples of APXPS measurements on single crystal samples, these studies highlight the utility of APXPS to provide detailed information on adsorbate-induced restructuring of surfaces, orientation-dependent adsorption properties of surfaces, and the pressure-dependence of surface reaction mechanisms. Studies on single crystal surfaces have the advantage of retaining the molecular or atomic level information available in traditional UHV surface science experiments. The level of detail provided by these studies may have been difficult to achieve on more structurally complex surfaces such 
as model or technical catalysts. Studies on single crystal surfaces at elevated pressures provide a direct means to bridge the pressure gap between UHV surface science experiments and more realistic catalytic operating conditions. We now proceed to discuss measurements on more complex systems.

\section{B) Investigation of nanoparticles used in CVD processes for carbon nanotube CNT growth}

Carbon nanotubes (CNTs) have many potential applications, including supercapacitors ${ }^{244}$, fieldemission devices $^{245}$ and vertical interconnects (vias) for integrated circuits ${ }^{246}$, which require growth of vertically aligned CNTs on electrically conductive substrates. CNTs can be grown using chemical vapor deposition (CVD). ${ }^{247}$ CVD is an established technique to synthesize CNT "forests" (i.e., a dense layer of vertically aligned CNTs) on insulation oxide supports such as silica and alumina. ${ }^{248}$. The growth on conductive substrates such as metals, metal-nitrides, and metal-silicides is more difficult and less studied, mainly because of the much higher surface energy of metals compared to insulating oxides, which inhibits the catalyst film from transforming itself into nanoparticles during temperature treatment ${ }^{249}$. In addition, the metallic support has to retain its conductivity and functionality during the CVD process at elevated temperatures in the presence of reactive gases. However, metals are often reactive under such conditions. ${ }^{125}$ Therefore the support has to fulfill the requirements of favorable surface energetics for high density nanoparticle formation and chemical stability against carbide-formation (from the gas that serves as the carbon source) or oxidation (from residual oxygen or water).

Cobalt-silicides are promising catalysts for the synthesis of CNT forests and were recently investigated using APXPS and in situ X-ray diffraction (XRD) to study the evolution of the silicidecatalyst-gas system during CNT forest growth. The Co silicide was prepared as follows (Figure 8): a 200 $\mathrm{nm}$ thick polycrystalline $\mathrm{Si}$ ("poly-Si") film was deposited by CVD onto a crystalline $\mathrm{Si}(100)$ wafer (not shown). Then a $15 \mathrm{~nm}$ thin layer of Co was sputtered on top of the poly-Si. This structure was capped by a TiN layer and annealed at temperatures below $500^{\circ} \mathrm{C}$ for less than three minutes, followed by the removal of the TiN capping layer. The annealing induces an inter-diffusion of $\mathrm{Co}$ and $\mathrm{Si}$, resulting in the formation of $\mathrm{CoSi}$. The TiN layer permits the development of a rather smooth silicide surface. Usually a 
second annealing step is required to transform CoSi into the highly conductive $\mathrm{CoSi}_{2}{ }^{250}$. The second annealing step can be avoided here, since the transformation of $\mathrm{CoSi}$ to $\mathrm{CoSi}_{2}$ can be done simultaneously with the CNT growth over a pressure range from 0.1 mbar to 1 bar as described below. ${ }^{124}$

After the deposition of a $1 \mathrm{~nm}$ thick Fe layer the evolution of the sample was studied by APXPS during the CVD process in the mbar range. Figure 9 shows XPS spectra (Si 2p, Co 2p and Fe 2p) during the CVD process. The as-loaded surface consists of Co oxide and Si oxide, since the samples were exposed to air during the transport between process steps. The Fe film is completely oxidized as well. The Si $2 \mathrm{p}$ spectra show an intensity increase when the sample is heated to $650{ }^{\circ} \mathrm{C}$ in $\mathrm{NH}_{3}$. This treatment is required in order to reduce the Fe nanoparticles, since only Fe metal catalyzes the growth of CNTs. The intensity increase is due to the reduction of the Co oxide and the removal of $\mathrm{C}$ contamination on the surface. A new peak at a $\mathrm{BE}$ of $99.7 \mathrm{eV}$ indicates the formation of $\mathrm{CoSi}_{2}$. However, most of the $\mathrm{Si}$ remains oxidized. The Co is nearly completely reduced. A shoulder at the high binding energy side of the metallic Co peak at $778.9 \mathrm{eV}$ indicates the formation of $\mathrm{CoSi}_{2}$ which forms nano-crystalline domains. ${ }^{130}$

During annealing the Fe film decomposes into nanoparticles. In contrast to the case where $\mathrm{Fe}$ is supported on and $\mathrm{Al}_{2} \mathrm{O}_{3}$ substrate, here only part of the Fe particles are reduced to metal by the treatment in $\mathrm{NH}_{3}$ (note the peak at $706.9 \mathrm{eV}$ in the $\mathrm{Fe} 2 \mathrm{p}$ spectrum in Figure 9). The Co silicide support keeps a portion of the Fe nanoparticles in their oxidized state. The combination of Co silicide substrate and $\mathrm{Fe}$ catalysts is one of the most promising methods for high density CNT growth on insulators to date. The interfacial oxide layer prevents the catalyst nanoparticles to diffuse onto the surface and to agglomerate to bigger clusters. An atomic force microscopy study has shown that $\mathrm{CoSi}_{2}$ inhibits sintering of the $\mathrm{Fe}$ nanoparticles, which facilitates efficient growth of CNT forests. ${ }^{251}$ The APXPS results shown in Figure 9 indicate that the $\mathrm{Fe}$ nanoparticles are bound to the $\mathrm{CoSi}_{2}$ substrate through a similar interfacial interaction as observed for $\mathrm{Fe}$ nanoparticles supported on $\mathrm{Al}_{2} \mathrm{O}_{3}$.

The addition of $10 \% \mathrm{C}_{2} \mathrm{H}_{2}$ to the $\mathrm{NH}_{3}$ (top spectra in Figure 9) results in the fast evolution of $\mathrm{sp}^{2}$ and $\mathrm{sp}^{3}$ type bonds in the $\mathrm{C} 1 \mathrm{~s}$ spectra, indicating $\mathrm{CNT}$ growth. The growth rate under the experimental conditions was too fast to measure high resolution APXP spectra during $\mathrm{C}_{2} \mathrm{H}_{2}$ exposure. Constant 
exposure of the sample to $\mathrm{C}_{2} \mathrm{H}_{2}$ leads to CNT forests with thicknesses of up to $40 \mu \mathrm{m}$, much thicker than the information depth in APXPS experiments. To monitor the chemical state of the interface during growth, short pulses of $\mathrm{C}_{2} \mathrm{H}_{2}\left(10^{-2} \mathrm{mbar}\right.$ for $\left.10 \mathrm{~s}\right)$ were admitted to the chamber, which allows only sparse growth of CNTs, but enables probing the silicide surface. After a $\mathrm{C}_{2} \mathrm{H}_{2}$ pulse this surface is comprised of metallic $\mathrm{Co}, \mathrm{CoSi}_{2}$, and some $\mathrm{SiO}_{2}$, with the state of $\mathrm{Fe}$ not affected by $\mathrm{C}_{2} \mathrm{H}_{2}$ exposure. XRD measurements (not shown) of the same sample reveal the exclusive presence of $\mathrm{Si}$ and $\mathrm{CoSi}_{2}$, thus proving that the oxides observed in the APXP spectra are located only at the surface and do not extend into the bulk of the film.

\section{C) Application of APXPS to electrochemistry}

The need for clean, secure, and sustainable energy sources has created a surge in research and development of electrochemical devices, such as batteries, fuel cells, and super capacitors. Many roadblocks to higher performing electrochemical devices are not just due to engineering challenges, but also due to limited information on the fundamental processes in electrochemical devices at the molecular level, which requires experimental tools for observing electro-chemical processes directly at the interfaces where they occur in situ. Fuel cells, which were invented more than 100 years ago, are a case in point. Solid oxide fuel cells (SOFC) in particular offer several key advantages, including high efficiency, high tolerance to poisoning of the catalysts, reformation of hydrocarbon fuels, and the possibility of burning hydrocarbon fuels directly; however, despite these attractive features SOFCs have not yet found widespread use in everyday applications and devices.

Traditional electrochemical evaluation of electrode overpotentials employs, e.g., voltammetry and electrochemical impedance spectroscopy. These techniques provide valuable information on the global electrode overpotentials and resistances in SOFCs. Despite these advances in electrochemical measurement and modeling, our understandings of the rate limiting steps in SOFCs, in particular the cathode oxygen reduction mechanism, the physics governing electrode overpotential losses, and dimensions of the electrochemically active regions of mixed ionic electronic conducting electrodes 
remain largely circumstantial to date. Many of these challenges are due to the inherently convoluted nature of electrochemical and chemical processes and the lack of suitable in situ techniques to probe these issues at relevant temperatures and pressures. As pointed out by Adler in 2004: "New in situ analytical techniques are needed, particularly which can be applied at ambient pressures, that can probe what is happening in an electrode as a function of temperature, $\mathrm{P}_{\mathrm{O} 2}$, polarization, local position, and time." ${ }^{, 252}$

To address these challenges using photoelectron spectroscopy, scientists from the ALS, University of Maryland, and Sandia National Laboratory began using APXPS as an operando tool to study solid oxide electrochemical cells (SOCs) in 2008. APXPS allows the study of the surfaces in situ with elemental and chemical specificity. By scanning a focused X-ray spot across the surface or by using an imaging mode of the photoelectron spectrometer, local elemental and chemical information across the sample surface can be obtained. In addition, local electrical potential changes at the surface can be determined from the changes of the kinetic energy of core level photoelectron peaks. The correlation of local chemical processes with local electrical potentials under operating conditions is crucial for an understanding of fundamental processes in SOC devices.

The first experiments were performed on a SOC cell in which a Au-ceria working electrode (WE) and a Pt counter electrode (CE) were deposited on a single crystal YSZ electrolyte disk. In such a planar cell design, all components are exposed to the surrounding gas atmosphere and located on the same side of the electrolyte disk to enable APXPS access the electrode-electrolyte interfaces. Since the oxidizer and fuel are in the same volume, a bias is applied between the Pt CE and ceria WE to drive the electrochemical reactions. This cell was mounted inside the APXPS endstation at beamline 11.0.2 at the $\mathrm{ALS}^{24}$ and heated up to $750^{\circ} \mathrm{C}$ in a $1: 1$ gas mixture of $\mathrm{H}_{2}$ and $\mathrm{H}_{2} \mathrm{O}$ at a total pressure of $\sim 1.3$ mbar. The results of these measurements are shown in Figure 10 and prove the validity of the experimental concept: a clear correlation between gradients in the electrical potential and changes in the surface chemistry (namely the Ce oxidation state) is observed. ${ }^{180}$

These techniques were subsequently applied to an new version of a model Ceria-YSZ-Pt SOC ${ }^{178}$ and Ni-YSZ-Pt SOC ${ }^{182}$. A new endstation at ALS Beamline 9.3.2 ${ }^{25}$ and a special sample holder were 
utilized as well. ${ }^{43}$ In all of these experiments, the WE (ceria/Au or Ni) was grounded and the bias voltage was applied to the Pt CE using a potentiostat. Two-probe linear sweep voltammetry and electrochemical impedance spectroscopy experiments were conducted simultaneously to the APXPS measurements.

Figure 10a shows the schematic layout of a planar ceria/YSZ/Pt cell geometry and simplified experimental setup. ${ }^{178}$ Ceria working electrodes of different thicknesses $(50,100$, and $250 \mathrm{~nm})$ are sputtered onto a gold current collector and only extend onto the YSZ electrolyte towards the Pt counter electrode. Such a cell design mandates that oxygen ions move in the vertical direction through ceria and electrons (polarons) move in the lateral direction across the ceria. Therefore, the ionic and electronic potential changes can be separated and measured individually. Using this specially fabricated single chamber SOC, the authors of Ref. 178 have demonstrated that the active electrochemical region on ceria extends $150 \mu \mathrm{m}$ away from the current collector and that significant shifts from the equilibrium surface $\mathrm{Ce}^{3+} / \mathrm{Ce}^{4+}$ concentrations are needed to drive the electro-oxidation of $\mathrm{H}_{2}$ and the electrolysis of $\mathrm{H}_{2} \mathrm{O}$ (see Figure 10c). The correlation between local potential losses and local chemical state changes were obtained directly from working SOC devices.

Figure 11 is taken from a study of a Ni-YSZ-Pt SOC, ${ }^{182}$ where the new endstation at ALS Beamline 9.3.2 was used. ${ }^{25}$ The spectrometer was optimized in this project to perform 1D spatiallyresolved APXPS. These measurements probed the individual overpotentials (such as between Ni and YSZ, YSZ and Pt) in SOC devices, allowing a direct correlation of changes in the individual overpotentials with the applied bias in terms of the different electro-catalytic activities of $\mathrm{Ni}$ and $\mathrm{Pt}$ for the $\mathrm{H}_{2} \mathrm{O}$ splitting and $\mathrm{H}_{2}$ oxidation reactions. It was found that $\mathrm{H}_{2} \mathrm{O}$ splitting is faster than $\mathrm{H}_{2}$ oxidation on $\mathrm{Ni}$, while on $\mathrm{Pt}$ the $\mathrm{H}_{2}$ oxidation reaction proceeds more rapidly than $\mathrm{H}_{2} \mathrm{O}$ splitting.

APXPS is a unique non-contact tool to probe electrode/gas and electrode/electrolyte interfaces as a function of temperature, pressure, polarization, local position, and time, which makes it also an excellent method to study fundamental processes in model battery devices, such as $\mathrm{Li}^{-} \mathrm{O}_{2}$ battery cells. ${ }^{151}$ The combination of local measurements of the surface chemistry and electrical potentials using APXPS with bulk measurements of the device performance using voltammetry and electrochemical impedance 
spectroscopy is a promising strategy for gathering fundamental mechanistic information on electrochemical devices which may facilitate advances in the design of electrochemical devices.

\section{Conclusions and outlook}

Ambient pressure X-ray photoelectron provides a wealth of information on vapor/solid interfaces under reaction conditions, from the elemental composition and chemical specificity (oxidation state, functionalization), to the local electrical potentials and work functions. As the preceding examples and Table 1 demonstrate, this allows molecular scale investigations of interfacial phenomena in a wide range of scientific areas, including fundamental surface science, environmental science, electrochemistry, and industrial catalysis. As the technique has matured and broadened its user base over the last decade (both in total numbers of investigators as well as in the breadth of applications), mainly through the commissioning of new endstations at synchrotrons and now increasingly through the installation of new laboratory-based instruments, the task at hand is the further development of in situ cells that allow to measure samples under more realistic and complex experimental conditions, and combining APXPS with simultaneous measurements using other techniques to, e.g., monitor surface as well as bulk properties, and correlate the surface chemistry with the gas phase composition.

Several new developments promise to expand APXPS to study phenomena that have hitherto been difficult or impossible to investigate:

1) High kinetic energy APXPS (with photoelectron kinetic energies exceeding $5 \mathrm{keV}$ ) utilizes the increased mean free path of electrons with increasing KE. This will allow to study the chemistry of the subsurface region under reaction conditions, which may differ from that of the surface and plays an important role in heterogeneous catalysis and liquid/vapor reactions. Vacuum-based high kinetic energy XPS has already been used to investigate buried interfaces at depths larger than $10 \mathrm{~nm} .^{253}$ One of the most important buried interfaces is that between a solid and a liquid, which drives many processes in electrochemistry, corrosion, and environmental science. ${ }^{254}$ The inelastic mean free path of electrons in liquid water is about $20 \mathrm{~nm}$ at $10 \mathrm{keV}$ kinetic energy, making it feasible to penetrate about 70 monolayers 
of water, thus approaching conditions at bulk water/solid interfaces. ${ }^{255}$ The preparation of thin water films with thicknesses of 10 to $20 \mathrm{~nm}$ is an experimental challenge, though. Another advantage of high kinetic energy APXPS is the reduced scattering of photoelectrons in the gas phase, which will indeed make it possible to obtain XPS spectra at atmospheric pressure.

2) Increased spatial resolution in APXPS is crucial for the understanding of the complex chemistry at the surface of multi-component samples and devices, such as supported catalysts, electrochemical devices, as well as natural mineral and aerosols. The spatial resolution in APXPS experiments is in general determined by the dimension of the incident X-ray beam, which usually is on the order of several 10 to several $100 \mu \mathrm{m}$, or by the spatial resolution of an area detector (where spatial resolution is only available in one dimension). In general APXPS spectra average over the entire area that is illuminated by the incident X-rays or that is within the field of view of the electron spectrometer, thus convoluting contributions from different components of the heterogeneous surface, which complicates the determination of the roles of the various parts of the sample surface to the overall reactivity. A straightforward method for the improvement of the spatial resolution is to tightly focus the X-ray beam using either refocusing mirrors (such as Kirkpatrick-Baez type mirror pairs that have demonstrated a spatial resolution below $50 \mathrm{~nm}$ for hard X-rays ${ }^{256}$ ) or Fresnel zone plates with a spatial resolution of currently less than $10 \mathrm{~nm} .{ }^{257}$ More tightly focused incident X-ray beams will increase the flux density at the sample surface, with a concomitant chance of beam-induced damage to the sample surface, which needs to be mitigated in those experiments.

3) In addition to increased spatial resolution, the investigation of heterogeneous chemical processes at surfaces over a wide range of time scales will be of increasing importance in many fields of research. Areas of interest include the kinetics of low-temperature oxidation of metals and oxides (minutes to hours) on the slow side to the observation of intermediate species in heterogeneous catalytic reactions, which requires a temporal resolution on the nanosecond scale or better. The latter is particularly challenging since the time-averaged concentration of reaction intermediates is low under catalytically-relevant conditions, where it is difficult to observe these states using XPS, which has a 
sensitivity of usually not better than a few percent of a monolayer. Pump-probe experiments using, e.g., $\mathrm{THz}$ excitation, combined with fast probes (i.e., delay-line detectors) may provide a path to study these phenomena on the relevant time scales of catalytic reactions, opening up the possibility to detect the fundamental steps in a heterogeneous chemical reactions at relevant pressures and temperatures.

\section{Acknowledgements}

The Advanced Light Source and beamline 11.0.2 are supported by the Director, Office of Energy Research, Office of Basic Energy Sciences, and Chemical Sciences Division of the U.S. Department of Energy under contracts No. DE-AC02-05CH11231. This work was also supported by the Office of Science, Biological and Environmental Research, Environmental Remediation Sciences Division (ERSD), U.S. Department of Energy under Contract No. DE-AC02-05CH11231. DES acknowledges support through the Silicon In-situ Spectroscopy@the Sychrotron (SISSY) project within the Bundesministerium für Bildung und Forschung (BMBF project number 03SF0403). 


\section{References}

1 G. Ertl, Nobel Lecture, http://www.nobelprize.org/nobel_prizes/chemistry/laureates/2007/ertllecture.html.

2 C.S. Subhash and K. Keven, (Eds.), High Temperature and Solid Oxide Fuel Cells, Elsevier, Oxford, U.K., 2003.

3 B.J. Finlayson-Pitts and James N. Pitts, Jr., Chemistry of the Upper and Lower Atmosphere, Academic Press, London, 2000.

$4 \quad$ F. Dominé and P.B. Shepson, Science, 2002, 297, 1506.

$5 \quad$ S.J. Peters and G.E. Ewing, J. Phys. Chem. B, 1997, 101, 10880.

6 V. Sadtchenko, G.E. Ewing, D.R. Nutt and A.J. Stone, Langmuir, 2002, 18, 4632.

$7 \quad$ Y.R. Shen, Nature, 1989, 337, 519.

$8 \quad$ G. Rupprechter, MRS Bulletin, 2007, 34, 1031.

9 J. Forsberg, L.C. Duda, A. Olsson, T. Schmitt, J. Andersson, J. Nordgren, J. Hedberg, C. Leygraf, C. Asstrup, D. Wallinder and J.-H. Guo, Rev. Sci. Instrum., 2007, 78, 083110.

10 S. Ferrer, M.D. Ackermann and E. Lundgren, MRS Bulletin, 2007, 32, 1010.

11 G. Binnig, C.F. Quate and Ch. Gerber, Phys. Rev. Lett., 1986, 56, 930.

12 J. Hu, X.-d. Xiao, D.F. Ogletree and M. Salmeron, Science, 1995, 268, 267.

13 B.L.M. Hendriksen and J.W.M. Frenken, Phys. Rev. Lett., 2002, 89, 046101.

14 T.W. Hansen, J.B. Wagner, P.L. Hansen, S. Dahl, H. Topsøe and C.J.H. Jacobsen, Science, 2001, 294, 1508 .

15 A.M. Donald, Nat. Mat., 2003, 2, 511.

16 S. Hüfner, Photoelectron Spectroscopy, Springer, Berlin, 1995.

17 M. P. Seah and W. A. Dench, Surf. Interface Anal., 1979, 1, 2.

18 H. Siegbahn and K. Siegbahn, J. Electron Spectrosc. Relat. Phenom., 1973, 2, 319.

19 H. Siegbahn, J. Phys. Chem., 1985, 89, 897.

20 R.W. Joyner and M.W. Roberts, Surf. Sci., 1979, 87, 501.

21 H.-J. Ruppender, M. Grunze, C.W.Kong and M. Wilmers, Surf. Interface Anal., 1990, 15, 245.

22 D.F. Ogletree, H. Bluhm, G. Lebedev, C.S. Fadley, Z. Hussain and M. Salmeron, Rev. Sci. Instrum., 2002, 73, 3872.

23 H. Bluhm, M. Hävecker, A. Knop-Gericke, D. Teschner, E. Kleimenov, V.I. Bukhtiyarov, D.F. Ogletree, M. Salmeron and R. Schlögl, J. Phys. Chem. B., 2004, 108, 14340.

24 D.F. Ogletree, H. Bluhm, E.L.D. Hebenstreit and M. Salmeron, Nucl. Instrum. Meth. A, 2009, 601, 151 .

25 M.E. Grass, P.G. Karlsson, F. Aksoy, M. Lundqvist, B. Wannberg, B.S. Mun, Z. Hussain and Z. Liu, Rev. Sci. Instrum., 2010, 81, 053106.

26 F. Aksoy, M.E. Grass, S.H. Joo, N. Jabeen, Y.P. Hong, Z. Hussain, B.S. Mun, Z. Liu, Nucl. Instrum. Meth. A, 2011, 645, 260.

27 S. Kaya, H. Ogasawara, L.-Å. Näslund, J.-O. Forsell, H. Sanchez Casalonguea, D.J. Miller and A. Nilsson, Cat. Today, in press, 2013. 
28 J. Schnadt, J. Knudsen, J.N. Andresen, H. Siegbahn, A. Pietzsch, F. Hennies, N. Johansson, N. Mårtensson, G. Öhrwall, S. Bahr, S. Mähl and O. Schaff, J. Synchr. Rad., 2012, 19, 701.

29 R. Toyoshima, M. Yoshida, Y. Monya, Y. Kousa, K. Suzuki, H. Abe, B.S. Mun, K. Mase, K. Amemeiya and H. Kondoh, J. Phys. Chem. C, 2012, 116, 18691; Supporting Information: http://pubs.acs.org/doi/supp1/10.1021/jp301636u/suppl_file/jp301636u_si_001.pdf

Specs Surface Nano Analysis GmbH, Berlin, Germany. http://www.specs.de/cms/front_content.php?idcat=269

31 VG Scienta, Uppsala, Sweden. http://www.vgscienta.com/productlist.aspx?MID=397

32 Omicron NanoTechnology GmbH, Taunusstein, Germany. http://www.omicron.de/en/products/ea-125-/instrument-concept

33 M.A. Kelly, M.L. Shek, P. Pianetta, T.M. Gür and M.R. Beasley, J. Vac. Sci. Technol. A, 2001, 19, 2127.

34 J. Pantförder, S. Pöllmann, J. F. Zhu, D. Borgmann, R. Denecke and H.-P. Steinrück, Rev. Sci. Instrum., 2005, 76, 014102.

35 F. Mangolini, J. Åhlund, G.E. Wabiszewski, V.P. Adiga, Egberts, F. Streller, K. Backlund, P.G. Karlsson, B. Wannberg and R.W. Carpick, Rev. Sci. Instrum., 2012, 83, 093112.

36 A. Jürgensen, N. Esser and R. Hergenröder, Surf. Interface Anal., 2012, 44, 1100.

37 H. Bluhm, M. Hävecker, A. Knop-Gericke, M. Kiskinova, R. Schlögl and M. Salmeron, MRS Bulletin, 2007, 32, 1022.

38 M. Salmeron and R. Schlögl, Surf. Sci. Rep., 2008, 63, 169.

39 A. Knop-Gericke, E. Kleimenov, M. Hävecker, R. Blume, D. Teschner, S. Zafeiratos, R. Schlögl, V.I. Bukhtiyarov, V.V. Kaichev, I.V. Prosvirin, A.I. Nizovskii, H. Bluhm, A. Barinov, P. Dudin and M. Kiskinova, High-pressure X-ray photoelectron spectroscopy: a tool to investigate heterogeneous catalytic processes, In: B.C. Gates, H. Knözinger, eds: Advances in Catalysis, Vol. 52, Burlington: Academic Press, 2009, pp. 213-272.

40 H. Bluhm, J. Electron Spectrosc. Relat. Phenom., 2010, 177, 71.

41 D.E. Starr, H. Bluhm, Z. Liu, A. Knop-Gericke and M. Hävecker, Application of Ambient Pressure X-ray Photoelectron Spectroscopy for the In-situ Investigation of Catalytically Relevant Processes, submitted, 2013.

A. Shavorskiy and H. Bluhm, Ambient pressure X-ray photoelectron spectroscopy, submitted to: "Handbook of Heterogeneous Catalysts for Clean Technology - Design, Analysis and Application", K. Wilson, A. Lee (Eds.), Wiley - VCH, Weinheim, Germany, 2013. J.A. Whaley, A.H. McDaniel, F. El Gabaly, R.L. Farrow, M.E. Grass, Z. Hussain, Z. Liu, M.A. Linne, H. Bluhm and K.F. McCarty, Rev. Sci. Instrum., 2010, 81, 086104.

R. Westerström, M.E. Messing, S. Blomberg, A. Hellman, H. Grönbeck, J. Gustafson, N.M. Martin, O. Balmes, R. van Rijn, J.N. Andersen, K. Deppert, H. Bluhm, Z. Liu, M.E. Grass, M. Hävecker and E. Lundgren, Phys. Rev. B, 2011, 83, 115440.

R. Toyoshima, M. Yoshida, Y. Monya, K. Suzuki, B.S. Mun, K. Amemeiya, K. Mase and H. Kondoh, J. Phys. Chem. Lett., 2012, 3, 3182.

46 S. Blomberg, M.J. Hoffmann, J. Gustafson, N.M. Martin, V.R. Fernandes, A. Borg, Z. Liu, R. Chang, S. Matera, K. Reuter and E. Lundgren, Phys. Rev. Lett., 2013, 110, 117601.

O. Balmes, A. Resta, D. Wermeille, R. Felici, M.E. Messing, K. Deppert, Z. Liu, M.E. Grass, H. Bluhm, R. Van Rjin, J.W.M. Frenken, R. Westerström, S.E.J. Blomberg, J.N. Andersen, J. Gustafson and E. Lundgren, Phys. Chem. Chem. Phys., 2012, 14, 4796. 
48 G. Ketteler, D.F. Ogletree, H. Bluhm, H. Liu, E.L.D. Hebenstreit and M. Salmeron, J. Am. Chem. Soc., 2005, 127, 18269.

49 D. Teschner, A. Pestryakov, E. Kleimenov, M. Hävecker, H. Bluhm, H. Sauer, A. Knop-Gericke and R. Schlögl, J. Catal., 2005, 230, 186.

50 D. Teschner, A. Pestryakov, E. Kleimenov, M. Hävecker, H. Bluhm, H. Sauer, A. Knop-Gericke and R. Schlögl, J. Catal., 2005, 230, 195.

51 J. Pantförder, S. Pöllmann, J. F. Zhu, D. Borgmann, R. Denecke and H.-P. Steinrück, Rev. Sci. Instrum., 2005, 76, 014102.

52 V.V. Kaichev, I.P. Prosvirin, V.I. Bukhtiyarov, H. Unterhalt, G. Rupprechter and H.-J. Freund, J. Phys. Chem. B, 2003, 107, 3522.

53 V.V. Kaichev, A.V. Miller, I.P. Prosvirin and V.I. Bukhtiyarov, Surf. Sci., 2012, 606, 420.

54 H. Gabasch E. Kleimenov, D. Teschner, S. Zafeiratos, M. Hävecker, A. Knop-Gericke, R. Schlögl, D. Zemlyanov, B. Aszalos-Kiss, K. Hayek and B. Klötzer, J. Catal., 2006, 242, 340.

D. Zemlyanov, B. Aszalos-Kiss, E. Kleimenov, D. Teschner, S. Zafeiratos, M. Hävecker, A. Knop-Gericke, R. Schlögl, H. Gabasch, W. Unterberger, K. Hayek and B. Klötzer, Surf. Sci., 2006, 600, 983.

H. Gabasch, W. Unterberger, K. Hayek, B. Klötzer, E. Kleimenov, D. Teschner, S. Zafeiratos, M. Hävecker, A. Knop-Gericke, R. Schlögl, J. Han, F.H. Ribeiro, B. Aszalos-Kiss, T. Curtin and D. Zemlyanov, Surf. Sci., 2006, 600, 2980.

D. Teschner, E. Vass, M. Hävecker, S. Zafeiratos, P. Schnörch, H. Sauer, A. Knop-Gericke, R. Schlögl, M. Chamam, A. Wootsch, A.S. Canning, J.J. Gamman, S.D. Jackson, J. McGregor and L.F. Gladden, J. Catal., 2006, 242, 26.

H. Gabasch, K. Hayek, B. Klötzer, W. Unterberger, E. Kleimenov, D. Teschner, S. Zafeiratos, M. Hävecker, A. Knop-Gericke, R. Schlögl, B. Aszalos-Kiss and D. Zemlyanov, J. Phys. Chem. C, 2007, 111, 7957.

R. Toyoshima, M. Yoshida, Y. Monya, Y. Kousa, K. Suzuki, H. Abe, B.S. Mun, K. Mase, K. Amemeiya and H. Kondoh, J. Phys. Chem. C, 2012, 116, 18691.

60 A. Haghofer, K. Föttinger, F. Girgsdies, D. Teschner, A. Knop-Gericke, R. Schlögl and G. Rupprechter, J. Catal., 2012, 286, 13.

61 C. Rameshan, C. Weilach, W. Stadlmayr, S. Penner, H. Lorenz, M. Hävecker, R. Blume, T. Rocha, D. Teschner, A. Knop-Gericke, R. Schlögl, D. Zemlyanov, N. Memmel, G. Rupprechter and B. Klötzer, J. Catal., 2010, 276, 113.

62 D. Teschner, J. Borsodi, Z. Kis, L. Szentmiklósi, Z. Révay, A. Knop-Gericke, R. Schlögl, D. Torres and Ph. Sautet, J. Phys. Chem. C, 2010, 114, 2293.

63 D. Teschner, Z. Révay, J. Borsodi, M. Hävecker, A. Knop-Gericke, R. Schlögl, D. Milroy, S.D. Jackson, D. Torres and Ph. Sautet, Angew. Chem. Int. Ed., 2008, 47, 9274.

E.M. Vass, M. Hävecker, S. Zafeiratos, D. Teschner, A. Knop-Gericke and R. Schlögl, J. Phys.: Condens. Matter, 2008, 20, 184016.

65 D. Teschner, J. Borsodi, A. Wootsch, Z. Révay, M. Hävecker, A. Knop-Gericke, S.D. Jackson and R. Schlögl, Science, 2008, 320, 86.

66 O. Pozdnyakova, D. Teschner, A. Wootsch, J. Kröhnert, B. Steinhauer, H. Sauer, L. Toth, F.C. Jentoft, A. Knop-Gericke, Z. Paál and R. Schlögl, J. Catal., 2006, 237, 17.

67 F. Tao, S. Dag, L.-W. Wang, Z. Liu, D.R. Butcher, H. Bluhm, M. Salmeron and G.A. Somorjai, Science, 2010, 327, 850. 
68 Z. Zhu, F. F. Tao, F. Zheng, R. Chang, Y. Li, L. Heinke, Z. Liu, M. Salmeron and G. A. Somorjai, Nano Letters, 2012, 12, 1491.

69 S. Günther, A. Scheibe, H. Bluhm, M. Hävecker, E. Kleimenov, A. Knop-Gericke, R. Schlögl and R. Imbihl, J. Phys. Chem. C, 2008, 112, 15382.

70 J. Y. Chung, F. Aksoy, M. E. Grass, H. Kondoh, P. Ross, Z. Liu and B. S. Mun, Surf. Sci., 2009, 603, L35.

71 D.R. Butcher, M.E. Grass, Z. Zeng, F. Aksoy, H. Bluhm, G.A. Somorjai, W.-X. Li, B.S. Mun and Z. Liu, J. Am. Chem. Soc., 2011, 133, 20319.

72 D. J. Miller, H. Öberg, S. Kaya, H. Sanchez Casalongue, D. Friebel, T. Anniyev, H. Ogasawara, H. Bluhm, L. G. M. Pettersson and A. Nilsson, Phys. Rev. Lett., 2011, 107, 195502.

73 R.B. Getman, W.F. Schneider, A.D. Smeltz, W.N. Delgass and F.H. Ribeiro, Phys. Rev. Lett., 2009, 102, 076101.

74 T. Shimada, B.S. Mun, I.F. Nakai, A. Banno, H. Abe, Y. Iwasawa, T. Ohta and H. Kondoh, J. Phys. Chem. C, 2010, 114, 17030.

75 Z. Zhu, D.R. Butcher, B. Mao, Z. Liu, M. Salmeron and G.A. Somorjai, J. Phys. Chem. C, 2013, 117, 2799.

76 A. Virnovskaia, S. Jørgensen, J. Hafizovic, Ø. Prytz, E. Kleimenov, M. Hävecker, H. Bluhm, A. Knop-Gericke, R. Schlögl and U. Olsbye, Surf. Sci., 2007, 601, 30.

77 L.R. Merte, J. Knudsen, F.M. Eichhorn, S. Porsgaard, H. Zeuthen, L.C. Grabow, E. Lægsgaard, H. Bluhm, M. Salmeron, M. Mavrikakis and F. Besenbacher, J. Am. Chem. Soc., 2011, 133, 10692.

78 D. Teschner, A. Wootsch, O. Pozdnyakova-Tellinger, J. Kröhnert, E.M. Vass, M. Hävecker, S. Zafeiratos, P. Schnörch, P.C. Jentoft, A. Knop-Gericke and R. Schlögl, J. Catal., 2007, 249, 318.

79 O. Pozdnyakova, D. Teschner, A. Wootsch, J. Kröhnert, B. Steinhauer, H. Sauer, L. Toth, F.C. Jentoft, A. Knop-Gericke, Z. Paál and R. Schlögl, J. Catal., 2006, 237, 1.

80 S. Porsgaard, L.R. Merte, L.K. Ono, F. Behafarid, J. Matos, S. Helveg, M. Salmeron, B. Roldan Cuenya and F. Besenbacher, ACS Nano, 2012, 6, 10743.

81 S. Schäfer, S.A. Wyrzgol, R. Caterino, A. Jentys, S.J. Schoell, M. Hävecker, A. Knop-Gericke, J.A. Lercher, I.D. Sharp and M. Stutzmann, J. Am. Chem. Soc., 2012, 134, 12528.

82 P. Jiang, S. Porsgaard, F. Borondics, M. Köber, A. Caballero, H. Bluhm, F. Besenbacher and M. Salmeron, J. Am. Chem. Soc., 2010, 132, 2858.

83 E.A. Willneff, S. Braun, D. Rosenthal, H. Bluhm, M. Hävecker, E. Kleimenov, A. Knop-Gericke, R. Schlögl and S.L.M. Schroeder, J. Am. Chem. Soc., 2006, 128, 12052.

84 T. Herranz, X. Y. Deng, A. Cabot, P. Alivisatos, Z. Liu, G. Soler-Illia and M. Salmeron, Cat. Today, 2009, 143, 158.

85 T. Herranz, X. Deng, A. Cabot, Z. Liu and M. Salmeron, J. Catal., 2011, 283, 119.

86 S. Porsgaard, P. Jiang, S. Borondics, S. Wendt, H. Bluhm, F.Besenbacher and M. Salmeron, Ang. Chem. Int. Ed., 2011, 50, 2266.

87 A.V. Bukhtiyarov, R.I. Kvon, A.V. Nartova, I.P. Prosvirin and V.I. Bukhtiyarov, Surf. Sci., 2012, 606, 559.

88 V.I. Bukhtiyarov, A.I. Nizovskii, H. Bluhm, M. Hävecker, E. Kleimenov, A. Knop-Gericke and R. Schlögl, J. Catal., 2006, 238, 260.

89 R.W. Joyner and M.W. Roberts, Chem. Phys. Lett., 1979, 60, 459. 
90 A.I. Boronin, V.I. Bukhtiyarov, A.L. Vishnevskii, G.K. Boreskov and V.I. Savchenko, Surf. Sci., 1988, 201, 195.

91 T.C.R. Rocha, A. Oestereich, D.V. Demidov, M. Hävecker, S. Zafeiratos, G. Weinberg, V.I. Bukhtiyarov, A. Knop-Gericke and R. Schlögl, Phys. Chem. Chem. Phys., 2012, 14, 4554.

92 D. V. Demidov, I. P. Prosvirin, A. M. Sorokin, T. Rocha, A. Knop-Gericke and V. I. Bukhtiyarov, Kin. Catal., 2011, 52, 855.

93 Y. Lei, F. Mehmood, S. Lee, J. Greeley, B. Lee, S. Seifert, R.E. Winans, J. W. Elam, R. J. Meyer, P.C. Redfern, D. Teschner, R. Schlögl, M.J. Pellin, L.A. Curtiss and S. Vajda, Science, 2010, 328, 224.

94 M.E. Glass, D. Butcher, Y. Zhang, Y. Li, H. Bluhm, K. Bratlie, T. Zhang, J.Y. Park and G.A. Somorjai, Angew. Chem. Int. Ed., 2008, 47, 8893.

95 F. Aksoy, M. E. Grass, S. H. Joo, N. Jabeen, Y. P. Hong, Z. Hussain, B. S. Mun and Z. Liu, Nucl. Instrum. Meth. A, 2011, 645, 260.

96 F.G. Requejo, E.L.D. Hebenstreit, D.F. Ogletree and M. Salmeron, J. Catal., 2004, 226, 83.

97 R.W. Joyner, M.W. Roberts, and S.P. Singh-Boparai, Surf. Sci. Lett., 1981, 104, L199.

98 R. Blume, M. Hävecker, S. Zafeiratos, D. Teschner, A. Knop-Gericke, R. Schlögl, P. Dudin, A. Barinov and M. Kiskinova, Cat. Today, 2007, 124, 71.

99 R. Blume, M. Hävecker, S. Zafeiratos, D. Teschner, E. Kleimenov, A. Knop-Gericke, R. Schlögl, A. Barinov, P. Dudin and M. Kiskinova, J. Catal., 2006, 239, 354.

100 D.E. Starr and H. Bluhm, Surf. Sci., 2013, 608, 241.

101 R. Blume, M. Hävecker, S. Zafeiratos, D. Teschner, E. Vass, P. Schnörch, A. Knop-Gericke, R. Schlögl, S. Lizzit, P. Dudin, A. Barinov and M. Kiskinova, Phys. Chem. Chem. Phys., 2007, 9, 3648.

102 K. Qadir, S.H. Joo, B.S. Mun, D.R. Butcher, J.R. Renzas, F. Aksoy, Z. Liu, G.A. Somorjai and J.Y. Park, Nano Letters, 2012, 12, 5761.

103 H.J. Ruppender, M. Grunze, C.W. Kong and M. Wilmers, Surf. Interface Anal., 1990, 15, 245.

104 B.C. Bayer, M. Fouquet, R. Blume, C.T. Wirth, R.S. Weatherup, K. Ogata, A. Knop-Gericke, R. Schlögl, S. Hofmann and J. Robertson, J. Phys. Chem. C, 2012, 116, 1107.

105 K. Wang, Z. Liu, T. H. Cruz, M. Salmeron and H. Liang, J. Phys. Chem. A, 2010, 114, 2489.

106 A. Caballero, J.P. Holgado, V.M. Gonzalez-delaCruz, S.E. Habas, T. Herranz and M. Salmeron, Chem. Comm., 2010, 46, 1097.

107 V.V. Kaichev, A.Yu. Gladky, I.P. Prosvirin, A.A. Saraev, M. Hävecker, A. Knop-Gericke, R. Schlögl and V.I. Bukhtiyarov, Surf. Sci., 2013, 609, 113.

108 V. Papaefthimiou, T. Dintzer, V. Dupuis, A. Tamion, F. Tournus, A. Hillion, D. Teschner, M. Hävecker, A. Knop-Gericke, R. Schlögl and S. Zafeiratos, ACS Nano, 2011, 5, 2182.

109 S. Zafeiratos, T. Dintzer, D. Teschner, R. Blume, M. Hävecker, A. Knop-Gericke and R. Schlögl, J. Catal., 2010, 269, 309.

110 S. Alayoglu, S.K. Beaumont, F. Zheng, V.V. Pushkarev, H. Zheng, V. Iablokov, Z. Liu, J. Guo, N. Kruse and G.A. Somorjai, Top. Catal., 2011, 54, 778.

111 X. Deng, A. Verdaguer, T. Herranz, Ch.D. Weiss, H. Bluhm, M. Salmeron, Langmuir 24, 9474 (2008).

112 D.M. Littrell and B.J. Tatarchuk, J. Vac. Sci. Techn. A, 1986, 4, 1608.

113 V.I. Bukhtiyarov, I.P. Prosvirin, E.P. Tikhomirov, V.V. Kaichev, A.M. Sorokin and V.V. Evstigneev, React. Kinet. Catal. Lett., 2003, 79, 181. 
114 K. Andersson, G. Ketteler, H. Bluhm, S. Yamamoto, H. Ogasawara, L.G.M. Pettersson, M. Salmeron and A. Nilsson, J. Am. Chem. Soc., 2008, 130, 2793.

115 K. Andersson, G. Ketteler, H. Bluhm, S. Yamamoto, H. Ogasawara, L.G.M. Pettersson, M. Salmeron and A. Nilsson, J. Phys. Chem. C, 2007, 111, 14493.

116 S. Günther, L. Zhou, M. Hävecker, A. Knop-Gericke, E. Kleimenov, R. Schlögl and R. Imbihl, J. Chem. Phys., 2006, 125, 114709.

117 S. Yamamoto, K. Andersson, H. Bluhm, G. Ketteler, D.E. Starr, Th. Schiros, H. Ogasawara, L.G.M. Pettersson and M. Salmeron. A. Nilsson, J. Phys. Chem. C, 2007, 111, 7848.

118 J.P. Baxter, M. Grunze and C.W. Kong, J. Vac. Sci. Technol. A, 1988, 6, 1123.

119 M. Behrens, F. Studt, I. Kasatkin, S. Kühl, Michael Hävecker, F. Abild-Pedersen, S. Zander, F. Girgsdies, P. Kurr, B.-L. Kniep, M.Tovar, R.W. Fischer, J.K. Nørskov and R. Schlögl, Science, 2012, 336, 893.

120 R. Würz, M. Rusu, Th. Schedel-Niedrig, M.Ch. Lux-Steiner, H. Bluhm, M. Hävecker, E. Kleimenov, A. Knop-Gericke and R. Schlögl, Surf. Sci., 2005, 580, 80.

121 M.A. Kelly, M.L. Shek, P. Pianetta, T.M. Gür and M.R. Beasley, J. Vac. Sci. Technol. A, 2001, 19, 2127.

122 Y. Enta, B. S. Mun, M. Rossi, J. P. N. Ross, Z. Hussain, C. S. Fadley, K.-S. Lee and S.-K. Kim, Appl. Phys. Lett., 2008, 92, 012110.

123 M. Rossi, B. S. Mun, Y. Enta, C. S. Fadley, K.-S. Lee, S.-K. Kim, H.-J. Shin, Z. Hussain and J. P. N. Ross, J. Appl. Phys., 2008, 103, 044104.

124 B.C. Bayer, C. Zhang, R. Blume, F. Yan, M. Fouquet, C. T. Wirth, R. S. Weatherup, L. Lin, C. Baehtz, R. A. Oliver, A. Knop-Gericke, R. Schlögl, S. Hofmann and J. Robertson, J. Appl. Phys., 2011, 109, 114314.

125 B.C. Bayer, S. Hofmann, C. Castellarin-Cudia, R. Blume, C. Baehtz, S. Esconjauregui, C. T. Wirth, R. A. Oliver, C. Ducati, A. Knop-Gericke, R. Schlögl, A. Goldoni, C. Cepek and J. Robertson, J. Phys. Chem., 2011, 115, 4359.

126 A. Rinaldi, J.-P. Tessonnier, M.E. Schuster, R. Blume, F. Girgsdies, Q. Zhang, T. Jacob, S.B. Abd Hamid, D.S. Su and R. Schlögl, Ang. Chem. Int. Ed., 2011, 50, 3313.

127 B. Frank, A. Rinaldi, R. Blume, R. Schlögl and D.S. Su, Chem. Mat., 2010, 22, 4462.

128 J. Zhang, X. Liu, R. Blume, A. Zhang, R. Schlögl and D.S. Su, Science, 2008, 322, 5898.

129 J. Zhang, D.S. Su, R. Blume, R. Schlögl, R. Wang, X. Yang and A. Gajović, Ang. Chem. Int. Ed., 2010, 49, 8640 .

130 St. Hofmann, R. Blume, Ch.T. Wirth, M. Cantoro, R. Sharma, C. Ducati, M. Hävecker, S. Zafeiratos, P. Schnörch, A. Oestereich, D. Teschner, M. Albrecht, A. Knop-Gericke, R. Schlögl and John Robertson, J. Phys. Chem. C, 2009, 113, 1648.

131 C. Zhang, F. Yan, B.C. Bayer, R. Blume, M.H. van der Veen, R. Xie, G. Zhong, B. Chen, A. Knop-Gericke, R. Schlögl, B.D. Capraro, S. Hofmann and J. Robertson, J. Appl. Phys., 2012, 111, 064310.

132 B. C. Bayer, S. Hofmann, C. Castellarin-Cudia, R. Blume, C. Baehtz, S. Esconjauregui, C.T. Wirth, R.A. Oliver, C. Ducati, A. Knop-Gericke, R. Schlögl, A. Goldoni, C. Cepek and J. Robertson, J. Phys. Chem. C, 2011, 115, 4359.

133 G. Ketteler, P. Ashby, B.S. Mun, I. Ratera, H. Bluhm, B. Kasemo and M. Salmeron, J. Phys.: Condens. Matt., 2008, 20, 184024.

134 A. Shavorskiy, F. Aksoy, M. Grass, Z. Liu, H. Bluhm and G. Held, J. Am. Chem. Soc., 2011, 133, 6659. 
135 A. Shavorskiy, T. Eralp, K. Schulte, H. Bluhm and G. Held, Surf. Sci., 2013, 607, 10.

136 G. Ketteler, S. Yamamoto, H. Bluhm, K. Andersson, D.E. Starr, D.F. Ogletree, H. Ogasawara, A. Nilsson and M. Salmeron, J. Phys. Chem. C, 2007, 111, 8278.

137 J. Haubrich, R. G. Quiller, L. Benz, Z. Liu and C. M. Friend, Langmuir, 2010, 26, 2445.

138 R. Jribi, E. Barthel, H. Bluhm, M. Grunze, P. Koelsch, D. Verreault and E. Sondergård, J. Phys. Chem. C, 2009, 113, 8273.

139 O. Rosseler, M. Sleiman, V. Nahuel Montesinos, A. Shavorskiy, V. Keller, N. Keller, M.I. Litter, H. Bluhm, M. Salmeron and H. Destaillats, J. Phys. Chem. Lett., 2013, 4, 536.

140 X. Deng, T. Herranz, Ch. Weis, H. Bluhm and M. Salmeron, J. Phys. Chem. C, 2008, 112, 9668.

141 J.T. Newberg, D.E. Starr, S. Porsgaard, S. Yamamoto, S. Kaya, E.R. Mysak, T. Kendelewicz, M. Salmeron, G.E. Brown, Jr., A. Nilsson and H. Bluhm, Surf. Sci., 2011, 605, 89.

142 J.T. Newberg, D.E. Starr, S. Porsgaard, S. Yamamoto, S. Kaya, E.R. Mysak, T. Kendelewicz, M. Salmeron, G.E. Brown, Jr., A. Nilsson and H. Bluhm, J. Phys. Chem. C, 2011, 115, 12864.

143 Y. Gassenbauer, R. Schafranek, A. Klein, S. Zafeiratos, M. Hävecker, A. Knop-Gericke and R. Schlögl, Phys. Rev. B, 2006, 73, 245312.

144 E. Kleimenov, H. Bluhm, M. Hävecker, A. Knop-Gericke, A. Pestryakov, D. Teschner, J. A. Lopez-Sanchez, J. K. Bartley, G.J. Hutchings and R. Schlögl, Surf. Sci., 2005, 575, 181.

145 M. Hävecker, R.W. Mayer, A. Knop-Gericke, H. Bluhm, E. Kleimenov, A. Liskowsi, D. Su, R. Follath, F. G. Requejo, D. F. Ogletree, M. Salmeron, J.A. Lopez-Sanchez, J. K. Bartley,G. J. Hutchings and R. Schlögl, J. Phys. Chem. B., 2003, 107, 4587.

146 M. Eichelbaum, M. Hävecker, Ch. Heine, A. Karpov, C.-K. Dobner, F. Rosowski, A. Trunschke and R. Schlögl, Angew. Chem. Int. Ed., 2012, 51, 6246.

147 E.J. Crumlin, E. Mutoro, Z. Liu, M.E. Grass, M.D. Biegalski, Y.-L. Lee, D. Morgan, H.M. Christen, H. Bluhm and Y. Shao-Horn, Energy Environ. Sci., 2012, 5, 6081.

148 J.L. Hueso, D. Martinez-Martinez, A. Caballero, A.R. Gonzalez-Elipe, B.S. Mun and M. Salmeron, Cat. Comm., 2009, 10, 1898.

149 M. Hävecker, S. Wrabetz, J. Kröhnert, L.-I. Csepei, R. Naumann d'Alnoncourt, Y.V. Kolen'ko, F. Girgsdies, R. Schlögl and A. Trunschke, J. Catal., 2012, 285, 48.

150 A.C. Sanfiz, T.W. Hansen, D. Teschner, P. Schnörch, F. Girgsdies, A. Trunschke, R. Schlögl, M.H. Looi and S.B. Abd Hamid, J. Phys. Chem. C, 2010, 114, 1912.

151 Y.-C. Lu, E.J. Crumlin, G.M. Veith, J.R. Harding, E. Mutoro, L. Baggetto, N.J. Dudney, Z. Liu and Y. Shao-Horn, Sci. Rep., 2012, 2, 715.

152 W.C. Chueh, A.H. McDaniel, M.E. Grass, Y. Hao, N. Jabeen, Z. Liu, S.M. Haile, K.F. McCarty, H. Bluhm and F. El Gabaly, Chem. Mat., 2012, 24, 1876.

153 C . Wen, Y. Zhu, Y. Ye, S. Zhang, F. Cheng, Y. Liu, P. Wang and F. Tao, ACS Nano, 2012, 6, 9305 .

154 S. Yamamoto, T. Kendelewicz, J.T. Newberg, G. Ketteler, D.E. Starr, E.R. Mysak, K. Andersson, H. Ogasawara, H. Bluhm, M. Salmeron, G.E. Brown, Jr. and A. Nilsson, J. Phys. Chem. C, 2010, 114, 2256.

155 E. de Smit, M.M. van Schooneveld, F. Cinquini, H. Bluhm, Ph. Sautet, F.M.F. de Groot and B.M. Weckhuysen, Angew. Chem. Int. Ed., 2011, 50, 1584.

156 X. Y. Deng, J. Lee, C. J. Wang, C. Matranga, F. Aksoy and Z. Liu, Langmuir, 2011, 27, 2146.

157 X. Y. Deng, J. Lee, C. J. Wang, C. Matranga, F. Aksoy and Z. Liu, J. Phys. Chem. C, 2010, 114, 22619. 
158 E. de Smit, F.M.F. de Groot, R. Blume, M. Hävecker, A. Knop-Gericke and B.M. Weckhuysen, Phys. Chem. Chem. Phys., 2010, 12, 667.

159 T. Kendelewicz, S. Kaya, J. T. Newberg, H. Bluhm, N. Mulakaluri, W. Moritz, M. Scheffler, A. Nilsson, R. Pentcheva and G. E. Brown, Jr., J. Phys. Chem. C, 2013, 117, 2719.

160 E.R. Mysak, D.E. Starr, K.R. Wilson and H. Bluhm, Rev. Sci. Instrum., 2010, 81, 016106.

161 A. Verdaguer, Ch.D. Weis, G. Oncins, G. Ketteler, H. Bluhm and M. Salmeron, Langmuir, 2007, 23, 9699.

162 A. Mura, I. Hedishemi, Z. Liu, T. Hosoi, H. Watanaba and K. Arima, J. Phys. Chem. C, 2013, 117, 165.

163 M. Krisch, R. D’Auria, M.A. Brown, D.J. Tobias, J.C. Hemminger, M. Ammann, D.E. Starr and H. Bluhm, J. Phys. Chem. C, 2007, 111, 13497.

164 M.A. Brown, Z. Liu, P.D. Ashby, A. Mehta, R.L. Grimm and J.C. Hemminger, J. Phys. Chem. C, 2008, 112, 18287.

165 M.A. Brown, P.D. Ashby, M.J. Krisch, Z. Liu, B.S. Mun, R.G. Green, J.B. Giorgi, J.C. Hemminger, J. Phys. Chem C, 2010, 114, 14093.

166 S. Ghosal, J.C. Hemminger, H. Bluhm, B.S. Mun, E.L.D. Hebenstreit, G. Ketteler, D.F. Ogletree, F.G. Requejo and M. Salmeron, Science, 2005, 307, 563.

167 K. Arima, P. Jiang, X. Deng, H. Bluhm and M. Salmeron, J. Phys. Chem. C, 2010, 114, 14900.

168 A. Krepelová, T. Huthwelker, H. Bluhm and M. Ammann, Chem. Phys. Chem., 2010, 11, 3859.

169 A. Verdaguer, J.J. Segura, J. Fraxedas, H. Bluhm and M. Salmeron, J. Phys. Chem. C, 2008, 112, 16898.

170 M.D. Baer, I-F.W. Kuo, H. Bluhm and S. Ghosal, J. Phys. Chem. B, 2009, 113, 15843.

171 S. Ghosal, M.A. Brown, M. Krisch, H. Bluhm, M. Salmeron, P. Jungwirth and J.C. Hemminger, J. Phys. Chem. A, 2008, 112, 12378.

172 S. Kaya, S. Yamamoto, J.T. Newberg, H. Bluhm, H. Ogasawara, T. Kendelewicz, G.E. Brown Jr., L. G. M. Pettersson and A. Nilsson, Sci. Rep., 2013, 3, 1074.

173 M.H. Cheng, K.M. Callahan, A.M. Margarella, D.J. Tobias, J.C. Hemminger, H. Bluhm and M.J. Krisch, J. Phys. Chem. C, 2012, 116, 4545.

174 H. Bluhm, D.F. Ogletree, Ch. Fadley, Z. Hussain, and M. Salmeron, J. Phys.: Condens. Matter, 2002, 4, L227.

175 A. Krepelová, J.T. Newberg, T. Huthwelker, H. Bluhm and M. Ammann, Phys. Chem. Chem. Phys., 2010, 12, 8870.

176 D.E. Starr, D. Pan, J.T. Newberg, M. Ammann, E.G. Wang, A. Michaelidis and H. Bluhm, Phys. Chem. Chem. Phys., 2011, 13, 19988.

177 A. Krepelová, Th. Bartels-Rausch, M.A. Brown, H. Bluhm and M. Ammann, J. Phys. Chem. A, 2013, 117, 401.

178 C. Zhang, M.E. Grass, A.H. McDaniel, S.C. DeCaluwe, F. El Gabali, Z. Liu, K.F. McCarty, R.L. Farrow, M.A. Linne, Z. Hussain, G.S. Jackson, H. Bluhm and B.W. Eichhorn, Nat. Mat., 2010, 9, 944.

179 S.C. DeCaluwe, M.E. Grass, C. Zhang, F. El Gabaly, H. Bluhm, Z. Liu, G.S. Jackson, A.H. McDaniel, K.F. McCarty, R.L. Farrow, M.A. Linne, Z. Hussain and B.W. Eichhorn, J. Phys. Chem. C, 2010, 114, 19853.

180 S.C. DeCaluwe, G.S. Jackson, R.L. Farrow, A.H. McDaniel, F. El Gabaly, K.F. McCarty, S. Nie, M.A. Linne, H. Bluhm, J.T. Newberg, Z. Liu and Z. Hussain, ECS Transactions, 2009, 16, 253. 
181 C. Zhang, M.E. Grass, Y. Yu, K.J. Gaskell, S.C. DeCaluwe, R. Chang, G.S. Jackson, Z. Hussain, H. Bluhm, B.W. Eichhorn and Z. Liu, ACS Catalysis, 2012, 2, 2297.

182 F. El Gabaly, A.H. McDaniel, M.E. Grass, Z. Liu, H. Bluhm, M.A. Linne, Z. Hussain, R.L. Farrow and K.F. McCarty, Phys. Chem. Chem. Phys., 2010, 12, 12138.

183 F. El Gabaly, A.H. McDaniel, M.E. Grass, W. Chueh, H. Bluhm, Z. Liu and K.F. McCarty, Chem. Comm., 2012, 48, 8338.

184 V. Papaefthimiou, M. Shishkin, D.K. Niakolas, M. Athanasiou, Y.T. Law, R. Arrigo, D. Teschner, M. Hävecker, A. Knop-Gericke, R. Schlögl, T. Ziegler, S.G. Neophytides and S. Zafeiratos, Adv. Energy Mat., 2013. DOI: 10.1002/aenm.201200727.

185 P.S. Miedema, M.M. van Schooneveld, R. Bogerd, T.C.R. Rocha, M. Hävecker, A. KnopGericke and F.M.F. de Groot, J. Phys. Chem. C, 2011, 115, 25422.

186 F. Tao, M. E. Grass, Y. W. Zhang, D. R. Butcher, J. R. Renzas, Z. Liu, J. Y. Chung, B. S. Mun, M. Salmeron and G. A. Somorjai, Science, 2008, 322, 932.

187 F. Tao, M. E. Grass, Y. W. Zhang, D. R. Butcher, F. Aksoy, S. Aloni, V. Altoe, S. Alayoglu, J. R. Renzas, C. K. Tsung, Z. W. Zhu, Z. Liu, M. Salmeron and G. A. Somorjai, J. Am. Chem. Soc, 2010, 132, 8697.

188 J.R. Renzas, W.Y. Huang, Y.W. Zhang, M.E. Grass, D.T. Hoang, S. Alayoglu, D.R. Butcher, F. Tao, Z. Liu and G.A. Somorjai, Phys. Chem. Chem. Phys., 2011, 13, 2556.

189 M.E. Grass, M. Park, F. Aksoy, Y. Zhang, M. Kunz, Z. Liu and B.S. Mun, Langmuir, 2010, 26, 16362.

190 S. Alayoglu, F. Tao, V. Altoe, C. Specht, Z.W. Zhu, F. Aksoy, D.R. Butcher, R.J. Renzas, Z. Liu and G.A. Somorjai, Cat. Lett., 2011, 141, 633.

191 F. Zheng, S. Alayoglu, V.V. Pushkarev, S.K. Beaumont, C. Specht, F. Aksoy, Z. Liu, J.H. Guo and G.A. Somorjai, Cat. Today, 2012, 182, 54.

192 M.D. Ong, B.W. Jacobs, J.D. Sugar, M.E. Grass, Z. Liu, G.M. Buffleben, W.M. Clift, M.E. Langham, P. Cappillino and D.B. Robinson, Chem. Mat., 2012, 24, 996.

193 D. Teschner, A. Wootsch and Z. Paál, Appl. Cat. A, 2012, 411, 31.

194 Y. Jugnet, D. Loffreda, C. Dupont, F. Delbecq, E. Ehret, F.J. Cadete Santos Aires, B.S. Mun, F. Aksoy Akgul and Z. Liu, J. Phys. Chem. Lett., 2012, 3, 3707.

195 Z. Paál, A. Wootsch, D. Teschner, K. Lázár, I.E. Sajó, N. Győrffy, G. Weinberg, A. KnopGericke and R. Schlögl, Appl. Cat. A, 2011, 391, 377.

196 C. Rameshan, W. Stadlmayr, S. Penner, H. Lorenz, N. Memmel, M. Hävecker, R. Blume, D. Teschner, T. Rocha, D. Zemlyanov, A. Knop-Gericke, R. Schlögl and B. Klötzer, Ang. Chem. Inter. Ed., 2012, 51, 3002.

197 M. Friedrich, D. Teschner, A. Knop-Gericke and M. Armbrüster, J. Catal., 2012, 285, 41.

198 V. Papaefthimiou, T. Dintzer, V. Dupuis, A. Tamion, F. Tournus, D. Teschner, M. Hävecker, A. Knop-Gericke, R. Schlögl and S. Zafeiratos, J. Phys. Chem. Lett., 2011, 2, 900.

199 V. Papaefthimiou, T. Dintzer, M. Lebedeva, D. Teschner, M. Hävecker, A. Knop-Gericke, R. Schlögl, V. Pierren-Bohnes, E. Savinova and S. Zafeiratos, J. Phys. Chem. C, 2012, 116, 14342.

200 L. Shao, W. Zhang, M. Armbrüster, D. Teschner, F. Girgsdies, B. Zhang, O. Timpe, M. Friedrich, R. Schlögl and D.S. Su, Ang. Chem. Int. Ed., 2011, 50, 10231.

201 K. Kovnir, M. Armbrüster, D. Teschner, T.V. Venkov, L. Szentmiklósi, F.C. Jentoft, A. KnopGericke, Yu. Grin and R. Schlögl, Surf. Sci., 2009, 603, 1784.

202 K. Kovnir, M. Armbrüster, D. Teschner, T.V. Venkov, F.C. Jentoft, A. Knop-Gericke, Y. Grin and R. Schlögl, Sci. Technol. Adv. Mater., 2007, 8, 420. 
203 Ch. Rameshan, W. Stadlmayr, S. Penner, H. Lorenz, L. Mayr, M. Hävecker, R. Blume, T. Rocha, D. Teschner, A. Knop-Gericke, R. Schlögl, D. Zemlyanov, N. Memmel and B. Klötzer, J. Catal., 2012, 290, 126.

204 B. Bridier, J. Pérez-Ramírez, A. Knop-Gericke, R. Schlögl and D. Teschner, Chem. Sci., 2011, 2, 1379.

205 S. Zafeiratos, F. Paloukis, G. Papakonstantinou, D. Teschner, M. Hävecker, E. Vass, P. Schnörch, A. Knop-Gericke, R. Schlögl, B. Moreno, E. Chinarro, J.R. Jurado and S.G. Neophytides, Cat. Today, 2010, 157, 250.

206 B. Halevi, E.J. Peterson, A. Delariva, E. Jeroro, V.M. Lebarbier, Y. Wang, J.M. Vohs, B. Kiefer, E. Kunkes, M. Hävecker, M. Behrens, R. Schlögl and A.K. Datye, J. Phys. Chem. C, 2010, 114, 17181.

207 B. Halevi, E.J. Peterson, A. Roy, A. DeLariva, E. Jeroro, F. Gao, Y. Wang, J.M. Vohs, B. Kiefer, E. Kunkes, M. Hävecker, M. Behrens, R. Schlögl and A.K. Datye, J. Catal., 2012, 291, 44.

208 C. Rameshan, W. Stadlmayr, C. Weilach, S. Penner, H. Lorenz, M. Hävecker, R. Blume, T. Rocha, D. Teschner, A. Knop-Gericke, R. Schlögl, N. Memmel, D. Zemlyanov, G. Rupprechter and B. Klötzer, Ang. Chem. Int. Ed., 2010, 49, 3224.

209 C. Rameshan, H. Lorenz, L. Mayr, S. Penner, D. Zemlyanov, R. Arrigo, M. Hävecker, R. Blume, A. Knop-Gericke, R. Schlögl and B. Klötzer, J. Catal., 2012, 295, 186.

210 S. Piccinin, S. Zafeiratos, C. Stampfl, T.W. Hansen, M. Hävecker, D. Teschner, V.I. Bukhtiyarov, F. Girgsdies, A. Knop-Gericke, R. Schlögl and M. Scheffler, Phys. Rev. Lett., 2010, 104, 035503.

211 F. Zheng, S. Alayoglu, V.V. Pushkarev, S.K. Beaumont, C. Specht, F. Aksoy, Z. Liu, J. Guo and G.A. Somorjai, Cat. Today, 2012, 182, 54.

212 G. Abrasonis, S. Wintz, M.O. Liedke, F. Aksoy, M. Krause, K. Kuepper, D. Banerjee, Z. Liu and S. Gemming, J. Phys. Chem. C, 2012, 116, 14401.

213 A. Toghan, R. Arrigo, A. Knop-Gericke and R. Imbihl, J. Catal., 2012, 296, 99.

214 M. Friedrich, D. Teschner, A. Knop-Gericke and M. Armbrüster, J. Phys. Chem. C, 2012, 116, 14930.

215 Y. Zhu, S. Zhang, Y. Ye, X. Zhang, L. Wang, W. Zhu, F. Cheng and F. Tao, ACS Catal., 2012, 2, 2403 (2012).

216 H. Pfnür and D. Menzel, J. Chem. Phys., 1983, 79, 2400.

217 J.C. Fuggle, T.E. Madey, M. Steinkilberg and D. Menzel, Surf. Sci., 1975, 52, 521.

218 F.M. Hoffmann J. Chem. Phys., 1989, 90, 2816.

219 F.M. Hoffmann and J.L. Robbins, J. Electron Spectrosc. Relat. Phenom., 1987, 45, 421.

220 N. Schumacher, A. Boisen, S. Dahl, A.A. Gokhale, S. Kandoi, L.C. Grabow, J.A. Dumesic, M. Mavrikakis and I. Chorkendorff, J. Catal., 2005, 229, 265.

221 J. Yoshihara and C.T. Campbell, J. Catal., 1996, 161, 776.

222 K. Andersson, A. Gómez, C. Glover, D. Nordlund, H. Öström, T. Schiros, O. Takahashi, H. Ogasawara, L. G. M. Pettersson and A. Nilsson, Surf. Sci., 2005, 585, L183.

223 T. Yamada, S. Tamamori, H. Okuyama and T. Aruga, Phys. Rev. Lett., 2006, 96, 036105.

224 T. Schiros, S. Haq, H. Ogasawara, O. Takahashi, H. Öström, K. Andersson, L.G.M. Pettersson, A. Hodgson and A. Nilsson, Chem. Phys. Lett., 2006, 429, 415.

225 J. Ren and S. Meng, J. Am. Chem. Soc., 2006, 128, 9282.

226 J. Nakamura, J.M. Campbell and C.T. Campbell, J. Chem. Soc. Faraday Trans., 1990, 86, 2725.

227 K. Bange, D. E. Grider, T. E. Madey and J. K. Sass, Surf. Sci., 1984, 136, 38. 
228 A. Spitzer and H. Lüth, Surf. Sci., 1982, 120, 376.

229 A. Spitzer and H. Lüth, Surf. Sci., 1985, 160, 353.

230 J. Kubota, J. Kondo, K. Domen and C. Hirose, Surf. Sci., 1993, 295, 169.

231 M. Polak, Surf. Sci., 1994, 321, 249.

232 D. Kolovos-Vellianitis and J. Küppers, J. Phys. Chem. B, 2003, 107, 2559.

233 Ch. Ammon, A. Bayer, H.P. Steinrück and G. Held, Chem. Phys. Lett., 2003, 377, 163.

234 W.D. Clendening, J.A. Rodriguez, J.M. Campbell, C.T. Campbell, Surf. Sci., 1989, 216, 429.

235 S. Hagstrom, H.B. Lyon and G.A. Somorjai, Phys. Rev. Lett., 1965, 15, 491.

236 L.L. Kesmodel, L.H. Dubois and G.A. Somorjai, Chem. Phys. Lett., 1987, 56, 267.

237 O. Björnehom, A. Nilsson, H. Tillborg, P. Bennich, A. Sandell, B. Hernnäs, C. Puglia, N. Mårtensson, Surf. Sci., 1994, 315, L983.

238 B.L.M. Hendriksen and J.W.M. Frenken, Phys. Rev. Lett., 2002, 89, 046101.

239 B.K Hodnett, Heterogeneous catalytic oxidation reaction: Fundamental and technological aspects of the selective and total oxidation of organic compounds, John Wiley \& Sons LTD, 2000.

240 Handbook of Heterogeneous Catalysis, Eds. G. Ertl, H. Knözinger, F. Schüth, J. Weitkamp, Wiley-VCH Verlag GmbH \& Co KGaA, Weinheim, Germany, 2008.

241 J. Schnadt, J. Knudsen, X.L. Hu, A. Michaelides, R.T. Vang, K. Reuter, Z. Li, E. Laegsgaard, M. Scheffler and F. Besenbacher, Phys. Rev. B, 2009, 80, 075424.

242 M. Rocca, L. Savio, L. Vattuone, U. Burghaus, V. Palomba, N. Novelli, F. Buatier de Mongeot, U. Valbusa, R. Gunnella, G. Comelli, A. Baraldi, S. Lizzit and G. Paolucci,,, 2000, 61, 213.

243 X. Bao, M. Muhler, T. Schedel-Niedrig, R. Schlögl, Phys. Rev. B 54, 2249 (1996).

244 D.N. Futuba, K. Hata, T. Yamada, T. Hiraoka, Y. Hayamizu, Y. Kakudate, O. Tanaike, M. Yumura and S. Iijima, Nat. Mater., 2006, 5, 987.

245 K.B.K. Teo, E. Minoux, L. Hudanski, F. Peauger, J.-P. Schell, P. Legagneux, D. Dieumegardt, G.A. Amaratunga and W.I. Milne, Nature, 2005, 437, 968.

246 F. Kreupl, A.P. Graham, G.S. Duesberg, W. Steinhoegl, M. Liebau, E. Unger and W. Hoenlein, Microelectron. Eng., 2002, 64, 399.

247 K. Hata, D.N. Futaba, K. Mizuno, T. Namai, M. Yumura and S. Iijima, Science, 2004, 306, 1362.

248 C. Mattevi, C.T. Wirth, S. Hofmann, R. Blume, M. Cantoro, C. Ducati, C. Cepek, A. KnopGericke, S. Milne, C. Castellarin-Cudia, S. Dolafi, A. Goldoni, R. Schlögl and J.J. Robertson, Phys. Chem. C, 2008, 112, 12207.

249 T. De los Arcos, F. Vonau, M.G. Garnier, V. Thommen, H.-G. Boyen, P. Oelhafen, M. Dueggelin, D. Mathis and R. Guggenheim, Appl. Phys. Lett., 2002, 80, 2383.

250 E.G. Colgan, J.P. Gambino and Q.Z. Hong, Mater. Sci. Eng. R, 1996, 16, 43.

251 C. Zhang, F. Yan, C.S. Allen, B.C. Bayer, S. Hofmann, B.J. Hickey, D. Cott, G. Zhong and J.J. Robertson, J. Appl. Phys., 2010, 108, 024311.

252 S.B. Adler, Chem. Rev., 2004, 104, 4791.

253 K. Kobayashi, Nucl. Instrum. Meth. A, 2009, 601, 32.

254 G.E. Brown, Jr., V.E. Henrich, W.H. Casey, D.L. Clark, C. Eggleston, A. Felmy, D.W. Goodman, M. Grätzel, G. Maciel, M.I. McCarthy, K.H. Nealson, D.A. Sverjensky, M.F. Toney and J.M. Zachara, Chem. Rev., 1999, 99, 77.

255 D. Emfietzogloua and H. Nikjoob, Rad. Res., 2007, 167, 110. 
256 S. Matsuyama, H. Mimura, H. Yumoto, H. Hara, K. Yamamura, Y. Sano, K. Endo, Y. Mori, Y. Nishino, K. Tamasaku, M. Yabashi, T. Ishikawa and K. Yamauchi, Proc. SPIE 5918, LaserGenerated, Synchrotron, and Other Laboratory X-Ray and EUV Sources, Optics, and Applications II, p. 591804, 2005.

257 W. Chao, P. Fischer, T. Tyliszczak, S. Rekawa, E. Anderson and P. Naulleau, Opt. Express, 2012, 23, 9777. 


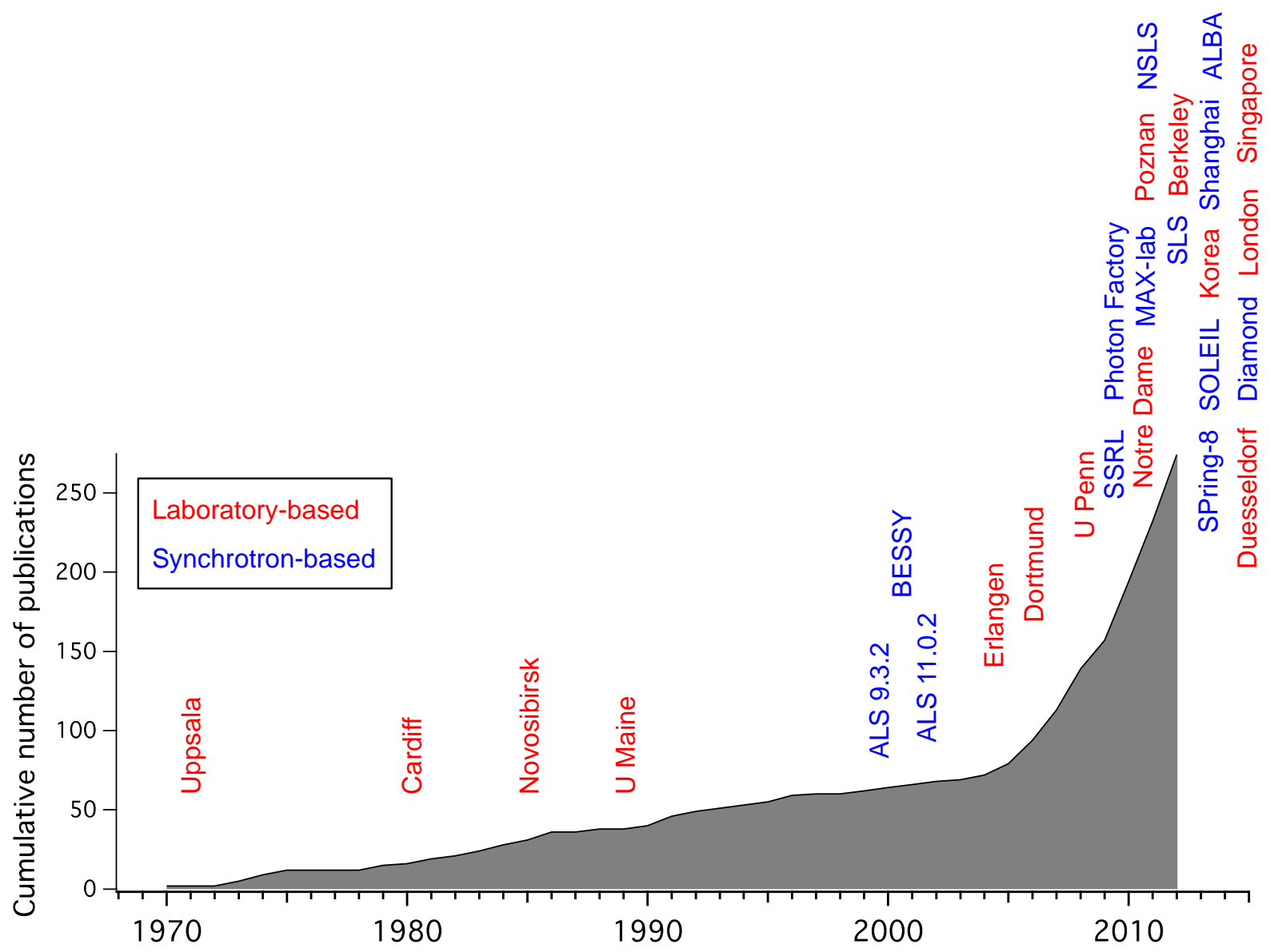

Figure 1

Ambient pressure XPS timeline, showing both the cumulative number of publications and the installation of new instruments. Red labels denote laboratory-based, blue labels synchrotron-based instruments. The dates for the installation of the instruments are approximate and to the best of our knowledge. 


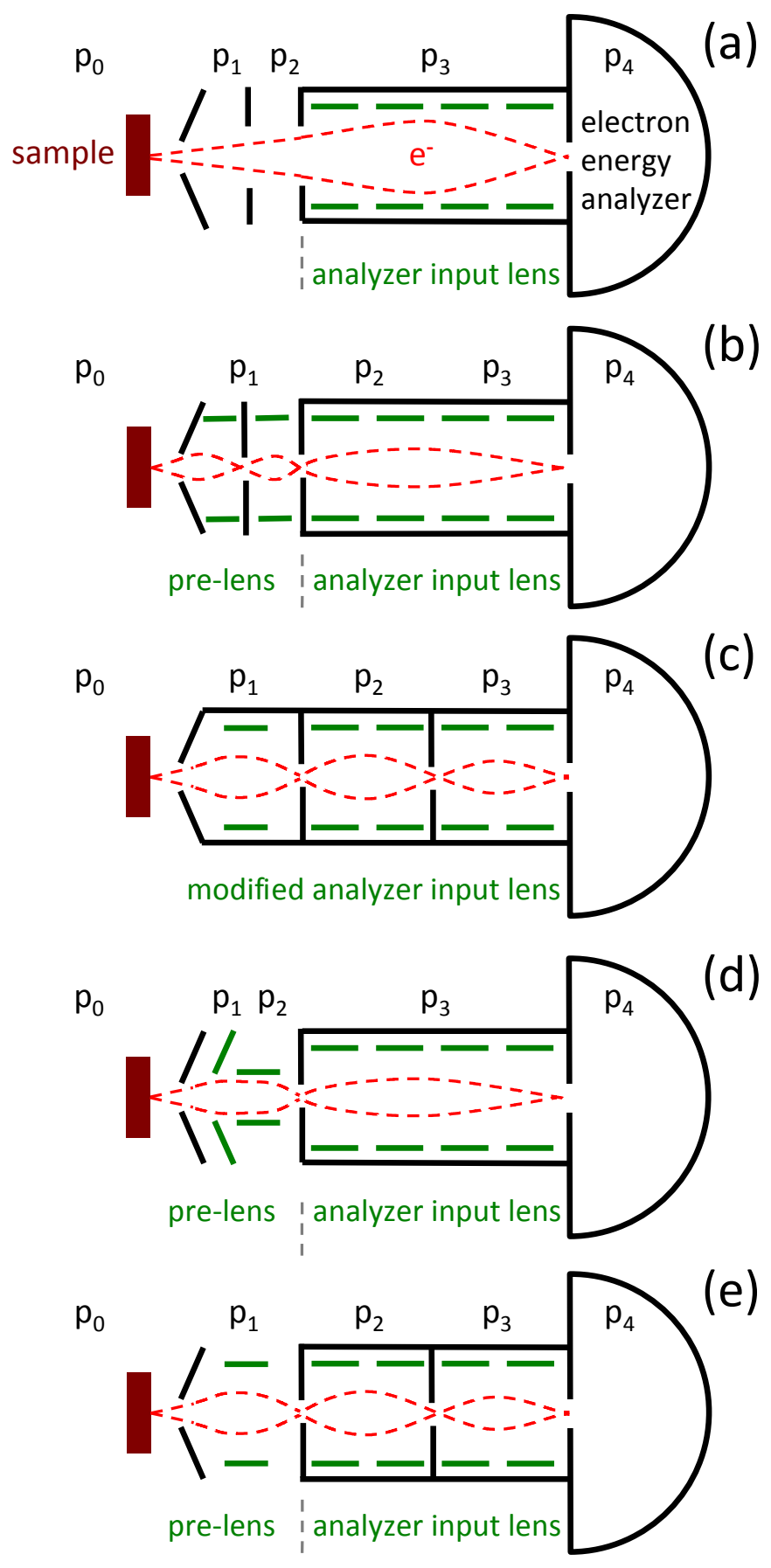

Figure 2

Principle layouts of differential pumping schemes for ambient pressure XPS. Schemes (b-e) use a variation of a differentially-pumped lens system, while scheme (a) uses a set of differentially-pumped apertures in front of a standard analyzer lens. For details see text. 

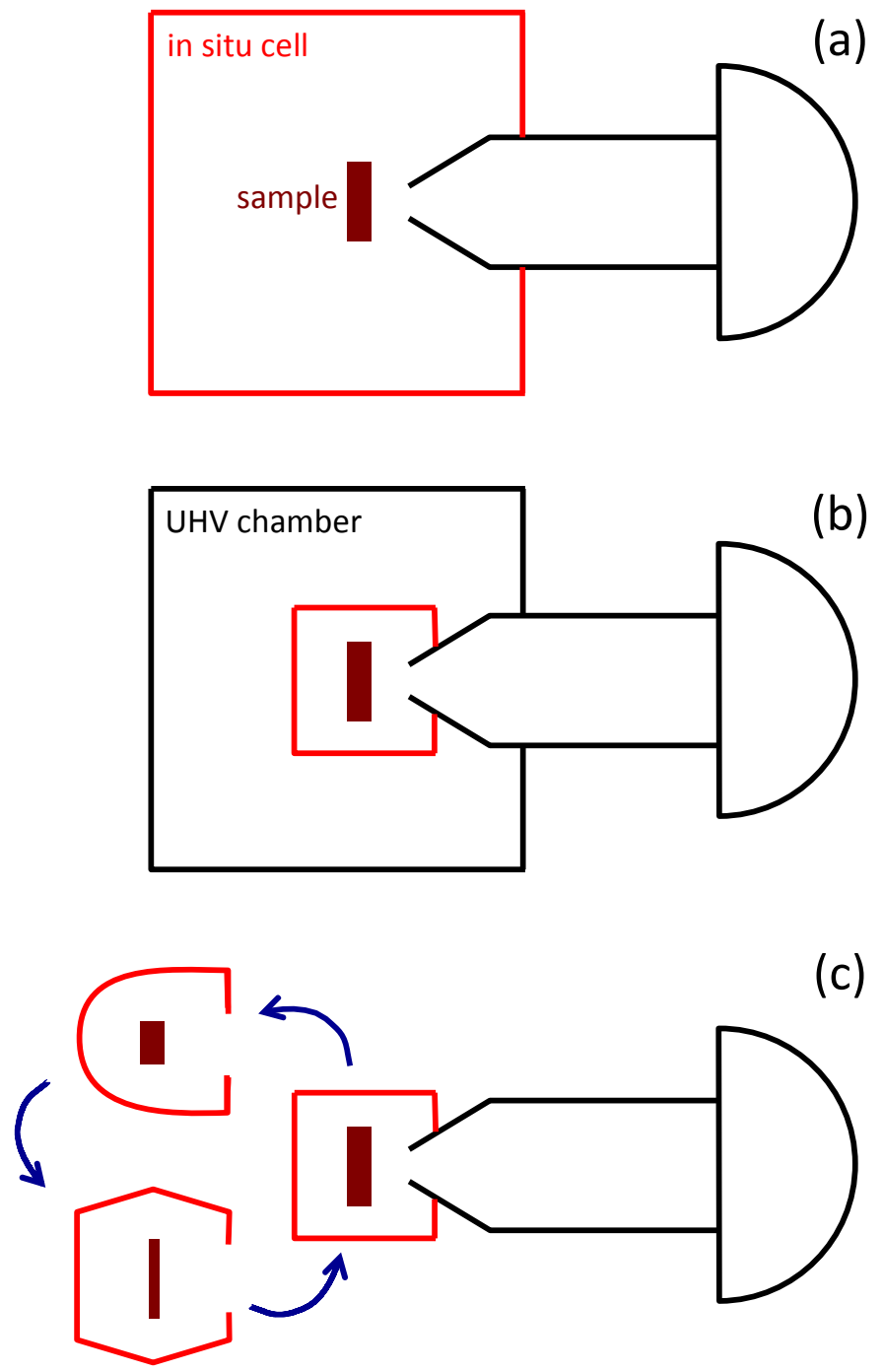

Figure 3

Principle layout of in situ measurement cells currently used in APXPS systems. (a) The analysis chamber/in situ cell is part of a vacuum system (often with load lock and preparation chamber). The whole chamber is exposed to gases during APXPS experiments. (b) The in situ cell is located inside a larger vacuum chamber. Only the in situ cell is exposed to gases during APXPS measurements. This enables to quickly switch between UHV-type and in situ experiments. (c) Exchangeable in situ cells, tailored to a particular experiment, are attached to the analyzer. This approach is best suited for complex or particularly "dirty" sample environments, such as in electrochemistry and investigations of liquid/vapor interfaces. 

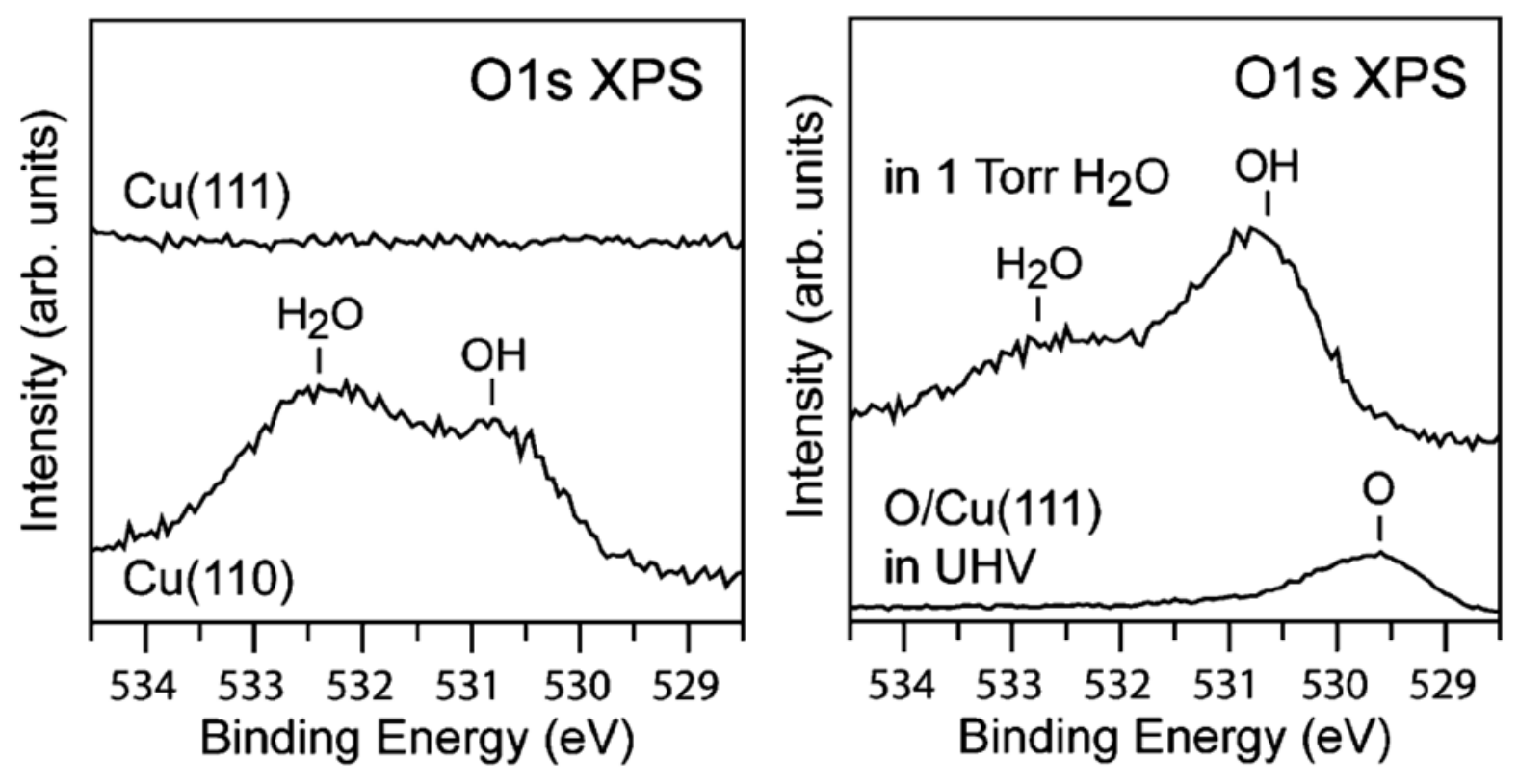

Figure 4

(left) O 1s spectra of $\mathrm{Cu}(110)$ and $\mathrm{Cu}(111)$ in 1.3 mbar of water at $298 \mathrm{~K}$. The spectra for the $\mathrm{Cu}(110)$ surface shows the presence of both $\mathrm{OH}$ and $\mathrm{H}_{2} \mathrm{O}$ while the $\mathrm{Cu}(111)$ surface shows the presence of neither $\mathrm{OH}$ or $\mathrm{H}_{2} \mathrm{O}$. (right) The adsorption of small amounts of $\mathrm{O}(0.12 \mathrm{ML})$ on $\mathrm{Cu}(111)$ causes the formation of $\mathrm{OH}$ groups and therefore $\mathrm{H}_{2} \mathrm{O}$ adsorption via hydrogen bonding at 1.3 mbar water and $298 \mathrm{~K}$. Reproduced with permission from Ref. [117]. 

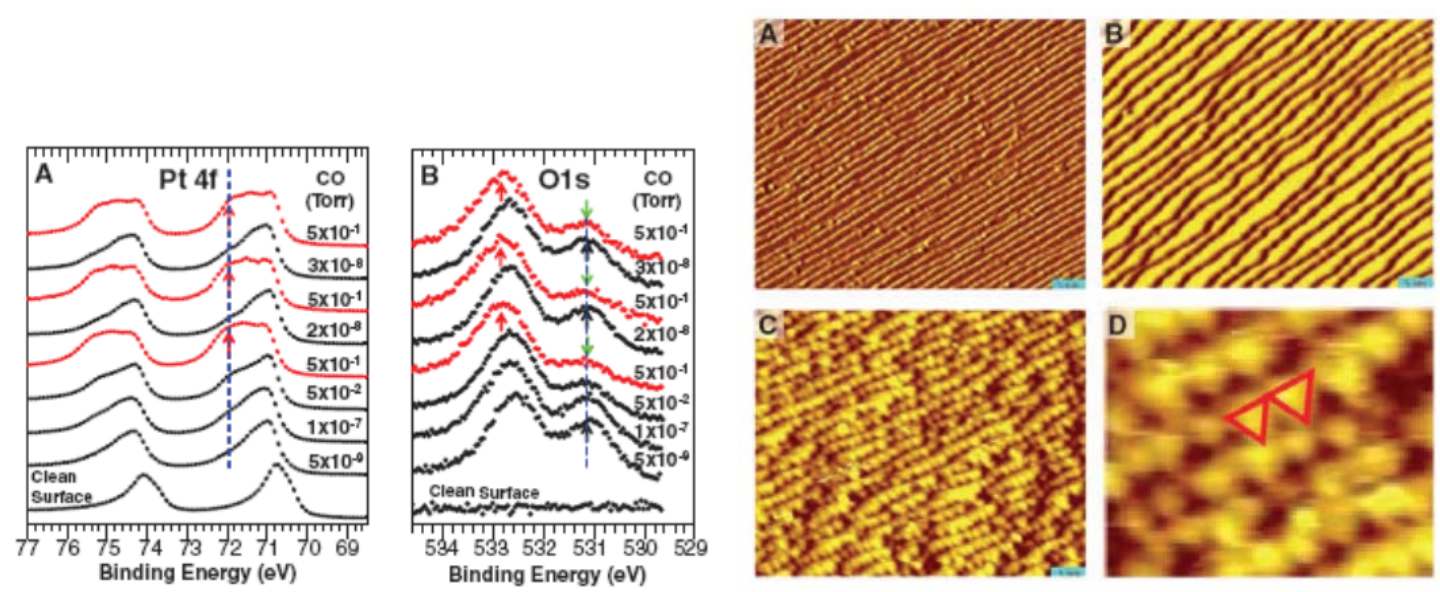

\section{Figure 5}

(left) $\mathrm{Pt} 4 \mathrm{f}$ and $\mathrm{O}$ 1s spectra for $\mathrm{CO}$ adsorption on $\mathrm{Pt}(557)$ at $\mathrm{CO}$ pressures indicated in the figure. The presence of high binding energy peaks at $72.15 \mathrm{eV}$ and $533.1 \mathrm{eV}$ in the $\mathrm{Pt} 4 \mathrm{f}$ and $\mathrm{O} 1 \mathrm{~s}$ spectra respectively are likely due to the adsorption of $\mathrm{CO}$ at under-coordinated Pt atoms on the stepped surface. (right) STM images at UHV conditions (A), $7 \times 10^{-8}$ mbar CO (B) and 1.3 mbar CO pressure (C and D) showing the formation of Pt nanoclusters at elevated pressure conditions. Reproduced with permission from Ref. [67]. 

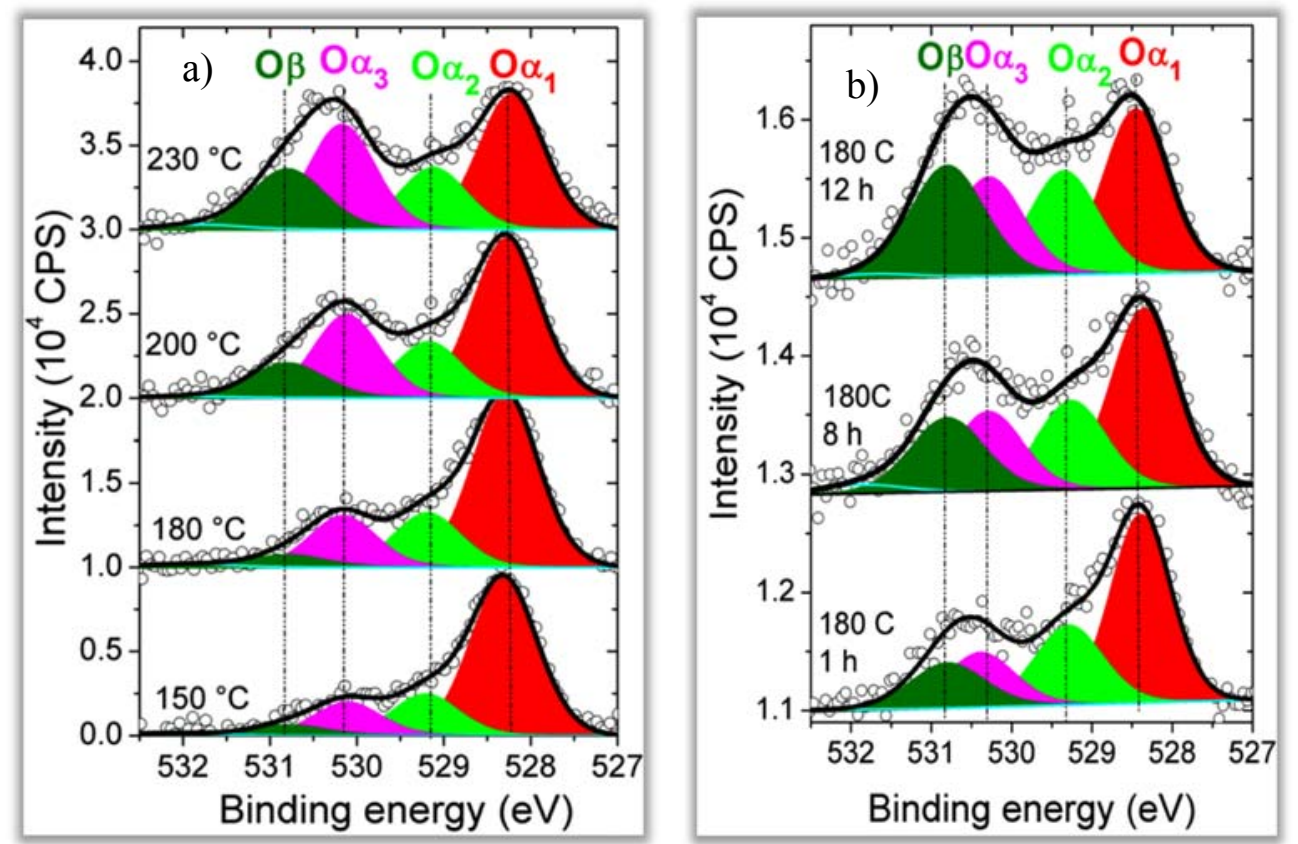

Figure 6

O 1s spectra following the formation kinetics of low temperature oxygen species on silver. Left: $\mathrm{Ag}(110)$ under $0.25 \mathrm{mbar}_{2}$ at different temperatures. Right: Silver powder with $45 \mu \mathrm{m}$ particle size at $180{ }^{\circ} \mathrm{C}$ after different reaction times with $\mathrm{O}_{2}$. Reproduced with permission from Ref. 88 . 

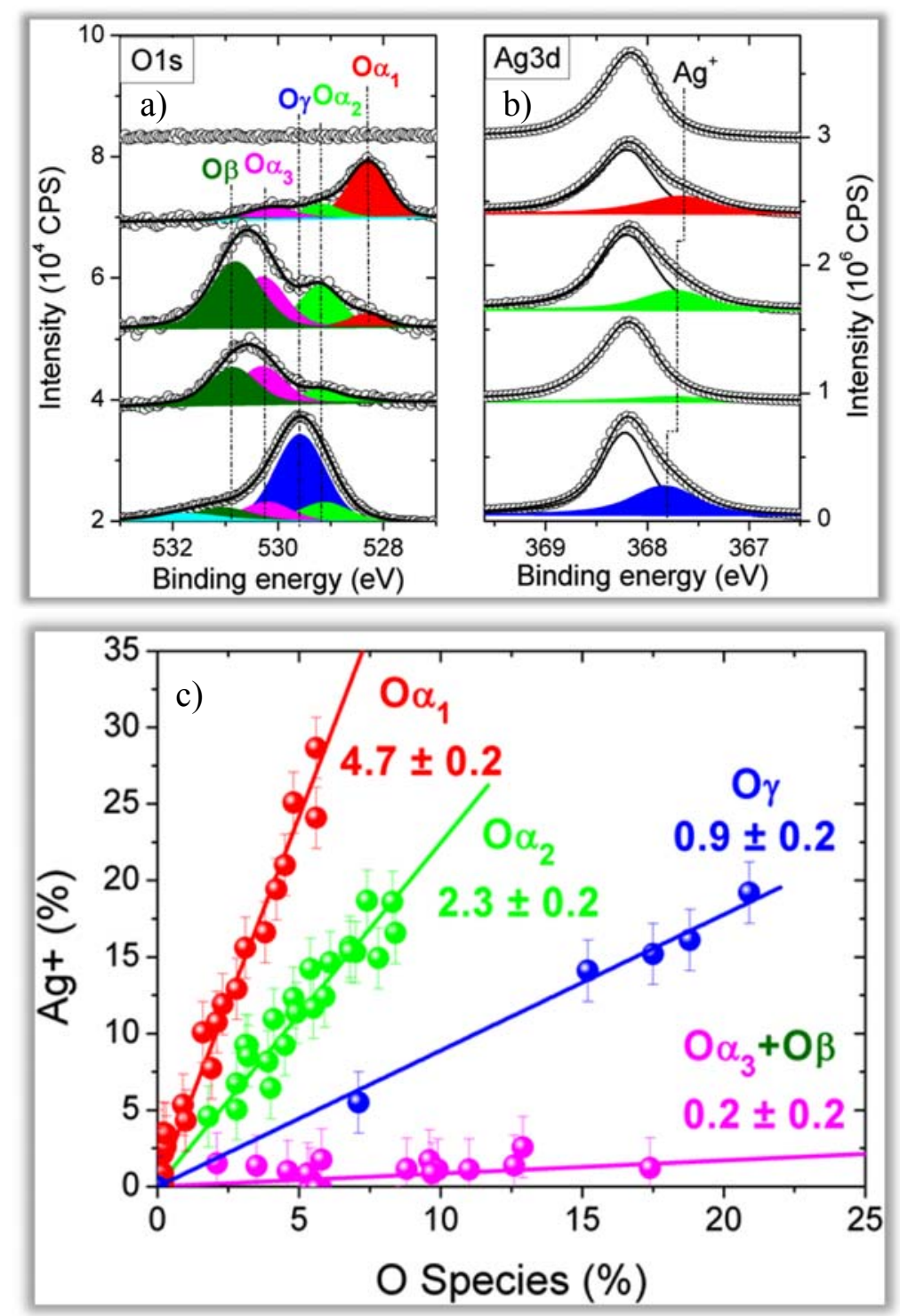

Figure 7

(top) $\mathrm{O} 1 \mathrm{~s}$ and $\mathrm{Ag} 3 \mathrm{~d}$ spectra showing the changes in the abundance of $\mathrm{Ag}^{+}$depending on the oxygen species present on the silver surface. (c) Quantitative correlation of the amount of $\mathrm{Ag}^{+}$ related to different oxygen species. Reproduced with permission from Ref. 88 . 


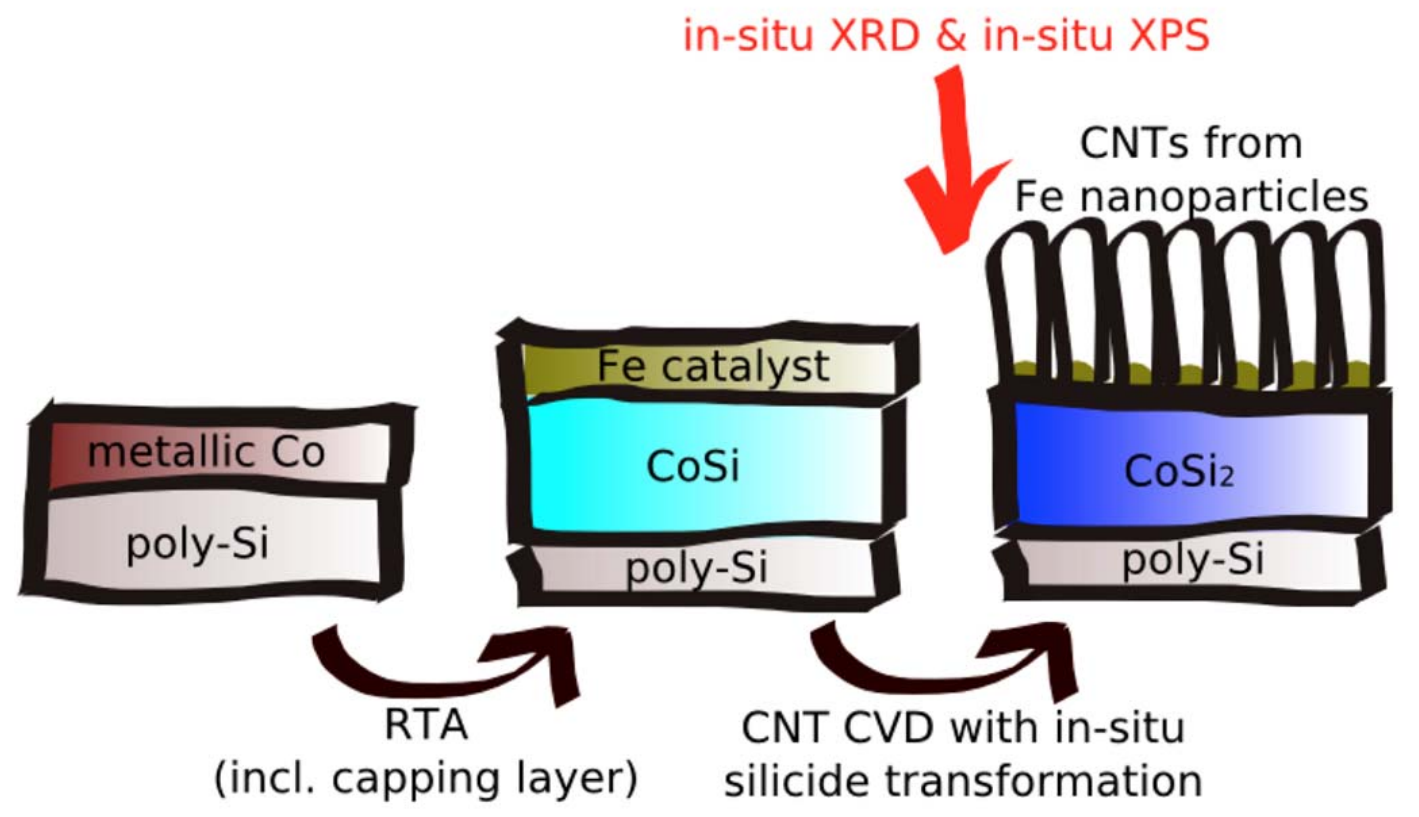

Figure 8

Schematic representation of the in situ preparation of CNT forests and the silicidation of high resistivity CoSi to low resistivity $\mathrm{CoSi}_{2}$ during CNT growth. Reproduced with permission from Ref. 124. 


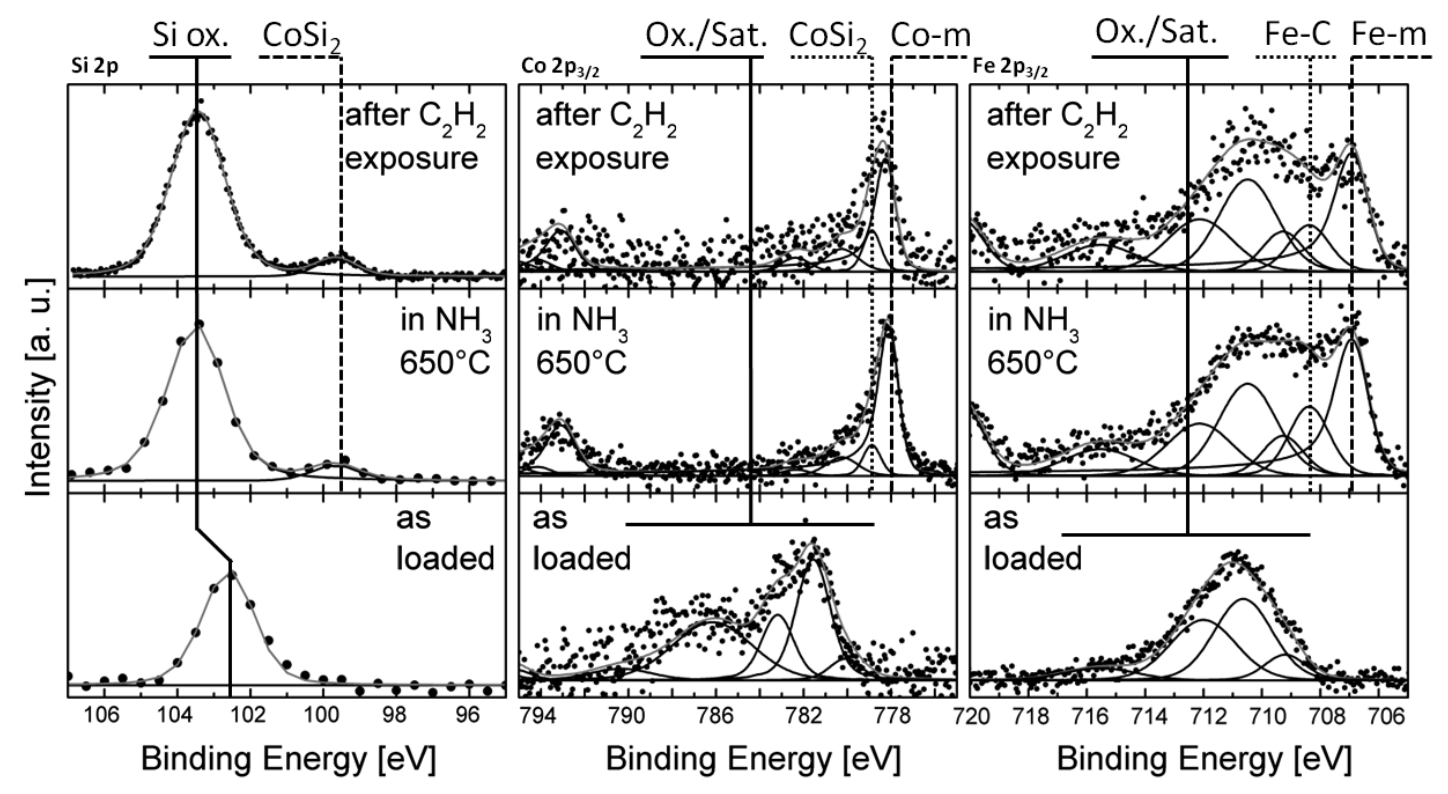

Figure 9

(from left to right) APXP spectra of the Si $2 \mathrm{p}, \mathrm{Co} 2 \mathrm{p}_{3 / 2}$ and $\mathrm{Fe} 2 \mathrm{p}_{3 / 2}$ regions showing the evolution of the surface $\left(\mathrm{Fe}, \mathrm{Co} ; \mathrm{E}_{\text {kin }}\left(\mathrm{e}^{-}\right)=150 \mathrm{eV}\right)$ and near surface $\left(\mathrm{Si} ; \mathrm{E}_{\text {kin }}\left(\mathrm{e}^{-}\right)=1000 \mathrm{eV}\right)$ chemistry under low pressure CVD. Reproduced with permission from Ref. 124. 
a

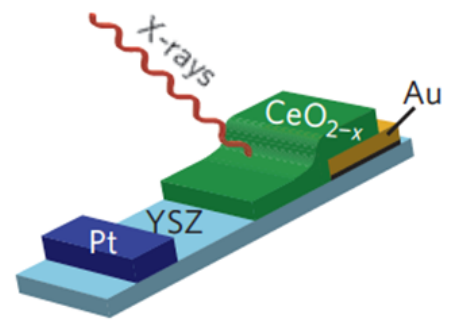

b

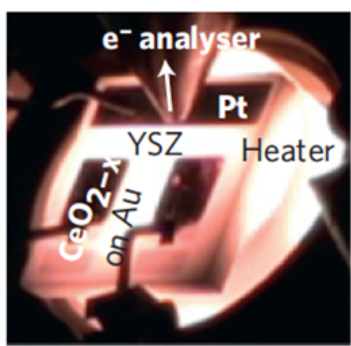

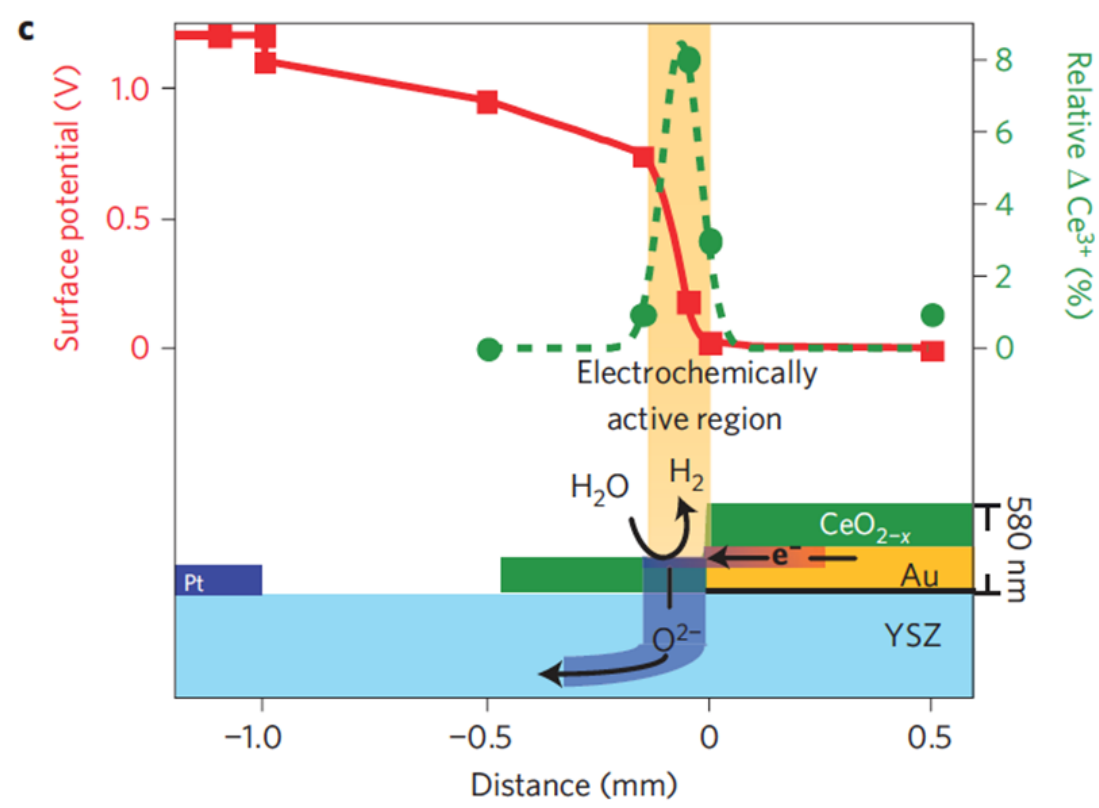

Figure 10

(a) Schematic layout of a solid oxide cells with a $200 \mathrm{~nm}$ thick Pt counter electrode, a $300 \mathrm{~nm}$ thick Au current collector on top of a $30 \mathrm{~nm}$ thick insulating alumina film (black), and a 50, 100 or $250 \mathrm{~nm}$ thick ceria working electrode patterned onto a polycrystalline YSZ substrate. This geometry exposes all cell components to the X-ray beam. The drawing is not to scale. (b) During operation, the cell is heated to $700{ }^{\circ} \mathrm{C}$ in the APXPS measurement position, close to the first aperture of the electrostatic lens system in a 1:1 gas mixture of $\mathrm{H}_{2}$ and $\mathrm{H}_{2} \mathrm{O}$ at a total pressure of about 1.3 mbar. (c) A 250-nm-thick ceria anode converts $\mathrm{H}_{2} \mathrm{O}$ to $\mathrm{H}_{2}$ and $\mathrm{O}^{2-}$ in a $150 \mu \mathrm{m}$ region at a cell potential of $+1.2 \mathrm{~V}$. APXPS reveals local surface potentials (red squares) and the relative change of the Ce oxidation state from equilibrium (green circles) in this region. Reproduced with permission from Ref. 178. 

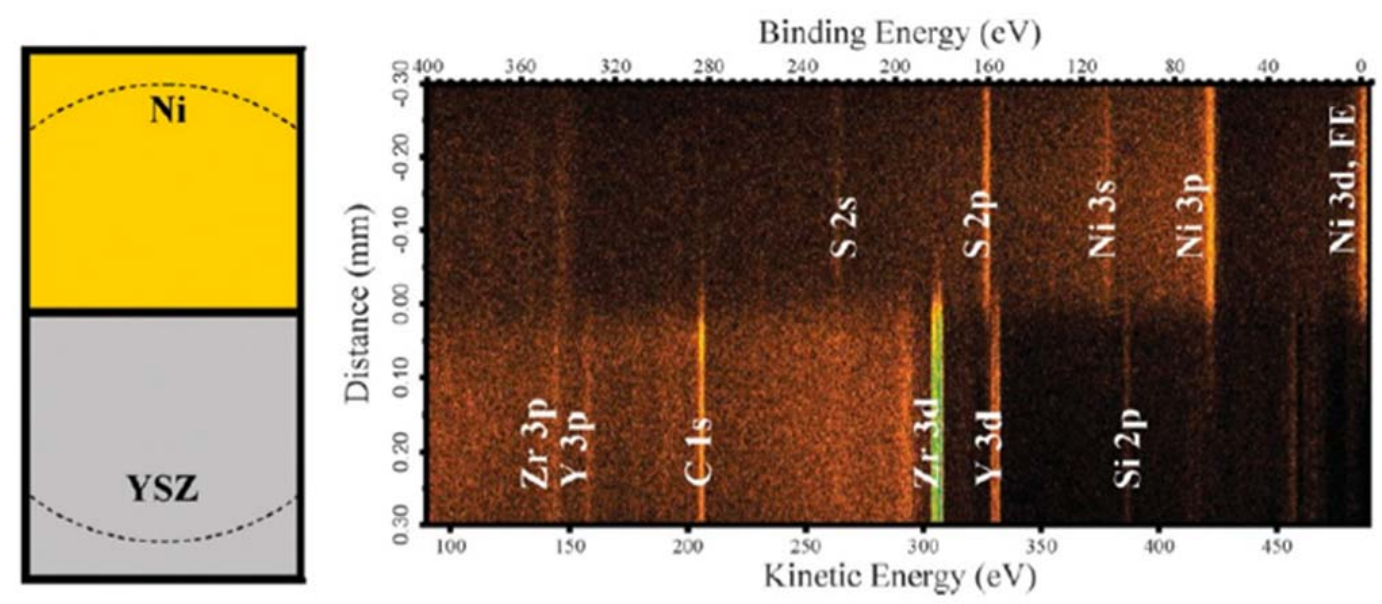

Figure 11

Left panel: top-view schematic of the SOEC Ni/YSZ interface measured in the APXPS image (right). The dashed lines shows the field-of-view (diameter $\sim 0.6 \mathrm{~mm}$ ). Right panel: The photoelectron binding energy versus real-space distance around the cell's three-phase boundary during operation at zero bias. Core level XPS peaks of Ni, YSZ, their impurities and the Ni Fermi edge (FE) are labeled. Intensities (counts) are displayed using a false-color scale. The binding energy scale is referenced to the Fermi edge of the grounded Ni electrode. Reproduced with permission from Ref. 182. 